\title{
One-Visibility Cops and Robber on Trees
}

\author{
A Thesis \\ Submitted to The Faculty of Graduate Studies and Research \\ in Partial Fulfillment of the Requirements \\ For the Degree of
}

\author{
Master of Science \\ in \\ Computer Science \\ University of Regina
}

By

Tanzina Akter

Regina, Saskatchewan

January, 2021

(C) Copyright 2021: Tanzina Akter 


\section{UNIVERSITY OF REGINA}

\section{FACULTY OF GRADUATE STUDIES AND RESEARCH SUPERVISORY AND EXAMINING COMMITTEE}

Tanzina Akter, candidate for the degree of Master of Science in Computer Science, has presented a thesis titled, One-Visibility Cops and Robber on Trees, in an oral examination held on December 14, 2020. The following committee members have found the thesis acceptable in form and content, and that the candidate demonstrated satisfactory knowledge of the subject material.

External Examiner:

Supervisor:

Committee Member:

Chair of Defense:

*via ZOOM Conferencing
*Dr. Yang Zhao, Department of Mathematics \& Statistics

${ }^{*}$ Dr. Boting Yang, Department of Computer Science

*Dr. Daryl Hepting, Department of Computer Science

*Dr. Shaun Fallat, Department of Mathematics \& Statistics 


\begin{abstract}
Graph searching comes into the limelight of research topics since it provides mathematical models for many real-world problems. Cops and robber is one aspect of graph searching that has recently gained much interest. This thesis provides results on a graph searching model, one-visibility cops and robber model.

The one-visibility cops and robber game is a variation of the zero-visibility cops and robber game, where one-visibility means that the robber is seen by a cop when the distance between them is at most one. Suppose that a robber is hiding on vertices of a graph. The focus of this thesis is finding the minimum number of one-visibility cops required to capture the robber. Such a number is called the one-visibility cop number. The literature does not provide enough results on the one-visibility cop number. In this thesis, we consider the one-visibility cops and robber game on trees. We prove a lower bound for the one-visibility cop number of trees. We propose strategies to clear trees according to their structures. We give a linear-time algorithm for computing the labels of all vertices of a tree. The first component in the label of the root is the cop number of that tree. We provide an optimal clearing guide for the cop player to clear a tree. We also present some interesting relations between zero- and one-visibility cop numbers on trees.
\end{abstract}




\section{Acknowledgements}

All thanks to the Almighty for giving me the strength and courage to complete this thesis.

My first debt of gratitude goes to my supervisor, Dr. Boting Yang for providing me an opportunity to work under his guidance as a research student. It would not be possible to complete my MSc thesis successfully without his constant support, encouragement, and invaluable guidance. I am highly grateful for his valuable suggestions and comments that help me to improve my presentation and writing skills.

I deeply acknowledge the financial support that I have received from the Faculty of Graduate Studies and Research (Scholarship, Graduate Teaching Assistantships) and the Department of Computer Science (Teaching Assistantships) which makes this work possible.

I especially thank Dr. Daryl Hepting for the time and effort he put in reading my thesis. His valuable suggestions and comments help me to improve my thesis.

I would love to extend my deepest gratitude to my husband, Md Monirul Islam for his continuous support throughout my graduate life. Last but not the least, I am highly thankful as well as highly grateful to my parents, Mosharaf Hossain and Jasmine Akter for their unconditional love and support throughout my entire life. 


\section{Contents}

Abstract i

\begin{tabular}{ll}
\hline Acknowledgements & i
\end{tabular}

List of Figures iv

$\begin{array}{lll}1 & \text { Introduction } & 1\end{array}$

$1.1 \quad$ Graph Searching Games . . . . . . . . . . . . . . . . . 2

1.2 Cops and Robber Game . . . . . . . . . . . . . . . 4

1.3 Main Contribution . . . . . . . . . . . . . . . 8

1.4 Organization $\ldots \ldots \ldots \ldots \ldots \ldots$

\begin{tabular}{lll}
\hline 2 & Preliminaries & 11
\end{tabular}

2.1 Definitions . . . . . . . . . . . . . . . . . . . . . . 11

2.2 One-Visibility Cops and Robber . . . . . . . . . . . . . 13 


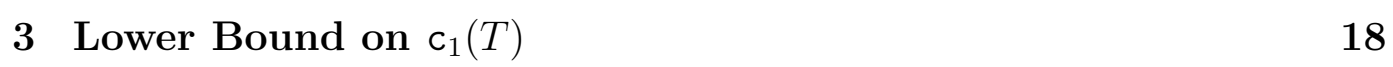

3.1 Lower Bound Theorem . . . . . . . . . . . . . . . . . . . . . 18

3.2 Cops' Strategies for Trees . . . . . . . . . . . . . . . . 24

4 One-Visibility Cop Number of a Tree 34

4.1 Notations and Definitions . . . . . . . . . . . . . 34

$4.2 \quad$ Algorithm for Computing $c_{1}(T)$. . . . . . . . . . . . . . 43

4.3 Correctness and Time Complexity . . . . . . . . . . . . . . . 56

4.4 An Optimal Clearing Guide of a Tree . . . . . . . . . . . . . . 64

$\begin{array}{lll}5 & \text { Relations between } c_{1}(T) \text { and } c_{0}(T) & 71\end{array}$

5.1 Comparing $c_{1}(T)$ and $c_{0}(T)$. . . . . . . . . . . . . 71

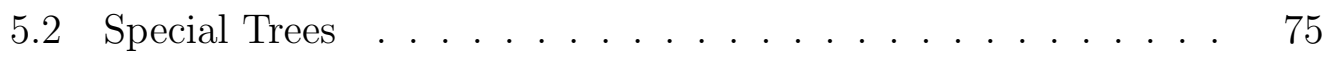

$5.2 .1 \quad$ Perfect Binary Trees . . . . . . . . . . . . . . . 75

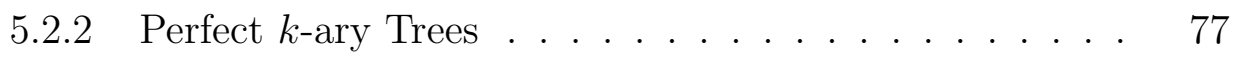

6 Conclusions and Future Work $\quad 79$

6.1 Summary $\ldots \ldots \ldots \ldots$. . . . . . . . . . . . . . . . . . . . . 79

6.2 Future Work . . . . . . . . . . . . . . . . . . . . . 80

\begin{tabular}{ll}
\hline References & 81
\end{tabular} 


\section{List of Figures}

$1.1 \quad$ A labeled 6 -cycle, $C_{6} \ldots \ldots \ldots \ldots$

$1.2 \quad$ A labeled cube $Q_{3} \quad \ldots \ldots \ldots \ldots$

$2.1 \quad c_{1}(T)=3$ and $c_{1}\left(T^{\prime}\right)=2 \ldots \ldots \ldots \ldots$

$3.1 \quad$ A tree $T$ with $\mathrm{c}_{1}(T)=2$ and $\mathrm{c}_{1}(H)=1$. . . . . . . . . . . 21

$3.2 \quad$ A tree $T$ with $\mathrm{c}_{1}(T)=3$ and $\mathrm{c}_{1}(H)=1$. . . . . . . . . . . 21

$3.3 \quad T_{1}$ consists of $X_{1}, X_{2}, X_{3}$ and the paths to $v$. . . . . . . . 24

$3.4 \quad$ A tree $T$ with $c_{1}(T)=k$, where $v$ is a hub. . . . . . . . . . 25

$3.5 \quad$ A tree $T$ with $\mathrm{c}_{1}(T)=k$, where $v$ is an avenue vertex. . . . . . 25

$3.6 \quad$ A tree $T$ with $c_{1}(T)=k$, where $u$ and $v$ are street vertices. . . 26

$3.7 \quad$ A tree $T$ with $c_{1}(T)=2$, where $x_{1}$ and $x_{2}$ are avenue vertices of $T$ and all others are not hubs, street vertices or avenue vertices of $T$. . . . . . . . . . . . . . . . . . . . 28

$3.8 \quad$ A tree $T$ with $c_{1}(T)=2$, in which every vertex is a hub. . . . 29 
4.1 Labels of vertices . . . . . . . . . . . . . . . . . . . . . 40

$4.2 \quad$ A tree $T^{[u]}$ with $\mathrm{c}_{1}\left(T^{[u]}\right)=k+1$, where $L_{T^{\left[v_{1}\right]}}\left(v_{1}\right)=\left(k, \perp, z_{1} \sim\right.$ $\left.z_{2} ; 1,1 ; 0,0\right)$ and $L_{T^{\left[v_{2}\right]}}\left(v_{2}\right)=\left(k, \perp, v_{2} ; 0,0 ; 0,1\right) . \ldots \ldots \ldots$

$4.3 \quad$ A tree $T^{[u]}$ with $\mathrm{c}_{1}\left(T^{[u]}\right)=k$, where $L_{T^{\left[v_{1}\right]}}\left(v_{1}\right)=\left(k, \perp, z_{1} \sim\right.$ $\left.z_{2} ; 1,1 ; 0,0\right)$ and $L_{T^{\left[v_{2}\right]}}\left(v_{2}\right)=\left(k, \perp, v_{2} ; 0,0 ; 0,0\right) . \ldots \ldots \ldots$

$4.4 \quad$ A tree $T^{[u]}$ with $c_{1}\left(T^{[u]}\right)=k$, where $v_{1}, v_{2}$ and $v_{3}$ are three $k$-pre-branching children of $u$. . . . . . . . . . . . 50

$4.5 \quad \mathrm{~A}$ tree $T^{[u]}$ with $\mathrm{c}_{1}\left(T^{[u]}\right)=k$, where $L_{T^{\left[v_{1}\right]}}\left(v_{1}\right)=\left(k, \perp, v_{1} ; 0,0 ; 1,0\right)$ and $L_{T^{\left[v_{2}\right]}}\left(v_{2}\right)=\left(k, \perp, v_{2} \sim z_{1} ; 0,0 ; 1,0\right)$ and $L_{T^{\left[v_{3}\right]}}\left(v_{3}\right)=(k, \perp$ ,$\left.v_{3} ; 0,0 ; 0,2\right) . \ldots \ldots \ldots \ldots \ldots \ldots \ldots \ldots \ldots \ldots \ldots \ldots$

$4.6 \quad$ A tree $T^{[u]}$ with $\mathrm{c}_{1}\left(T^{[u]}\right)=k$, where $u \sim z_{1}$ is the road containing an avenue vertex $y_{1} \ldots \ldots \ldots \ldots \ldots \ldots$

$4.7 \quad$ A tree $T^{[u]}$ with $\mathrm{c}_{1}\left(T^{[u]}\right)=k$, where $u \sim z_{1}$ is the road containing street vertices $z_{1}$ and $p_{1}$. . . . . . . . . . . . . 52

4.8 A tree $T^{[u]}$ with $c_{1}\left(T^{[u]}\right)=k$ and with no street or avenue vertex, the road $u \sim z_{1}$ is the road containing a hub $z_{1}$. . . . 52

$4.9 \quad$ A tree $T^{[u]}$ with $\mathrm{c}_{1}\left(T^{[u]}\right)=k$ and a hub $u$ in $T^{[u]} . \ldots . . \quad 53$

4.10 Computing the label of $u$ in $T^{[u]} . \ldots \ldots \ldots \ldots$

4.11 Tree $T_{i}^{\left[x_{i}^{u}\right]}, i \in\{2,3, \ldots, m\} \ldots \ldots \ldots \ldots \ldots$

$5.1 \quad$ A tree where each dashed line represents a path of length at least $8 . \ldots \ldots \ldots$. . . . . . . . . . . . . . . 74 
5.2 Tree $T_{5 m+5}^{2}$ with $\operatorname{root} u$. . . . . . . . . . . . . 76

5.3 Tree $T_{5 m+9}^{2}$ with $\operatorname{root} u$. . . . . . . . . . . . . . . . . 77

$5.4 \quad$ Tree $T_{4 m}^{3}$ with $\operatorname{root} u$. . . . . . . . . . . . . . . . . . . . 78 


\section{Chapter 1}

\section{Introduction}

Graph searching is closely related to real-world problems such as eliminating a computer virus in a network, computer games, and even counter-terrorism. Graph searching problems consider two notions cops and robber. In some research cops are referred to as searchers and robber is referred to as fugitive or intruder. The purpose of cops (searchers) is to capture the robber (fugitive). A natural question here is: What is the fewest number of cops (or searchers) required to capture the robber? This thesis is motivated by this question and also considers a few other constraints like visibility and actions of the cops. In literature, graph searching is referred by many names such as Cops and Robber game or pursuit-evasion game. The broad overview of many graph searching models are given in [3] and some aspects of cops and robber are described in [2]. Here in the first section, we give a brief overview of graph searching games and in the later section, we give a brief overview of cops and robber game. 


\subsection{Graph Searching Games}

In many graph searching problems, there are searchers trying to capture a fugitive (intruder) throughout the game. In searching games, the robber can hide in an edge or vertex and can move at high speed at any time (the movement depends on the model). When the robber moves at a great speed in any direction then the famous moniker searching is used. Graph searching has become an important topic and been used in contexts such as pebbling, VLSI circuit design, assuring privacy while using a bugged channel [3]. Graph searching games are linked with various parameters in literature such as treewidth, pathwidth, cutwidth. They can give deep insight into graph structures and can provide efficient algorithms. In the last three decades, a large number of searching games have been evolved.

The first searching model was introduced by Parsons [14], and used where continuous searching is required. There are many versions of Parsons model and the difference mainly relies on the movement of the robber such as i) the robber is invisible and active, and ii) the robber is visible or lazy. Edge searching, node searching, and mixed searching lie in the first category where there are restrictions on searchers but no restrictions on the robber. The edge searching game was introduced by Megiddo et al. [12]. The three actions performed by searchers in the edge searching are: placing a searcher on a vertex, removing a searcher from a vertex, and sliding a searcher from one vertex to another along an edge. In edge searching, the robber can hide in edges and searchers will traverse the edges to capture the robber. The robber 
will be captured when a searcher and the robber both occupy the same vertex. The goal of edge searching is to find the minimum number of searchers to clear a given graph. Yang [20] introduced the fast edge searching model, which uses the same setting as edge searching but the goal is to find the minimum number of steps to clear trees. The node searching game was introduced by Kirousis and Papadimitriou [11]. There are only two actions for searchers: placing a searcher on a vertex and removing a searcher from a vertex. In node searching, the robber is captured either when both searchers and robber occupy the same vertex or the robber is on an edge whose endpoints are both occupied by searchers. The mixed searching game was introduced by Bienstock and Seymour [1]. In mixed searching, the operations performed by the searchers are the same as edge searching but the conditions for capturing the robber are the combination of edge searching and node searching. Fast searching model was introduced by Dyer et al. 99]. The goal of fast searching is different than other searching models. In this model, the main focus is to clear the graph in a minimum number of steps. This model is important in cases where it is affordable to use more searchers instead of allowing a fugitive to be free. For example, it is always important to capture a dangerous hidden fugitive as early as possible regardless of the cost of using more policemen. The fast edge search game has close relation with fast search game. The difference between fast search and fast edge search is that in fast search every edge can be traversed exactly once by a searcher and searchers can not be removed from the graph. Another searching model is graph guarding introduced by Fomin et al. [10]. In this model, a region in the graph is protected by searchers. The 
robber will try to enter the protected region and if he can then it will be a robber win graph. The searchers have all the information about others and if they can protect the region from robber then it is a cop win graph. The goal of all major search problems is to find the minimum number of searchers. A brief survey about searching models is found in [3].

\subsection{Cops and Robber Game}

In recent years another aspect of graph searching "Cops and Robber" has received much attention. In this thesis, we will focus on the Cops and Robber game. Cops and Robber is a well-known pursuit-evasion game played on graphs. This game is played by two players: the first player controls a collection of agents, known as cops and the second player controls a single robber. The cops move through the graph attempting to capture the robber, while the robber moves to avoid the cops. In the cops and robber game, cops and robber occupy only vertices and they move alternatively to their neighbours. The basic actions performed by a cop player are, i) place: a cop is placed on a vertex, ii) move: a cop moves from one vertex to another along an edge, iii) pass: a cop stays on the same vertex. The game is played over a sequence of rounds on a graph. At round 0, cops are placed on a set of vertices of the graphs, and the robber is placed on a vertex of the graph. Cops move first to an adjacent vertex in round 1 and then the robber moves to an adjacent vertex. The robber is captured if a cop and the robber occupy the same vertex after some finite number of rounds. We define a graph as cop-win if the robber 
is captured by a cop. If this situation never happens then it is a robber-win graph. A cop win strategy is a set of rules followed by the cops which results in the capture of the robber. A robber win is a strategy where robber can evade the cops indefinitely. If we place a cop on each vertex then the robber will be captured in round 0 . So the minimum number of cops to capture a robber is a well defined positive integer and it is defined as cop number of the graph $G$. The cop number of a graph $G$ is denoted by $c(G)$. If $c(G)=k$ then $G$ is called a $k$-cop win graph. If $k=1$, then it is a special case and $G$ is simply called cop win graph.

An example is given here to understand how to play the game. Let us play the game on a 6 -cycle $C_{6}$. The vertices are labeled as 1,2,3,4,5,6 in Figure 1.1 . Suppose, a cop is placed on vertex 1 . If the robber chooses vertex 1 then it

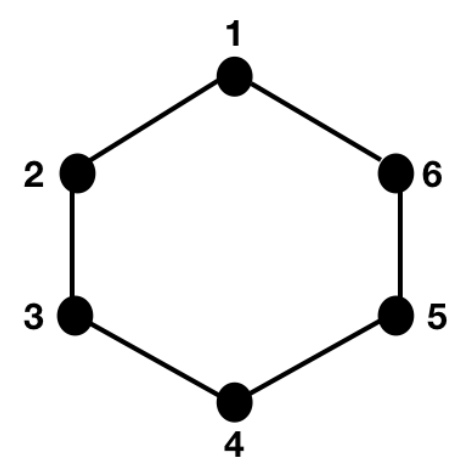

Figure 1.1: A labeled 6-cycle, $C_{6}$

is a suicide and the cops win in round 0 . If the robber chooses vertex 2 or 6 then the robber might be captured in the next round. So, by choosing 3,4 , or 5 the robber can evade the capture in round 1. So, maintaining distance two from the cop in every step is sufficient for the robber to win. But if we put 
two cops in round 0 , one cop on vertex 1 can stay there and another cop can visit the vertices one by one. So at some point, cops and robber will occupy the same vertex and then cops win.

The cops and robber game was first introduced by Quilliot [15] and then independently considered by Nowakowski and Winkler [13]. In the classical cops and robber game in [13, 15], both opponents have full information about each other's location as well as the structure of the graph. In those first research works, they focus on cop win graphs; they characterize those graphs where one cop is sufficient to capture the robber. Based on those, Clarke and MacGillivary [5] characterized $k$-cop win graphs; that is, graphs where $k$ cops are required. They also showed that even $k=2$ is nontrivial. Many variants of cops and robber have been considered. Those variants evolved from imposing restrictions on cops' movement or limiting cops' knowledge of the robber's position. Zero-visibility cops and robber games is an example of limiting cops' knowledge [6]. The zero-visibility cops and robber game was introduced by Tošić [17], which can be considered as a hybrid of the cops and robber game [13, 15] and the edge searching model [12]. Like the cops and robber game, the cops and robber take turns alternatively and each individual moves from the current vertex to one of its neighbours. Like the edge searching model, the robber is invisible. Tošić characterized the zero-visibility cop-win graphs for paths, cycles, complete graphs, and complete bipartite graphs [17. Dereniowski et al. [8] established a relationship between the zero-visibility cop number and the pathwidth of a graph. They introduced a monotonic version of the zero-visibility cops and robber model and gave an upper bound and 
a lower bound on the monotonic zero-visibility cop number. Tang [16] gave a quadratic time algorithm for computing the zero-visibility cop number of trees. Dereniowski et al.(7) improved this result by presenting a linear-time algorithm. They also proved that the problem of determining the zero-visibility cop number of a graph is NP-complete. Recently, Xue et al. 18 proposed a partition method for proving lower bounds for the zero-visibility cops and robber game. They also investigated the monotonic zero-visibility cop number of graph products. Xue et al. gave lower bounds and upper bounds for the zero-visibility cop number of graph joins, lexicographic products of graphs, complete multipartite graphs and split graphs [19].

Clarke et al. 4] considered a variation of the cops and robber game, called the $\ell$-visibility cops and robber game. This game has the same setting as the cops and robber game except that the cops have the information about the location of the robber only when the distance between the cops and the robber is less than or equal to $\ell$. There are two sub-tasks for cops: seeing and capturing. In the first phase, the cops move within the distance $\ell$ of the robber and in the second phase, they capture the robber. They characterize those trees for which $k$ cops are sufficient to guarantee capture of the robber for all $\ell \geq 1$. When $\ell=1$, the game is called the one-visibility cops and robber game, which was considered by Yang in [23]. A brief survey of cops and robber model is given in [2].

Let us play a 1-visibility cops and robber game on the graph in Figure 1.2. If we place a cop $\lambda_{1}$ on vertex 1 then vertex 1 is occupied and as vertex 2,3 and 4 is seen by $\lambda_{1}$ so robber will not occupy those vertices at round 0. Suppose, 


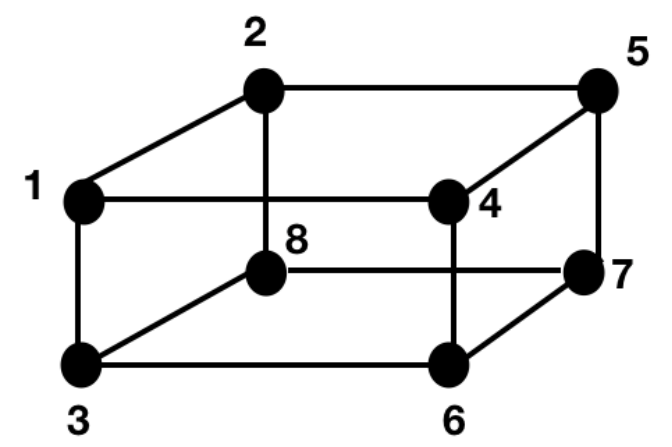

Figure 1.2: A labeled cube $Q_{3}$

the robber occupies vertex 6 . In round 1 , if the cop $\lambda_{1}$ moves to an adjacent vertex, say 4 then vertices 1,5 and 6 are seen by cop $\lambda_{1}$ and the robber is within distance 1 of the cop. But if the robber occupies vertex 7 at round 0 then it is safe as whatever vertex is chosen by $\lambda_{1}$ at round 1 vertex 7 will be at distance 2. To capture the robber we need at least two cops here. We keep one cop $\lambda_{1}$ vibrating between vertex 1 and 4 . So, vertices $1,2,3,4,5,6$ are either occupied or seen by cop $\lambda_{1}$. Only vertex 7 or 8 could be occupied by a robber. If we place another $\operatorname{cop} \lambda_{2}$ on vertex 8 and the robber occupies vertex 7 then it is at distance 1 of cop $\lambda_{2}$ and will be captured in round 1 . So here we need two cops to see and capture the robber.

\subsection{Main Contribution}

In this thesis, we investigate one-visibility cops and robber game on trees. Our first contribution is to develop a theorem to find a lower bound of one-visibility cop number of trees. We find the smallest number of cops that suffices to clear 
a tree. This theorem leads us to the key concepts needed for the clearing strategy of a tree, the concepts of "Hub", "Avenue", and "Street". We use the theorem to find the one-visibility cop number of a perfect binary tree and $k$-ary tree as a function of the height of the tree. This theorem is also used in the rest of the thesis to obtain other results. Our second contribution is to provide clearing strategies for trees containing hub, avenue, and street.

Our third contribution is that we propose a linear time algorithm to calculate the labels of vertices of a given tree. The complexity of our algorithm is $O(n)$. The first component in the label of the root represents the cop number of the tree. The label of each vertex is needed for developing an optimal clearing guide of a tree. We propose an optimal clearing guide to clear a given tree. That is our fourth contribution.

Our fifth contribution is to find the relations between zero-visibility cop number and one-visibility cop number of a tree $T$. We prove that the ratio of a zero-visibility cop number and one-visibility cop number of a tree $T$ is less than or equal two. We also present some interesting relations between zero-visibility cop number and one-visibility cop number of some special trees such as perfect binary tree and $k$-ary tree which is our sixth contribution.

\subsection{Organization}

The structure of the thesis is organized as follows. In Chapter 2, we give definitions and introduce common notations used in this thesis. We also discuss 
and give examples of one-visibility cops and robber game on trees. The results of this thesis are divided into three chapters. In Chapter 3, we explain an essential theorem to calculate the lower bound of cop numbers on trees. We also provide search strategies according to the structure of the tree in Chapter 3. An algorithm for calculating the labels of all vertices of an input tree is given in Chapter 4. The correctness and complexity of the algorithm are also provided in this chapter. We also provide an optimal clearing guide for a given tree. In Chapter 5, we examine relations between one- and zero-visibility cop numbers of a tree. We provide the ratio between zero-visibility cop number and one-visibility cop number. We also provide relations between one-visibility cop number and zero-visibility cop number of some special trees. Finally, in Chapter 6, we present a conclusion and future work. 


\section{Chapter 2}

\section{Preliminaries}

In section 2.1 we introduce some notations and definitions used in this thesis and in section 2.2 we discuss about one-visibility cops and robber game.

\subsection{Definitions}

We consider here a special case of limited visibility cops and robber game which is referred to as one-visibility cops and robber game. In this thesis, we explore this game on trees. Let $G$ be a graph. The vertex set of $G$ is denoted by $V(G)$. Let $u, v \in V(G)$. If $u$ and $v$ are joined by an edge, we say that $u$ and $v$ are adjacent or $u$ and $v$ are neighbours. We use $u v$ to denote an edge with endpoints $u$ and $v$. Similarly, we use $u_{1} \cdots u_{m}$ to denote a path with end vertices $u_{1}$ and $u_{m}$. The length of a path is the number of edges on the path. The distance between $u$ and $v$, denoted by $\operatorname{dist}_{G}(u, v)$, is the length of the 
shortest path between $u$ and $v$ in $G$. Let $H$ be a subgraph of $G$. The distance between $u$ and $H$ is defined to be $\operatorname{dist}_{G}(u, H):=\min \left\{\operatorname{dist}_{G}(u, v) \mid v \in V(H)\right\}$. Let $U \subseteq V(G)$. The distance between $u$ and $U$ can be defined similarly. The neighbourhood of $v$ is the set $N_{G}(v):=\left\{u \in V(G) \mid \operatorname{dist}_{G}(u, v)=1\right\}$. The closed neighbourhood of $v$ is the set $N_{G}[v]:=\left\{u \in V(G) \mid \operatorname{dist}_{G}(u, v) \leq 1\right\}$. For $k \geq 0$, we generalize this concept to the $k$-th closed neighbourhood of $v$, which is the set

$$
N_{G}^{k}[v]:=\left\{u \in V(G) \mid \operatorname{dist}_{G}(u, v) \leq k\right\}
$$

The closed neighbourhood of $U$ is defined as the set $N_{G}[U]=\{u \in V(G) \mid$ $\left.\operatorname{dist}_{G}(u, U) \leq 1\right\}$. If there is no ambiguity we omit the subscript $G$ and simply use $\operatorname{dist}(u, v), N^{k}[v], N[U]$.

The degree of $v$ is the number of edges incident on $v$, denoted $\operatorname{deg}_{G}(v)$. A leaf is a vertex that has degree one. For $U \subseteq V(G)$, we use $G[U]$ to denote the subgraph induced by $U$, which consists of all vertices of $U$ and all of the edges that connect vertices of $U$ in $G$. We use $G-U$ to denote the subgraph $G[V(G)-U]$. If $U$ contains a single vertex $u$, then for simplicity, we use $G-u$ for $G-\{u\}$. Let $E^{\prime}$ be a subset of edges of $G$. Similarly, we use $G-E^{\prime}$ to denote the subgraph obtained from $G$ by deleting all edges of $E^{\prime}$ from $G$.

A rooted tree is a tree where a single vertex is marked as the root. Let $T^{[r]}$ denote a rooted tree $T$ with root $r$. Every vertex $v \neq r$ of the tree is connected with root $r$ by a unique path where the parent of $v$ is the sole neighbour of $v$ in the unique path. If $u$ is the parent of $v$, then $v$ is a child of $u$. For a vertex 
$v \in V\left(T^{[r]}\right)$, if a vertex $u$ is on the unique path from $r$ to $v$, then we say that $v$ is a descendant of $u$, and $u$ is an ancestor of $v$. For a vertex $v \in V\left(T^{[r]}\right)$, we will use $T^{[v]}$ to denote the subtree of $T^{[r]}$ induced by $v$ and all its descendants, where $v$ is the root of this subtree. We will extensively use the notation of $T^{[v]}-v$ in Chapter 4 to denote the forest induced by $V\left(T^{[v]}\right)-\{v\}$, where $T^{[v]}$ is a rooted subtree of $T^{[r]}$. Note that each component in the forest $T^{[v]}-v$ is rooted at the vertex that is a child of $v$ in $T^{[v]}$. We will also use $T^{[r]}-v$ to denote the forest induced by $V\left(T^{[r]}\right)-\{v\}$.

\subsection{One-Visibility Cops and Robber}

The one-visibility cops and robber game is played on a graph by two players: cop player and robber player. The cop player controls a set of cops and the robber player controls a single robber. The robber has full information about the locations of all cops, but the cops have the information about the location of the robber only when there is a cop whose distance to the robber is at most one. The game is played over a sequence of rounds. Each round consists of a cops' turn followed by a robber's turn. At round 0, the cops are placed on a set of vertices and then the robber is placed on a vertex. At each of the following rounds, the cops move first and the robber move next. At round $i$, $i \geq 1$, each cop either moves from the current vertex to a neighbouring vertex or stays still, then the robber does the same. The cops see the robber if the closed neighbourhood of the cops contains the robber. The cops capture the robber if one of them occupies the same vertex as the robber. If this happens 
in a finite number of rounds, then the cops win; otherwise, the robber wins. The one-visibility cop number of a graph $G$, denoted by $c_{1}(G)$, is the minimum number of cops required to capture the robber on $G$.

If $G$ is not connected, from the above definition, we know that $\mathrm{c}_{1}(G)$ is the sum of the one-visibility cop number of each component of $G$. We define $c_{1}^{*}(G)$ to be the largest possible $c_{1}\left(G^{\prime}\right)$, where $G^{\prime}$ is a component in $G$.

The zero-visibility cops and robber game has the same setting as the onevisibility cops and robber game except that the cops have no information about the location of the robber at any time, i.e., the robber is invisible to the cops. However, if a cop occupies the same vertex as the robber at some moment, then the robber is captured. The zero-visibility cop number of a graph $G$, denoted by $\mathrm{c}_{0}(G)$, is the minimum number of cops required to capture the robber on $G$.

Throughout the thesis, we assume that both cops and the robber play optimally, which means the cops want to capture the robber as soon as possible while the robber wants to make the number of rounds of the game as large as possible. We sometimes use 1-visibility for "one-visibility" and 0-visibility for "zero-visibility".

Note that in a tree, once the robber has been seen by a cop, this cop can capture the robber in a finite number of rounds by moving to the vertex occupied by the robber when he was seen and eventually forcing the robber to a leaf. The next observation is from [4].

Proposition 2.1. Suppose that the 1-visibility cops and robber game is played 
on a tree. If the robber is seen by a cop at some moment, then the robber can be captured by this cop in a finite number of rounds.

From Proposition 2.1, we know that seeing implies capturing on trees.

A cops' strategy is a sequence of cops' actions to capture the robber. A clearing guide is a sequence of cops' actions and by following those actions the cop player can clear a tree. We say that a cop vibrates between two adjacent vertices $x$ and $y$ for a consecutive sequence of rounds if in these rounds, the cop alternates two actions: "sliding from $x$ to $y$ " and "sliding from $y$ to $x$ ". We define a cop as vibrating cop if the cop vibrates between two adjacent vertices $x$ and $y$ for more than two consecutive rounds.

We say a subgraph is cleared at some moment if it is certain that this subgraph does not contain the robber at the moment; otherwise, the subgraph is contaminated. Another important concept is recontamination of a vertex. If a vertex is defined clean by cops but later the robber can occupy this cleaned vertex then the vertex is recontaminated and this situation is called recontamination.

In Figure 2.1, let $T$ be a tree formed by the solid edges and the dotted edge, and let $T^{\prime}$ be a subtree of $T$ formed by only the solid edges. Lets play one visibility cops and robber game on tree $T$ given in Figure 2.1. First, place one $\operatorname{cop} \lambda_{1}$ on $a_{1}$. The cop does not need to visit the leaf as the visibility is one and the cop can see the robber if the robber is on the leaf. Then $\lambda_{1}$ moves towards $a$ and clear all the vertices on the path $a_{1} \ldots a$. Then $\lambda_{1}$ moves towards $a_{2}$ and when the cop is at $a_{2}$ then vertex $a$ is unobserved for two subsequent 


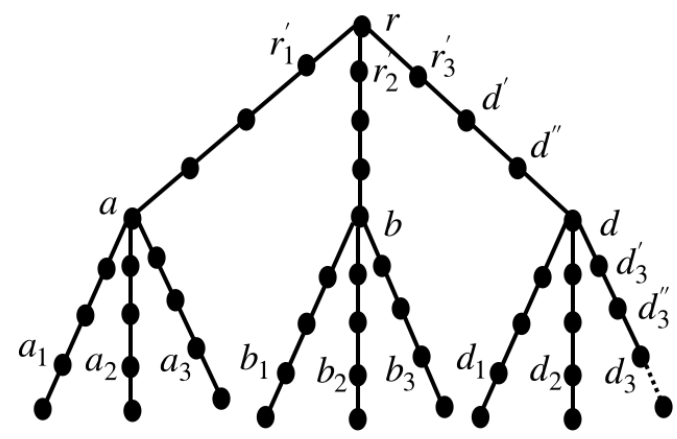

Figure 2.1: $c_{1}(T)=3$ and $c_{1}\left(T^{\prime}\right)=2$

cops terms and the robber can pass through $a$ to the cleared vertices. So, we need to guard the cleared vertices and for this, we need another cop $\lambda_{2}$ on $a$. So, $\lambda_{2}$ stays on $a$ and $\lambda_{1}$ clears the descendants of $a$. After clearing all the descendants of $a$ both $\lambda_{1}$ and $\lambda_{2}$ move towards $r$ along the path $a \ldots r$. Now, the cop $\lambda_{2}$ can vibrate between $d^{\prime}$ and $d^{\prime \prime}$ to protect $d$. Then vertex $r$ is unobserved for two subsequent cops' terms. So, the robber can recontaminate the cleared vertices by passing through $r$. So, we need another cop $\lambda_{3}$ to stay on $r$. The cop $\lambda_{2}$ can stay on $d$ or can vibrate between $d^{\prime}$ and $d^{\prime \prime}$ and $\lambda_{1}$ can clear the descendants of $d$. After clearing all the descendants of $d$ both $\lambda_{1}$ and $\lambda_{2}$ return to $r$ through $d \ldots r$. The rest of the dirty vertices can be cleared similarly. So, we need three cops to clear tree $T$.

Now we show that computing the one-visibility cop number of a given tree $T$ is a tricky algorithmic problem with an example. It is easy to see that $c_{1}(T)=3$, but it is probably not immediately obvious that $c_{1}\left(T^{\prime}\right)=2$. Now we show how we can clear the tree $T^{\prime}$ with two cops. As the subtree of $T^{\prime}$ rooted at $r_{1}^{\prime}$ is symmetrical to the subtree of $T$ rooted at $r_{1}^{\prime}$, two cops can clear the subtree 
of $T^{\prime}$ rooted at $r_{1}^{\prime}$. After that both $\lambda_{1}$ and $\lambda_{2}$ move to $r$. The cop $\lambda_{2}$ vibrates between $r_{3}^{\prime}$ and $d^{\prime}$ to guard the cleared vertices and $\lambda_{1}$ moves to $d$. At first $\lambda_{1}$ moves to $d_{1}$. Then $\lambda_{1}$ returns to $d$ through $d_{1} \ldots d$. After that, $\lambda_{1}$ moves to $d_{3}^{\prime}$ and then to $d_{3}^{\prime \prime}$ and clear all the rightmost descendants of $d$. In the meantime if the robber moves to $d$ then the cop can see the robber in the next round and can capture the robber in a finite number of rounds. After that, $\lambda_{1}$ clears the remaining dirty vertices and returns to $d$. As all the descendants of $r_{3}^{\prime}$ are now cleared both $\lambda_{1}$ and $\lambda_{2}$ return to $r$. Now both $\lambda_{1}$ and $\lambda_{2}$ move towards $b$ through $r \ldots b$. As all the descendants of both $r_{1}^{\prime}$ and $r_{3}^{\prime}$ are cleared, two cops can clear the descendants of $b$ by utilizing the same strategy applied for clearing the descendants of $a$. So two cops can clear $T^{\prime}$. 


\section{Chapter 3}

\section{Lower Bound on $\mathrm{c}_{1}(T)$}

In this Chapter, we show a structural theorem to provide a lower bound of cop number of a given tree $T$. We introduce different structures of a tree and provide strategies to clear trees according to their structures. We begin by proving some useful and essential lemmas for lower bound theorem.

\subsection{Lower Bound Theorem}

The notion of subtree plays an important role in constructing our structure theorem. Let $T=(V, E)$ be a tree. We here prove that for any subtree $H$ of $T, \mathrm{c}_{1}(H) \leq \mathrm{c}_{1}(T)$. The idea of the proof of this result is similar to Lemma 4.2 in $[8]$.

Lemma 3.1. For a tree $T$ and a subtree $H$ of $T, \mathrm{c}_{1}(H) \leq \mathrm{c}_{1}(T)$.

Proof. Suppose that $\mathrm{c}_{1}(T)=k$. We show that $\mathrm{c}_{1}(H) \leq k$. We consider a pair 
of 1-visibility games played in parallel on $T$ and $H$. Suppose the strategy to play the game on $T$ with $k$ cop is $S_{T}$. Now we produce a strategy $S_{H}$ to play the game on $H$. The $k$ cops play according to a winning strategy on $T$. The cops move in $S_{H}$ depends on the cops move in $S_{T}$. For each cop $\lambda_{j}, 1 \leq j \leq k$, if the cop $\lambda_{j}$ is initially placed in $H$, in $S_{H}$ we place the cop $\lambda_{j}$ in $H$. We do the following for each cop's move in $S_{T}$,

- In $S_{T}$, whenever a cop moves within $H$, in $S_{H}$ the corresponding cop also moves in $H$;

- In $S_{T}$, whenever a cop enters $H$, in $S_{H}$ we place the corresponding cop on $H$;

- In $S_{T}$, whenever a cop exits $H$, in $S_{H}$ the corresponding cop stays on $H$.

Since $S_{T}$ is a successful strategy on $T$, the strategy described above also successfully cleans $H$. Therefore, $\mathrm{c}_{1}(H) \leq \mathrm{c}_{1}(T)$.

Note that this Lemma does not hold for general graphs. For example, for any integer $k \geq 2$, we can apply Theorem 3.5 in this section to construct a tree $T$ with $\mathrm{c}_{1}(T) \geq k$, but for any complete graph $K_{n}$ (with $\left.n \geq|V(T)|\right)$, we have $c_{1}\left(K_{n}\right)=1$.

Lemma 3.2. Let $T$ be a tree and $v \in V(T)$. Let $H$ be a component in the forest $T-v$. If $\mathrm{c}_{1}(H)=k$, then in any cops' strategy to clear $T$, there is a moment at which at least $k$ cops are on $N_{T}[V(H)]$. 
Proof. Let $u$ be the unique neighbour of $v$ in $H$, and let $H_{v}$ be the subtree obtained by adding the vertex $v$ and edge $u v$ to $H$. Note that $N_{T}[V(H)]=$ $V\left(H_{v}\right)$. Assume, for the sake of contradiction, that there is a cops' strategy $S_{T}$ for clearing $T$ such that at any moment there are at most $k-1$ cops on $H_{v}$. We construct a pseudo strategy $S$ to clear $H_{v}$ as follows: In $S_{T}$, whenever a cop is initially placed in $H_{v}$, in $S$ we place the corresponding cop on the same vertex. In $S_{T}$, whenever a cop moves within $H_{v}$, in $S$ the corresponding cop has the same action in $H_{v}$. In $S_{T}$, whenever a cop enters $H_{v}$, in $S$ we place the corresponding cop on $v$. In $S_{T}$, whenever a cop leaves $H_{v}$, in $S$ we remove the corresponding cop from $v$. We can easily modify the pseudo strategy $S$ to a cops' strategy $S^{\prime}$ for $H_{v}$ such that the number of cops used in $S^{\prime}$ is the same as that in $S$. From the assumption, at any moment in $S$ there are at most $k-1$ cops on $H_{v}$. So $H_{v}$ can be cleared by $S^{\prime}$ using at most $k-1$ cops. It follows from Lemma 3.1 that $H$ is cleared by at most $k-1$ cops. Thus $\mathrm{c}_{1}(H) \leq k-1$, which is a contradiction.

Lemma 3.3. Let $T$ be a tree and $v \in V(T)$. Let $H$ be a component in the forest $T-v$. If $\mathrm{c}_{1}(H)=k$, then there is a strategy to clear $T$ such that at any moment there are at most $k$ cops on $N_{T}[V(H)]$.

Proof. Let $u$ be the unique neighbour of $v$ in $H$, and let $H_{v}$ be the subtree obtained by adding the vertex $v$ and edge $u v$ to $H$. Note that $N_{T}[V(H)]=$ $V\left(H_{v}\right)$. Assume that in any cops' strategy $S_{T}$ for clearing $T$ there is a moment such that at least $k+1$ cops are on $H_{v}$. But from Lemma 3.2 we know if $\mathrm{c}_{1}(H)=k$, then in any cops' strategy to clear $T$, there is a moment at which at least $k$ cops are on $H_{v}$. So we can say in this case $\mathrm{c}_{1}(H)=k+1$, which is 
a contradiction.

Note that the condition in Lemma 3.3 does not hold for general subtree $H$ of $T$. For example consider the tree $T$ in Figure 3.1, where $H$ is a subtree of $T$ consisting of vertices in the dotted region. We can see in any cops strategy to clear $T$ there is a moment at which 2 cops are on $N_{T}[V(H)]$.

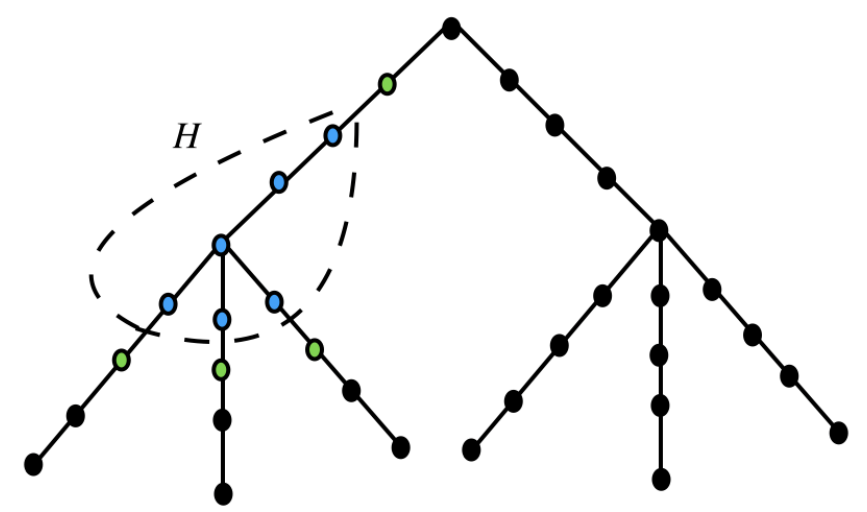

Figure 3.1: A tree $T$ with $c_{1}(T)=2$ and $c_{1}(H)=1$.

Now consider the Figure 3.2, where the subtree $H$ of $T$ is indicated by the dotted region and $c_{1}(T)=3$ and $c_{1}(H)=1$. In that figure in any cops strategy to clear $T$ there is a moment at which 2 cops are on $V(H)$.

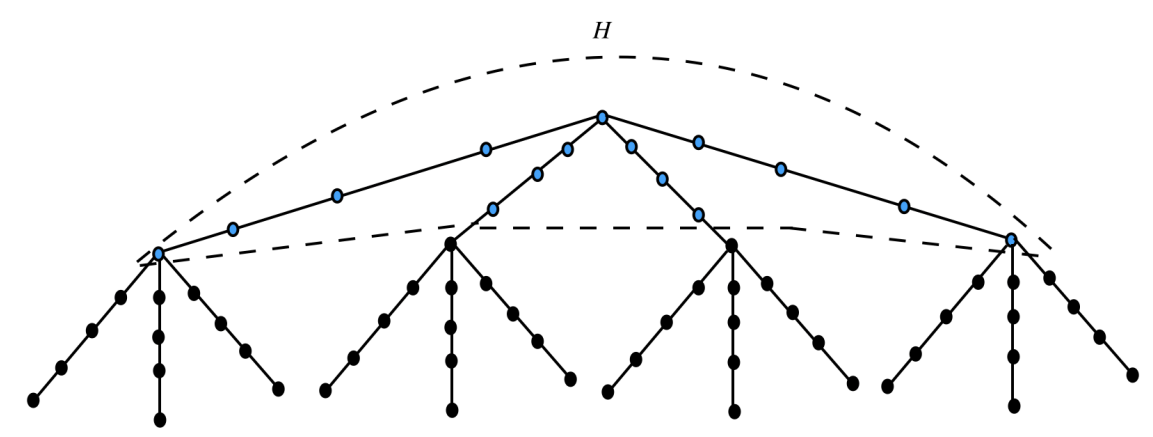

Figure 3.2: A tree $T$ with $c_{1}(T)=3$ and $c_{1}(H)=1$. 
Let $T$ be a tree and let $S$ be a cops' strategy that clears $T$ in $m$ rounds. Recall that a vertex $v$ is contaminated at some moment if $v$ is occupied by the robber or the cops do not know if the robber is on $v$ at the moment. Let $V_{b}^{i}$, $1 \leq i \leq m$, be the set of contaminated vertices of $T$ before the cops' move in round $i$, let $V_{a}^{i}, 1 \leq i \leq m$, be the set of contaminated vertices of $T$ just after the cops' move in round $i$, and let $V_{c}^{i}, 0 \leq i \leq m$, be the set of vertices of $T$ occupied by cops after the cops' move in round $i$. In the next lemma we show that the robber's territory is not far away from the set of vertices occupied by cops.

Lemma 3.4. Let $T$ be a tree and let $S$ be a cops' strategy that clears $T$ in $m$ rounds. Then

(i) for every component $H$ of $T\left[V_{b}^{i}\right], 1 \leq i \leq m$, there is a vertex $v \in V_{c}^{i-1}$ such that $\operatorname{dist}_{T}(v, H) \leq 2$; and

(ii) for every component $H$ of $T\left[V_{a}^{i}\right], 1 \leq i \leq m-1$, there is a vertex $v \in V_{c}^{i}$ such that $\operatorname{dist}_{T}(v, H) \leq 3$.

Proof. Let $V_{r}^{i}, 0 \leq i \leq m-1$, be the set containing a single vertex that is occupied by the robber after the robber's move in round $i$. At round 0 , cops are placed on vertices of $V_{c}^{0}$, and then the robber is placed on the vertex in $V_{r}^{0}$. So at round $1, V_{b}^{1}=\left(V(T)-N_{T}\left[V_{c}^{0}\right]\right) \cup V_{r}^{0}$, and thus, statement (i) holds. Since every cop can move to a neighbouring vertex or stays still, it is easy to see that statement (ii) also holds. Suppose both statements hold at round $i$. We will show that both of them are true at round $i+1$. 
At round $i+1$, we have $V_{b}^{i+1}=\left(N_{T}\left[V_{a}^{i}\right]-N_{T}\left[V_{c}^{i}\right]\right) \cup V_{r}^{i}$. Let $H$ be a component of $T\left[V_{b}^{i+1}\right]$. Since $V_{a}^{i} \subseteq V_{b}^{i+1}$, there exists a component $H^{\prime}$ in $T\left[V_{a}^{i}\right]$ such that $H^{\prime}$ is a subgraph of $H$. By the assumption, there is $v \in V_{c}^{i}$ such that $\operatorname{dist}_{T}\left(v, H^{\prime}\right) \leq 3$. If $\operatorname{dist}_{T}\left(v, H^{\prime}\right) \leq 2$, then $\operatorname{dist}_{T}(v, H) \leq 2$. If $\operatorname{dist}_{T}\left(v, H^{\prime}\right)=3$, then there is a vertex $x \in N_{T}\left[V_{a}^{i}\right] \operatorname{such}$ that $\operatorname{dist}_{T}(x, v)=2$, and thus $x \in V_{b}^{i+1}$. Hence $\operatorname{dist}_{T}(v, H) \leq 2$ and so statement (i) is true. Just after the cops' move at round $i+1$, we have $V_{a}^{i+1}=\left(V_{b}^{i+1}-N_{T}\left[V_{c}^{i+1}\right]\right) \cup V_{r}^{i}$. Let $H$ be a component in $T\left[V_{a}^{i+1}\right]$. Since $V_{a}^{i+1} \subseteq V_{b}^{i+1}$, there is a component $H^{\prime \prime}$ in $T\left[V_{b}^{i+1}\right]$ such that $H$ is a subgraph of $H^{\prime \prime}$. By the above, there is $v \in V_{c}^{i} \operatorname{such}_{\text {that }} \operatorname{dist}_{T}\left(v, H^{\prime \prime}\right) \leq 2$. Then for any vertex $x \in N(v) \cap V_{c}^{i+1}$, $\operatorname{dist}_{T}(x, v) \leq 3$. Thus statement (ii) is true.

From the above two lemmas, we can show an essential theorem of this section.

Theorem 3.5. Let $T$ be a tree and let $k$ be a positive integer. If there is a vertex $v \in V(T)$ such that the forest $T-N_{T}^{3}[v]$ contains three components with cop number at least $k$ and the path in $T$ connecting any pair of these three components contains $v$, then $\mathrm{c}_{1}(T) \geq k+1$.

Proof. Let $X_{1}, X_{2}, X_{3}$ be three components in $T-N_{T}^{3}[v]$ with $c_{1}\left(X_{i}\right) \geq k$, $1 \leq i \leq 3$. For $1 \leq i \leq 3$, let $p_{i} \in V\left(X_{i}\right)$ with $\operatorname{dist}_{T}\left(p_{i}, v\right)=4$. Let $T_{1}$ be a subtree of $T$ which is formed from the disjoint union of $X_{i}, 1 \leq i \leq 3$, together with the paths of length four from $p_{i}$ to $v$ (see Figure 3.3). For the sake of contradiction, assume $T_{1}$ can be cleared by $k$ cops. Then there is a round $t_{1}$ such that just after the cops' move in this round, only one of the three 
components, say $X_{1}$, is cleared and it remains cleared through the following rounds. There is also a round $t_{2}$ such that just after the cops' move, one of the other two components, say $X_{2}$, is cleared. Since $\mathrm{c}_{1}\left(X_{2}\right) \geq k$, from Lemma 3.2 , there is a round $t_{3}$ between $t_{1}$ and $t_{2}$ such that at the moment $t^{*}$ just after the cops' move in round $t_{3}$, all $k$ cops are simultaneously present in $N_{T}\left[V\left(X_{2}\right)\right]$. As $X_{3}$ is contaminated at $t^{*}$ and $\operatorname{dist}_{T_{1}}\left(v, N_{T}\left[V\left(X_{2}\right)\right]\right)=3$, by Lemma 3.4 , $v$ is contaminated at $t^{*}$. So the robber will recontaminate $X_{1}$, which derives a contradiction. Therefore $c_{1}\left(T_{1}\right) \geq k+1$. It follows from Lemma 3.1 that $\mathrm{c}_{1}(T) \geq k+1$.

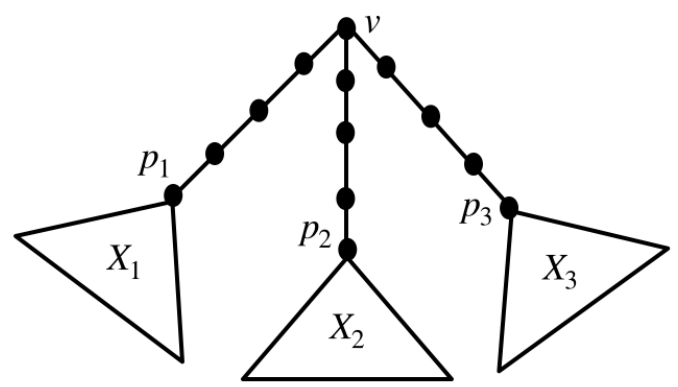

Figure 3.3: $T_{1}$ consists of $X_{1}, X_{2}, X_{3}$ and the paths to $v$.

\subsection{Cops' Strategies for Trees}

Definition 3.6. (hub, street, and avenue vertices) Suppose that $c_{1}(T)=$ $k$. A vertex $v$ is called a $h u b$ of $T$ if each component $X$ in the forest $T-N_{T}^{3}[v]$ has $\mathrm{c}_{1}(X)<k$. A vertex $v$ is called an avenue vertex of $T$ if $T-N_{T}^{3}[v]$ has two components with cop number $k$ satisfying that the path in $T$ connecting 


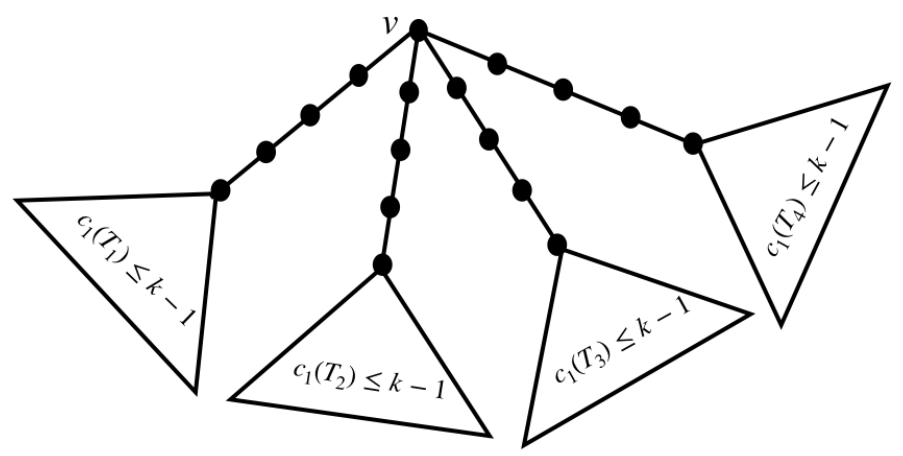

Figure 3.4: A tree $T$ with $\mathrm{c}_{1}(T)=k$, where $v$ is a hub.

them contains $v$ (see Figure 3.5). A vertex $v$ is called a street vertex of $T$ if $T$ does not have a hub or an avenue vertex, and $T-N_{T}^{2}[v]$ has two components with cop number $k$ such that the path in $T$ connecting them contains $v$ (see Figure 3.6.).

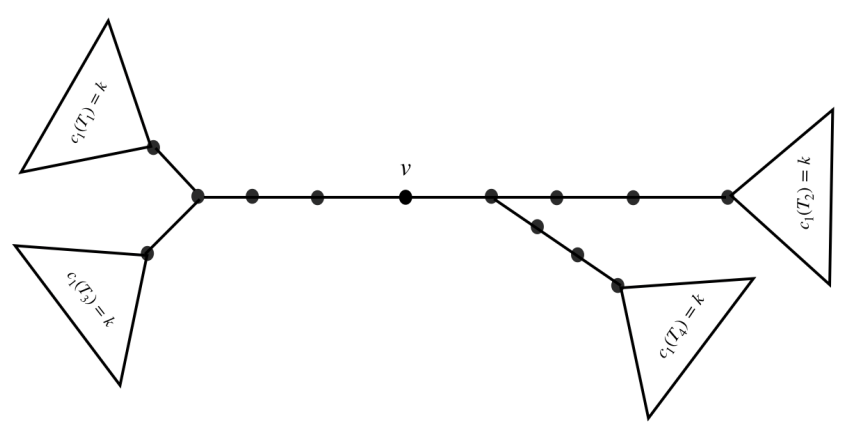

Figure 3.5: A tree $T$ with $c_{1}(T)=k$, where $v$ is an avenue vertex.

Theorem 3.7. Every tree contains a hub, an avenue vertex, or a street vertex.

Proof. Let $T$ be a tree with $\mathrm{c}_{1}(T)=k \geq 1$. If there is a vertex $v$ in $T$ such that $T-N_{T}^{3}[v]$ is empty, then $c_{1}(T)=1$ and $v$ is a hub of $T$. Suppose that there is no vertex $v$ in $T$ such that $T-N_{T}^{3}[v]$ is empty. 


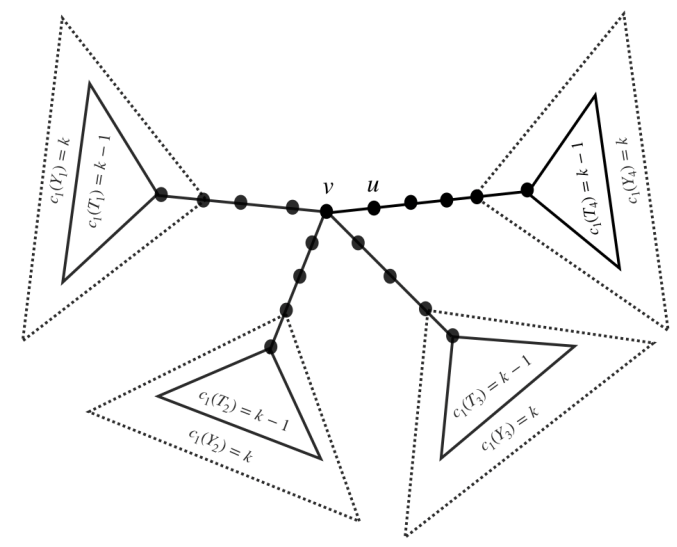

Figure 3.6: A tree $T$ with $\mathrm{c}_{1}(T)=k$, where $u$ and $v$ are street vertices.

If there is a vertex $v$ such that each component in $T-N_{T}^{3}[v]$ has cop number less than $k$, then by Definition $3.6 v$ is a hub.

If $T$ does not have a hub, then for every vertex $v$ in $T, T-N_{T}^{3}[v]$ must contain at least one component of cop number $k$. If there is a vertex $v$ such that $T-N_{T}^{3}[v]$ has three components with cop number $k$ and the path in $T$ connecting any pair of these three components contains $v$, then, by Theorem 3.5 , $\mathrm{c}_{1}(T) \geq k+1$. This is a contradiction as $\mathrm{c}_{1}(T)=k$.

If there is a vertex $u$ such that $T-N_{T}^{3}[u]$ has two components of cop number $k$ and the path between them contains $u$, then from Definition 3.6, $u$ is an avenue vertex.

Suppose that $T$ does not have a hub or an avenue vertex. Let $v$ be an arbitrary vertex in $T$. Then $T-N_{T}^{3}[v]$ has at least one component of cop number $k$, but it does not have two components with cop number $k$ such that the path between them contains $v$. So all components with cop number $k$ in 
$T-N_{T}^{3}[v]$ must be subgraphs of the same component in the forest $T-v$. Let $v^{\prime}$ be a neighbour of $v$ such that all components with cop number $k$ in $T-N_{T}^{3}[v]$ be subgraphs of the component in $T-v$ which contains $v^{\prime}$. This component is denoted by $T_{v v^{\prime}}$ which is associated with the edge $v v^{\prime}$. Since the number of vertices in $T$ is one more than that of edges, by the pigeonhole principle there must exist at least one edge $x y$ that is associated with two components $T_{x y}$ and $T_{y x}$. Thus $T-N_{T}^{3}[x]$ contains a set $F_{1}$ of components with cop number $k$ such that the path in $T$ between any pair of components from $F_{1}$ does not contain $x$; similarly, $T-N_{T}^{3}[y]$ contains a set $F_{2}$ of components with cop number $k$ such that the path in $T$ between any pair of components from $F_{2}$ does not contain $y$. Note that the components in $F_{1}$ and $F_{2}$ are subgraphs of components in $T-N_{T}^{2}[x]$. Hence $T-N_{T}^{2}[x]$ has two components with cop number $k$, where one is a supergraph of a component in $F_{1}$ and the other is a supergraph of a component in $F_{2}$, such that the path in $T$ connecting them contains $x$. From Definition 3.6, $x$ is a street vertex. Similarly, $y$ is also a street vertex.

From Definition 3.6 and the proof of Theorem 3.7, every tree has the following property.

Corollary 3.8. Every tree contains exactly one of the three kinds of vertices: (i) hubs, (ii) avenue vertices, and (iii) street vertices.

Proof. Let $T$ be a tree with $\mathrm{c}_{1}(T)=k$. Suppose we have an avenue vertex $v$ in $T$. As $T$ contains an avenue vertex by definition $T$ can not have a street vertex. Since a vertex $u$ of the form hub can not be on the avenue, and, if it were off the avenue, the component in $T-N^{3}[u]$ that included the avenue 
would have cop number $k$, contradicting the requirement for a hub that all component in $T-N^{3}[u]$ have cop number less than $k$.

Assume that $T$ contains two street vertices $u v$. By definition, $T$ can not have a hub or an avenue vertex.

Suppose that $T$ contains a hub $v$. If there exists an avenue vertex $u$ in $T$, at least one component in $T-N^{3}[v]$ must include that avenue, which contradicts with the requirement for a hub. The same applies to a street vertex. So if $T$ contains a hub then $T$ does not contain a street or an avenue vertex.

Note that a tree may have some vertices which are none of the three kinds in Corollary 3.8 (see Figure 3.7).

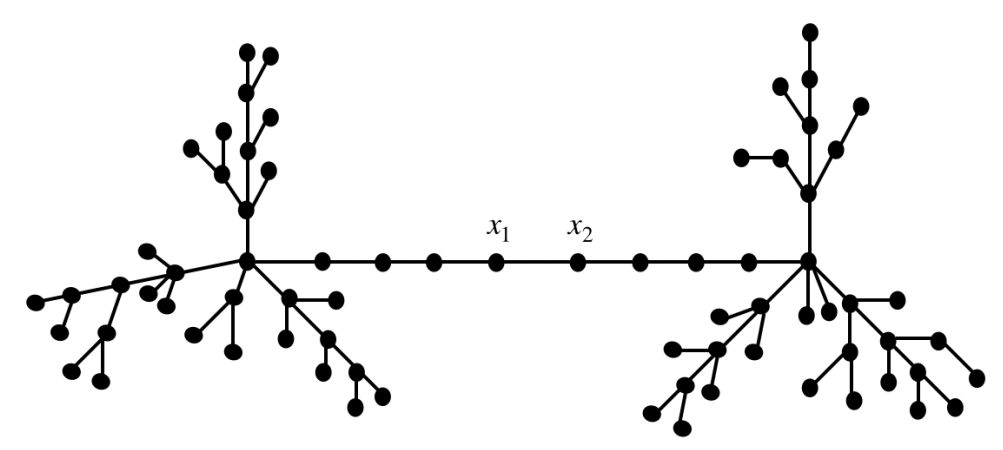

Figure 3.7: A tree $T$ with $\mathrm{c}_{1}(T)=2$, where $x_{1}$ and $x_{2}$ are avenue vertices of $T$ and all others are not hubs, street vertices or avenue vertices of $T$.

A graph could have many hubs and the distance between hubs could be arbitrarily large. Figure 3.8 shows an example where every vertex in the tree is a hub.

Lemma 3.9. If a tree contains hubs, then the subgraph induced by all hubs of the tree is connected. 


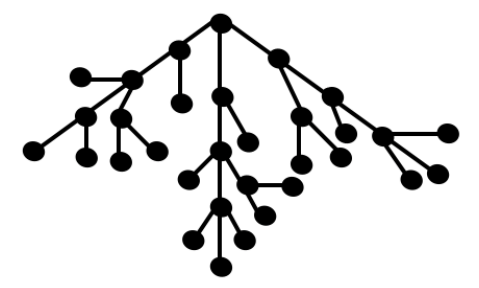

Figure 3.8: A tree $T$ with $c_{1}(T)=2$, in which every vertex is a hub.

Proof. Let $T$ be tree with $c_{1}(T)=k$ and let $V_{h}$ be the set of all hubs of $T$. Suppose $T\left[V_{h}\right]$ is not connected and $v_{1}$ and $v_{2}$ are two vertices of $V_{h}$ in two different components of $T\left[V_{h}\right]$. Then there is a vertex $u \notin V_{h}$ on the path in $T$ connecting $v_{1}$ and $v_{2}$. Since each component in $T-N_{T}^{3}\left[v_{i}\right], 1 \leq i \leq 2$, has cop number less than $k$, we know that $T-N_{T}^{3}[u]$ cannot contain a component with cop number $k$. So $u$ is a hub of $T$. This is a contradiction. Thus $T\left[V_{h}\right]$ is connected.

We now describe a cops' strategy to clear a tree that contains a hub.

function Hub-STRATEGy $(T, v)$

Let $T$ be a tree rooted at $v$ such that $c_{1}(T)=k$ and $v$ is a hub of $T$. Let $v_{1}, \ldots, v_{d}$ be all children of $v$. For each $v_{i}, 1 \leq i \leq d$, let $v_{i j}, 1 \leq j \leq m_{i}$, be all children of $v_{i}$.

1: Place all $k$ cops on $v$.

2: For each $v_{i}, 1 \leq i \leq d$, for each $v_{i j}, 1 \leq j \leq m_{i}$, let one cop vibrate between $v_{i}$ and $v_{i j}$, in the meantime, slide other cops into each subtree rooted at a grandchild of $v_{i j}$ until the subtree rooted at $v_{i j}$ is cleared.

3: Slide $k$ cops back to $v$.

Lemma 3.10. Let $T$ be a tree which does not have a hub or an avenue vertex. Then $T$ has exactly two street vertices, and they are adjacent to each other.

Proof. Let $\mathrm{c}_{1}(T)=k$. Since $T$ does not have a hub or an avenue vertex of $T$, 
from the proof of Theorem 3.7, there is at least one edge whose endpoints are street vertices of $T$. Following that proof, suppose there exists two edge $x_{1} x_{2}$ and $x_{3} x_{4}$ such that each $x_{i}, 1 \leq i \leq 4$, is a street vertex of $T$ and the path between $x_{1}$ and $x_{4}$ in $T$ contains $x_{2}$ and $x_{3}$. From Definition 3.6, $T-N_{T}^{2}\left[x_{1}\right]$ has two components with cop number $k$ such that the path in $T$ connecting them contains $x_{1}$; similarly, $T-N_{T}^{2}\left[x_{4}\right]$ has two components with cop number $k$ such that the path in $T$ connecting them contains $x_{4}$. Thus $T-N_{T}^{3}\left[x_{2}\right]$ has two components with cop number $k$ such that the path in $T$ connecting them contains $x_{2}$. Hence $x_{2}$ is an avenue vertex of $T$, which is a contradiction. So there is only one edge whose endpoints are street vertices of $T$.

The edge whose endpoints are street vertices of $T$ is called a street of $T$.

Lemma 3.11. Suppose that $T$ is a tree with $\mathrm{c}_{1}(T)=k$ and uv is a street of T. Let $T_{u v}$ be a component in the forest $T-u$ which contains a component of $T-N_{T}^{3}[u]$ with cop number $k$. Then $T_{u v}$ contains all components of $T-N_{T}^{3}[u]$ with cop number $k$, $u$ is a hub of $T-V\left(T_{u v}\right)$, and $v$ is a hub of $T_{u v}$.

Proof. Since $v$ is a street vertex of $T$, from Definition $3.6, T-N_{T}^{2}[v]$ has two components with cop number $k$ such that the path in $T$ connecting them contains $v$. Thus $T-N_{T}^{3}[u]$ has two components with cop number $k$ such that the path in $T$ connecting them contains $v$. Since $u$ is not an avenue vertex of $T$, all components with cop number $k$ in $T-N_{T}^{3}[u]$ must be contained in one component in the forest $T-u$, which must be $T_{u v}$. Therefore, the forest $T-V\left(T_{u v}\right)-N_{T}^{3}[u]$ does not have any component with cop number $k$.

Since $u$ is a street vertex of $T, T-N_{T}^{2}[u]$ has at least two components 
with cop number $k$ such that the path in $T$ connecting them contains $u$. So $T-V\left(T_{u v}\right)-N_{T}^{2}[u]$ has at least one component with cop number $k$, and thus $\mathrm{c}_{1}\left(T-V\left(T_{u v}\right)\right)=k$. Hence $u$ is a hub of $T-V\left(T_{u v}\right)$. Similarly, we can define $T_{v u}$, and symmetrically, $v$ is a hub of $T-V\left(T_{v u}\right)$. Note that $T-V\left(T_{v u}\right)=T_{u v}$. Therefore, $v$ is a hub of $T_{u v}$.

From Lemma 3.11, we have the following cops' strategy to clear a tree that contains a street.

function STREET-STRATEGY $(T, u v)$

Let $T$ be a tree with $\mathrm{c}_{1}(T)=k$, and let $u v$ be the street of $T$. Let $T_{u v}$ be the component in $T-u$ which contains all components of $T-N_{T}^{3}[u]$ with cop number $k$.

1: Place all $k$ cops on $u$; then Hub-Strategy $\left(T-V\left(T_{u v}\right), u\right)$.

2: Slide all cops from $u$ to $v$; then $\operatorname{Hub}-\operatorname{Strategy}\left(T_{u v}, v\right)$.

In the next lemma, we show that the subgraph induced by avenue vertices is a path.

Lemma 3.12. Let $A$ be the set of all avenue vertices of a tree $T$. Then $T[A]$ is a path.

Proof. Let $k=\mathrm{c}_{1}(T)$. Suppose that $T[A]$ is not connected. Let $v_{1}$ and $v_{2}$ be two vertices of $A$ in two different components of $T[A]$. Let $u \notin A$ be a vertex on the path in $T$ connecting $v_{1}$ and $v_{2}$. From Definition 3.6. $T-N_{T}^{3}\left[v_{i}\right]$, $1 \leq i \leq 2$, has two components with cop number $k$ such that the path in $T$ connecting them contains $v_{i}$. So $T-N_{T}^{3}[u]$ has two components with cop number $k$ satisfying that the path in $T$ connecting them contains $u$. Thus, $u$ is an avenue vertex, which is a contradiction. Hence $T[A]$ is connected. 
Suppose there is a vertex $v \in A$ that has at least three neighbours in $A$. Then $T-N_{T}^{3}[v]$ has at least three components with cop number $k$ such that the path in $T$ connecting any two of them contains $v$. It follows from Theorem 3.5 that $\mathrm{c}_{1}(T) \geq k+1$. This is a contradiction. Thus $T[A]$ does have a vertex of degree more than two. Therefore $T[A]$ is a path.

From Lemma 3.12 , it is easy to see that, the path $T[A]$ induced by the avenue vertices is unique. The path $T[A]$ in Lemma 3.12 is called the avenue of $T$.

Corollary 3.13. Let $T$ be a tree with $\mathrm{c}_{1}(T)=k$ and let $x_{1} \cdots x_{m}$ be the avenue of $T$. Then

(i) $x_{1}$ has a unique neighbour $v \neq x_{2}$ such that $T-N_{T}^{2}[v]$ has two components with cop number $k$ such that the path in $T$ connecting them contains $v$.

(ii) $v$ is a hub of $T-V\left(T^{*}\right)$, where $T^{*}$ is a component in $T-v$ that contains the avenue.

Proof. (i) As $x_{1}$ is an avenue vertex, by Definition 3.6, $T-N_{T}^{3}\left[x_{1}\right]$ has two components $T_{1}$ and $T_{2}$ with cop number $k$ such that the path in $T$ connecting them contains $x_{1}$. Without loss of generality, suppose that $x_{2}$ is on the path connecting $T_{2}$ to $x_{1}$. Let $v$ be a neighbour of $x_{1}$ on the path connecting $T_{1}$ to $x_{1}$. So $T_{1}$ is also a component in $T-N_{T}^{2}[v]$. Note that $T-N_{T}^{2}[v]$ has a component that contains $T_{2}$ as a subtree. Let this conponent be denoted by $T_{2}^{\prime}$. So $T-N_{T}^{2}[v]$ has two components, i.e., $T_{1}$ and $T_{2}^{\prime}$, with cop number $k$ such that the path in $T$ connecting them contains $v$. If $x_{1}$ has another neighbour 
$v^{\prime} \notin\left\{v, x_{2}\right\}$ such that $T-N_{T}^{2}\left[v^{\prime}\right]$ has two components with cop number $k$ such that the path in $T$ connecting them contains $v^{\prime}$, then from Theorem 3.5 . $\mathrm{c}_{1}(T) \geq k+1$. This is a contradiction. Thus $v$ is unique to have the property.

(ii) Note that $v$ is not an avenue vertex. So for any two components with cop number $k$ in $T-N_{T}^{3}[v]$, the path in $T$ connecting them does not contain $v$. So every component with cop number $k$ in $T-N_{T}^{3}[v]$ is a subtree of $T^{*}$. Hence $T-V\left(T^{*}\right)-N_{T}^{3}[v]$ does not contain any component with cop number $k$. Since $T-V\left(T^{*}\right)-N_{T}^{2}[v]$ contains the component $T_{1}$ with cop number $k$, we have $c_{1}\left(T-V\left(T^{*}\right)\right)=k$. Therefore $v$ is a hub of $T-V\left(T^{*}\right)$.

The vertex $v$ in Corollary 3.13 is called a pre-avenue vertex of $T$. If a tree has an avenue, then it has exactly two pre-avenue vertices, each of which is adjacent to an end vertex of the avenue.

From Lemma 3.12 and Corollary 3.13, we have the following cops' strategy to clear a tree that contains an avenue.

function Avenue-Strategy $(T, A)$

Let $T$ be a tree with $\mathrm{c}_{1}(T)=k$, and let $x_{1} \cdots x_{m}$ be the avenue of $T$, where $A=\left\{x_{1}, \ldots, x_{m}\right\}$ is the set of all avenue vertices of $T$. Let $x_{0}\left(\right.$ resp. $\left.x_{m+1}\right)$ be the pre-avenue vertex adjacent to $x_{1}\left(\operatorname{resp} . x_{m}\right)$. Let $Y_{0}\left(\operatorname{resp} . Y_{m+1}\right)$ be the component in $T-A$ which contains $x_{0}$ (resp. $\left.x_{m+1}\right)$. Let $Y_{i}, 1 \leq i \leq m$, be the component containing $x_{i}$ in the forest $T-\left\{x_{i-1} x_{i}, x_{i} x_{i+1}\right\}$.

1: Place all $k$ cops on $x_{0}$.

2: for $i:=0$ to $m$ do

3: $\quad$ Hub-Strategy $\left(Y_{i}, x_{i}\right)$; then slide all cops from $x_{i}$ to $x_{i+1}$.

4: $\operatorname{Hub-Strategy}\left(Y_{m+1}, x_{m+1}\right)$. 


\section{Chapter 4}

\section{One-Visibility Cop Number of a}

\section{Tree}

The main work of this section is to propose an $O(n)$ time algorithm to compute the cop number of a tree. We also propose an optimal clearing guide of a tree. First of all, we introduce some notations and definitions.

\subsection{Notations and Definitions}

From Section 2.2, we know $c_{1}^{*}(G)=\max \left\{\mathrm{c}_{1}\left(G^{\prime}\right) \mid G^{\prime}\right.$ is a component in $\left.G\right\}$. It is easy to see that if $G$ is connected, $c_{1}^{*}(G)=\mathrm{c}_{1}(G)$.

For simplicity, we will use $T^{[v]}-N^{3}[v]$ for $T^{[v]}-N_{T^{[v]}}^{3}[v]$, which is the forest obtained from the rooted tree $T^{[v]}$ by deleting the vertices of $N_{T^{[v]}}^{3}[v]$. Similarly, if there is no ambiguity we will simply use $\operatorname{dist}(u, v), N[v], N^{2}[v]$ 
and $N^{3}[v]$ without subscripts.

\section{Definition 4.1. ( $k$-pre-branching, $k$-weakly-branching, $k$-branching)}

Let $T^{[v]}$ be a rooted tree with $\mathrm{c}_{1}\left(T^{[v]}\right)=k \geq 1$. We call $v$ a $k$-pre-branching vertex if $c_{1}^{*}\left(T^{[v]}-N^{2}[v]\right)=k$ and $c_{1}\left(T_{2 v}^{[u]}\right)=k$, where $T_{2 v}^{[u]}$ is a tree obtained from two copies of $T^{[v]}$ by connecting each root $v$ to a new root $u$.

We call $v$ a $k$-weakly-branching vertex if one of the three forests, $T^{[v]}-v$, or $T^{[v]}-N[v]$, or $T^{[v]}-N^{2}[v]$, has exactly two components whose root is a $k$-pre-branching vertex in the component.

We call $v$ a $k$-branching vertex if $c_{1}^{*}\left(T^{[v]}-N^{2}[v]\right)=k$, the forest $T^{[v]}-N^{2}[v]$ has exactly one component whose root is a $k$-weakly-branching vertex in the component, and the forest $T^{[v]}-N^{3}[v]$ has no component whose root is a $k$-weakly-branching vertex.

Let $u$ be a child of $v$ in $T^{[v]}$. If $u$ is a $k$-pre-branching vertex (resp. $k$ weakly-branching vertex, $k$-branching vertex) in $T^{[u]}$, then we say that $u$ is a $k$-pre-branching child (resp. $k$-weakly-branching child, $k$-branching child) of $v$. Similarly, we can define the $k$-pre-branching descendant, $k$-weakly-branching descendant, and $k$-branching descendant of $v$.

Definition 4.2. Let $T^{[v]}$ be a rooted tree with $c_{1}\left(T^{[v]}\right)=k \geq 1$. The $k$-prebranching indicator $I_{\mathrm{pb}}^{k}(v)$ and the $k$-weakly-branching indicator $I_{\mathrm{wb}}^{k}(v)$ are defined to be:

$$
I_{\mathrm{pb}}^{k}(v)= \begin{cases}1, & \text { if } v \text { is a } k \text {-pre-branching vertex in } T^{[v]} \\ 0, & \text { otherwise. }\end{cases}
$$




$$
I_{\mathrm{wb}}^{k}(v)= \begin{cases}1, & \text { if } v \text { is a } k \text {-weakly-branching vertex in } T^{[v]} \\ 0, & \text { otherwise. }\end{cases}
$$

Definition 4.3. Let $T^{[v]}$ be a rooted tree with $\mathrm{c}_{1}\left(T^{[v]}\right)=k \geq 1$. The $k$-initialcounter $J^{k}(v)$ and the $k$-weakly-counter $J_{\mathrm{w}}^{k}(v)$ are defined as follows:

$$
J^{k}(v)= \begin{cases}0, & I_{\mathrm{pb}}^{k}(v)=0 \text { and } c_{1}^{*}\left(T^{[v]}-v\right)=k-1 \\ 1, & I_{\mathrm{pb}}^{k}(v)=0, c_{1}^{*}\left(T^{[v]}-N[v]\right)=k-1 \text { and } c_{1}^{*}\left(T^{[v]}-v\right)=k \\ 2, & I_{\mathrm{pb}}^{k}(v)=0, c_{1}^{*}\left(T^{[v]}-N^{2}[v]\right)=k-1 \text { and } c_{1}^{*}\left(T^{[v]}-N[v]\right)=k \\ 0, & \text { otherwise. }\end{cases}
$$

$$
J_{\mathrm{w}}^{k}(v)= \begin{cases}0, & \text { if } I_{\mathrm{wb}}^{k}(v)=1 \text { and } v \text { has exactly two } k \text {-pre-branching children } \\ & \text { and no } k \text {-weakly-branching child; } \\ 1, & \text { if } I_{\mathrm{wb}}^{k}(v)=1 \text { and } v \text { has exactly one } k \text {-weakly-branching child } \\ & \text { and this child } u \text { has } J_{\mathrm{w}}^{k}(u)=0 ; \\ 2, & \text { if } I_{\mathrm{wb}}^{k}(v)=1 \text { and } v \text { has exactly one } k \text {-weakly-branching child } \\ & \text { and this child } u \text { has } J_{\mathrm{w}}^{k}(u)=1 ; \\ 0, & \text { otherwise. }\end{cases}
$$

Remark. We know that the k-initial-counter $J^{k}(v)=0$ both for $I_{\mathrm{pb}}^{k}(v)=$ 0 and $I_{\mathrm{pb}}^{k}(v)=1$. But in the context of this thesis, it will not create any ambiguity.

Definition 4.4. (utmost- $k$-pre-branching vertex) Suppose $T^{[v]}$ is a rooted 
tree where the root $v$ is a $k$-pre-branching vertex. If $v$ does not have any $k$ pre-branching child then $v$ is the utmost- $k$-pre-branching vertex in $T^{[v]}$. If $v$ has only one $k$-pre-branching child $v_{1}$ then there exists a $y^{\text {th }}$ neighbour $z_{1}$ of $v$ such that for $y \geq 1, \#_{\mathrm{pb}}^{k}\left(T^{[v]}-N^{y-1}[v]\right)=1$, and $\#_{\mathrm{pb}}^{k}\left(T^{[v]}-N^{y}[v]\right)=0$. If $y=1$ then $v_{1}$ is the utmost- $k$-pre-branching vertex in $T^{[v]}$; otherwise $z_{1}$ is the utmost- $k$-pre-branching vertex in $T^{[v]}$. Note that in this case $z_{1}$ is also a descendant of $v_{1}$. All vertices on the path $z_{1} \ldots v$ are $k$-pre-branching vertices. If $v$ is a $k$-weakly-branching vertex in $T^{[v]}$ then $v$ has two $k$-pre-branching children say $v_{1}$ and $v_{2}$. Then there exists an utmost- $k$-pre-branching vertex $z_{1}$ in $T^{\left[v_{1}\right]}$ and an utmost- $k$-pre-branching vertex $z_{2}$ in $T^{\left[v_{2}\right]}$ and $z_{1}, z_{2}$ are two utmost-k-pre-branching vertices in $T^{[v]}$. If $v$ is a $k$-branching vertex in $T^{[v]}$ then $v$ has a $k$-weakly-branching descendant say $w$ and the two utmost- $k$-prebranching vertices $z_{1}, z_{2}$ in $T^{[w]}$ are two utmost- $k$-pre-branching vertices in $T^{[v]}$.

Definition 4.5. (road) If there exists an avenue in $T^{[v]}$ then from Corollary 3.13 we know there exist two pre-avenue vertices in $T^{[v]}$. Any path connecting two pre-avenue vertices is the road in $T^{[v]}$, where avenue vertices are internal road vertices; or any path connecting the utmost- $k$-pre-branching vertex and the root is a road in $T^{[v]}$, where the utmost- $k$-pre-branching vertex is a pre-avenue vertex and another pre-avenue vertex is on the road. If there exists a street in $T^{[v]}$ then any path connecting two street vertices is the road in $T^{[v]}$, or any path connecting the utmost- $k$-pre-branching vertex and the root is a road in $T^{[v]}$, where the utmost- $k$-pre-branching vertex and the parent of the utmost- $k$-pre-branching vertex are two street vertices. If there is no avenue or 
street vertex then the root $v$ is a hub as well as the road in $T^{[v]}$; or any path connecting the utmost-k-pre-branching vertex and the root is a road in $T^{[v]}$ where the utmost- $k$-pre-branching vertex is a hub in $T^{[v]}$. We denote a road in $T^{[v]}$ by $P_{T^{[v]}}$.

Remark. If there exists an utmost-k-pre-branching vertex $z_{1}$ in $T^{[v]}$ then one of the three cases can happen i) $z_{1}$ is a hub in $T^{[v]}$, ii) $z_{1}$ is one street vertex and the parent of $z_{1}$ is another street vertex in $T^{[v]}$ iii) $z_{1}$ is a pre-avenue vertex and one vertex on the path $v \sim z_{1}$ is another pre-avenue vertex in $T^{[v]}$.

Definition 4.6. (label $L_{T^{[v]}}(v)$, value $\left|L_{T^{[v]}}(v)\right|$ ) Let $T^{[v]}$ be a rooted tree. The label of $v$ in $T^{[v]}$, denoted by $L_{T^{[v]}}(v)$, is a sequence

$$
\left(s_{1}, v_{1}, P_{1} ; s_{2}, v_{2}, P_{2} ; \ldots ; s_{m}, v_{m}, P_{m} ; I_{\mathrm{wb}}^{s_{m}}(v), J_{\mathrm{w}}^{s_{m}}(v) ; I_{\mathrm{pb}}^{s_{m}}(v), J^{s_{m}}(v)\right),
$$

where $s_{i}, v_{i}$ and $P_{i}$ are defined in the following procedure:

1. If $T^{[v]}$ contains only one vertex, then $s_{1}=1, v_{1}=\perp, P_{1}=v, I_{\mathrm{wb}}^{s_{i}}(v)=$ $J_{\mathrm{w}}^{s_{i}}(v)=I_{\mathrm{pb}}^{s_{i}}(v)=J^{s_{i}}(v)=0$, and $\operatorname{return} L_{T^{[v]}}(v)=(1, \perp, v ; 0,0 ; 0,0) ;$ otherwise, set $i \leftarrow 1$ and $T_{1}^{[v]} \leftarrow T^{[v]}$.

2. Set $s_{i} \leftarrow \mathrm{c}_{1}\left(T_{1}^{[v]}\right)$. Then we have one of the following cases:

(a) If $v$ is an $s_{i}$-branching vertex in $T_{1}^{[v]}$, then $I_{\mathrm{wb}}^{s_{i}}(v)=J_{\mathrm{w}}^{s_{i}}(v)=$ $I_{\mathrm{pb}}^{s_{i}}(v)=J^{s_{i}}(v)=0$, and $P_{i}$ contains two utmost- $k$-pre-branching vertices $z_{1}$ and $z_{2}$ in $T_{1}^{[v]}$; and return $L_{T^{[v]}}(v)=\left(s_{1}, v_{1}, P_{1} ; \ldots ; s_{i}, v, z_{1} \sim z_{2} ; 0,0 ; 0,0\right)$. 
(b) If $v$ has an $s_{i}$-branching descendant in $T_{1}^{[v]}$, let $v_{i}$ be this vertex. Set $T_{1}^{[v]} \leftarrow T_{1}^{[v]}-V\left(T_{1}^{\left[v_{i}\right]}\right), i \leftarrow i+1$, and go back to Step 2 .

(c) If $v$ is an $s_{i}$-weakly-branching vertex in $T_{1}^{[v]}$, then $I_{\mathrm{pb}}^{s_{i}}(v)=J^{s_{i}}(v)=$ $0, I_{\mathrm{wb}}^{s_{i}}(v)=1$, and $J_{\mathrm{w}}^{s_{i}}(v)$ can be determined by Definition 4.3, and $P_{i}$ contains two utmost- $k$-pre-branching vertices $z_{1}$ and $z_{2}$ in $T_{1}^{[v]}$; $\operatorname{return} L_{T^{[v]}}(v)=\left(s_{1}, v_{1}, P_{1} ; \ldots ; s_{i}, \perp, z_{1} \sim z_{2} ; 1, J_{\mathrm{w}}^{s_{i}}(v) ; 0,0\right)$.

(d) If $v$ is an $s_{i}$-pre-branching vertex in $T_{1}^{[v]}$, then $I_{\mathrm{wb}}^{s_{i}}(v)=J_{\mathrm{w}}^{s_{i}}(v)=$ $J^{s_{i}}(v)=0, I_{\mathrm{pb}}^{s_{i}}(v)=1$, and either $v$ is the utmost- $k$-pre-branching vertex then return $L_{T^{[v]}}(v)=\left(s_{1}, v_{1}, P_{1} ; \ldots ; s_{i}, \perp, v ; 0,0 ; 1,0\right)$; or $P_{i}$ contains an utmost- $k$-pre-branching descendant $z_{1}$; then return $L_{T^{[v]}}(v)=\left(s_{1}, v_{1}, P_{1} ; \ldots ; s_{i}, \perp, v \sim z_{1} ; 0,0 ; 1,0\right)$.

(e) $I_{\mathrm{wb}}^{s_{i}}(v)=J_{\mathrm{w}}^{s_{i}}(v)=I_{\mathrm{pb}}^{s_{i}}(v)=0$, and $J^{s_{i}}(v)$ can be determined by Definition 4.3 , and $P_{i}$ contains the root $v$; return $L_{T^{[v]}}(v)=\left(s_{1}, v_{1}, P_{1} ; \ldots ; s_{i}, \perp, v ; 0,0 ; 0, J^{s_{i}}(v)\right)$.

The value of $L_{T^{[v]}}(v)$, denoted by $\left|L_{T^{[v]}}(v)\right|$, is equal to $s_{1}$. The cop number of $T^{[v]}$ is the first component $s_{1}$. The road in $T^{[v]}$ denoted by $P_{T^{[u]}}$ is equal to $P_{1}$.

If there is no ambiguity, we simply use $L(v)$ for $L_{T^{[v]}}(v)$. In Algorithm 1 , we will compute the cop number of subtrees in the reverse order $s_{m}, s_{m-1}, \ldots, s_{1}$. For convenience, in the rest of the thesis we let $t_{i}=s_{m-i+1}, x_{i}=v_{m-i+1}$, and $P_{i}=P_{m-i+1}$; i.e.,

$$
L(v)=\left(t_{m}^{v}, x_{m}^{v}, P_{m}^{v} ; \ldots ; t_{1}^{v}, x_{1}^{v}, P_{1}^{v} ; I_{\mathrm{wb}}^{t_{1}^{v}}(v), J_{\mathrm{w}}^{t_{1}^{v}}(v) ; I_{\mathrm{pb}}^{t_{1}^{v}}(v), J^{t_{1}^{v}}(v)\right)
$$


where the superscript $v$ in $t_{i}^{v}, x_{i}^{v}$ and $P_{i}^{v}$ are used to refer to the vertex $v$. Here $P_{i}^{v}$ is the road in tree $T^{[v]}-\bigcup_{j=i+1}^{m} V\left(T^{\left[x_{j}^{v}\right]}\right)$. So, $|L(v)|=t_{m}^{v}$. Note that only $x_{1}^{v}$ can be a " $\perp$ " sign, which means that neither $v$ is an $t_{1}^{v}$-branching vertex in $T^{[v]}-\bigcup_{i=2}^{m} V\left(T^{\left[x_{i}^{v}\right]}\right)$ nor it has an $t_{1}^{v}$-branching descendant in $T^{[v]}-\bigcup_{i=2}^{m} V\left(T^{\left[x_{i}^{v}\right]}\right)$. We call the first three components $\left(t_{m}^{v}, x_{m}^{v}, P_{m}^{v}\right)$ an item associated with $T^{[v]}$, and call each triple $\left(t_{i}^{v}, x_{i}^{v}, P_{i}^{v}\right), 1 \leq i<m$, an item associated with subtree $T^{[v]}-\bigcup_{j=i+1}^{m} V\left(T^{\left[x_{j}^{v}\right]}\right)$, where $t_{i}^{v}$ is called the key of the item and $x_{i}^{v}$ and $P_{i}^{v}$ are attributes.

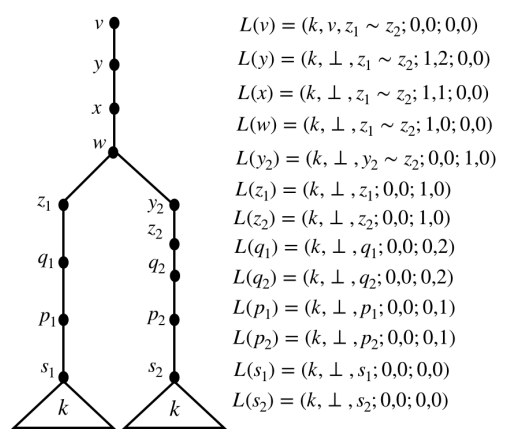

(a)

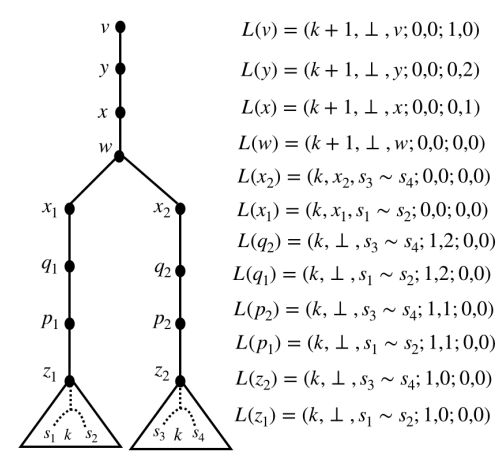

(b)

Figure 4.1: Labels of vertices

We explain labels with the following example.

Example 4.7. Consider the tree in Figure 4.1(a). Suppose that $v$ is the root and $L\left(s_{1}\right)=\left(k, \perp, s_{1} ; 0,0 ; 0,0\right)$ and $L\left(s_{2}\right)=\left(k, \perp, s_{2} ; 0,0 ; 0,0\right)$. Then $J^{k}\left(p_{1}\right)=J^{k}\left(p_{2}\right)=1$ and $J^{k}\left(q_{1}\right)=J^{k}\left(q_{2}\right)=2$. So $I_{\mathrm{pb}}^{k}\left(z_{1}\right)=I_{\mathrm{pb}}^{k}\left(z_{2}\right)=1$ and both $z_{1}$ and $z_{2}$ are $k$-pre-branching vertices with label $\left(k, \perp, z_{1} ; 0,0 ; 1,0\right)$ and $\left(k, \perp, z_{2} ; 0,0 ; 1,0\right)$ respectively. Note that $y_{2}$ is also a $k$-pre-branching vertex with label $\left(k, \perp, y_{2} \sim z_{2} ; 0,0 ; 1,0\right)$. The vertex $w$ is a $k$-weakly-branching vertex since there are exactly two components in the forest $T^{[w]}-w$ such that 
each of the two components is rooted at a $k$-pre-branching vertex. The vertex $v$ is a $k$-branching vertex as it has a $k$-weakly-branching child $y$ and $J_{\mathrm{w}}^{k}(y)=2$.

In Figure 4.1(b), suppose that $v$ is the root and $L\left(z_{1}\right)=\left(k, \perp, s_{1} \sim s_{2}\right.$; $1,0 ; 0,0)$ and $L\left(z_{2}\right)=\left(k, \perp, s_{3} \sim s_{4} ; 1,0 ; 0,0\right)$. Then $x_{1}$ and $x_{2}$ are $k$-branching vertices with labels $\left(k, x_{1}, s_{1} \sim s_{2} ; 0,0 ; 0,0\right)$ and $\left(k, x_{2}, s_{3} \sim s_{4} ; 0,0 ; 0,0\right)$ respectively. As $w$ has two $k$-branching children $x_{1}$ and $x_{2}$, the label of $w$ is $(k+1, \perp, w ; 0,0 ; 0,0)$. Hence, $v$ is a $(k+1)$-pre-branching vertex with label $(k+1, \perp, v ; 0,0 ; 1,0)$.

Lemma 4.8. Let $T^{[u]}$ be a tree with $\mathrm{c}_{1}\left(T^{[u]}\right)=k$. Then $T^{[u]}$ has at most two utmost-k-pre-branching vertices. Moreover if $u$ is a $k$-pre-branching vertex then $T^{[u]}$ has a unique utmost-k-pre-branching vertex, if $u$ is a $k$-branching or $k$-weakly-branching vertex then $T^{[u]}$ has two utmost-k-pre-branching vertices.

Proof. Suppose that $T^{[u]}$ has at least three utmost-k-pre-branching descendants of $u$. We know an utmost- $k$-pre-branching descendant is a $k$-pre-branching vertex. So in the forest $T^{[u]}-N^{3}[u]$, three are at least three components with cop number $k$. From Theorem 3.5, we have $\mathrm{c}_{1}\left(T^{[u]}\right) \geq k+1$, which is a contradiction. So $T^{[u]}$ can not have three or more utmost- $k$-pre-branching descendants.

Suppose that $u$ is a $k$-pre-branching vertex and $T^{[u]}$ does not have any utmost- $k$-pre-branching descendant. As $u$ is a $k$-pre-branching vertex by definition $u$ is the utmost- $k$-pre-branching vertex and its unique.

Suppose that $u$ is a $k$-pre-branching vertex and $T^{[u]}$ has a $k$-pre-branching descendant. Then in the forest $T^{[u]}-u, \#_{\mathrm{pb}}^{k}\left(T^{[u]}-u\right)=1$. So there exist 
a $y^{t h}$ neighbour $z$ of $u$ such that for $y \geq 1$, $\#_{\mathrm{pb}}^{k}\left(T^{[u]}-N^{y-1}[u]\right)=1$, and $\#_{\mathrm{pb}}^{k}\left(T^{[u]}-N^{y}[u]\right)=0 . z$ is the utmost- $k$-pre-branching vertex and its unique.

Let $u$ is a $k$-weakly-branching vertex in $T^{[u]}$. From definition 4.1 we know $T^{[u]}$ has exactly two $k$-pre-branching vertices. We can see $T^{[u]}$ has exactly two utmost- $k$-pre-branching vertices.

Let $u$ is a $k$-branching vertex in $T^{[u]}$. From Definition 4.1 we know $T^{[u]}-$ $N^{2}[u]$ has exactly one component containing a $k$-weakly-branching vertex as the root. Similar to above we can say $T^{[u]}$ has exactly two utmost- $k$-prebranching vertices.

Lemma 4.9. Let $T^{[v]}$ be a tree where

$$
L(v)=\left(t_{m}^{v}, x_{m}^{v}, P_{m}^{v} ; \ldots ; t_{1}^{v}, \perp, P_{1}^{v} ; I_{\mathrm{wb}}^{t_{1}^{v}}(v), J_{\mathrm{w}}^{t_{1}^{v}}(v) ; I_{\mathrm{pb}}^{t_{1}^{v}}(v), J^{t_{1}^{v}}(v)\right)
$$

Then $T_{m}^{[v]}=T^{[v]}$ and for $\left.m>i \geq 2, T_{i}^{[v]}=T^{[v]}-\bigcup_{j=i+1}^{m} V\left(T^{\left[x_{j}^{v}\right]}\right)\right)$ each subtree contains an avenue.

Proof. For $2 \leq i \leq m, x_{i}^{v}$ is a $t_{i}^{v}$-branching vertex in $T_{i}^{[v]}$. From the Definition 4.1 we know the forest $T_{i}^{[v]}-N^{2}\left[x_{i}^{v}\right]$ has exactly one component whose root say $w_{i}$ is a $t_{i}^{v}$-weakly-branching vertex in that component. So, $T_{i}^{[v]}-N^{3}\left[w_{i}\right]$ has two components with cop number $t_{i}^{v}$ such that the path between them contains $w_{i}$. According to Definition 3.6, $w_{i}$ is an avenue vertex in $T_{i}^{[v]}$. So, $T_{i}^{[v]}$ contains an avenue.

From Lemma 4.9 we have the following corollary, 
Corollary 4.10. Let $T^{[v]}$ be a tree where

$$
L(v)=\left(t_{m}^{v}, x_{m}^{v}, P_{m}^{v} ; \ldots ; t_{1}^{v}, \perp, P_{1}^{v} ; I_{\mathrm{wb}}^{t_{1}^{v}}(v), J_{\mathrm{w}}^{t_{1}^{v}}(v) ; I_{\mathrm{pb}}^{t_{1}^{v}}(v), J^{t_{1}^{v}}(v)\right) .
$$

Then the two utmost-k-pre-branching vertices in $T_{m}^{[v]}=T^{[v]}$ and for $2 \leq$ $i<m$, the two utmost-k-pre-branching vertices in each subtree $T_{i}^{[v]}=T^{[v]}-$ $\left.\bigcup_{j=i+1}^{m} V\left(T^{\left[x_{j}^{v}\right]}\right)\right)$ are two pre-avenue vertices in that subtree.

Definition 4.11. Let $T^{[u]}$ be a tree with root $u$ whose children are $v_{1}, \ldots, v_{d}$. Suppose $c_{1}^{*}\left(T^{[u]}-u\right)=k \geq 1$. The counters $\#_{\mathrm{pb}}^{k}\left(T^{[u]}-u\right), \#_{\mathrm{wb}}^{k}\left(T^{[u]}-u\right)$, $\#_{\mathrm{c}}^{k}\left(T^{[u]}-u\right), h^{k}\left(T^{[u]}-u\right)$ and $h_{\mathrm{w}}^{k}\left(T^{[u]}-u\right)$ are defined as follows:

$$
\begin{gathered}
\#_{\mathrm{pb}}^{k}\left(T^{[u]}-u\right)=\sum_{j=1}^{d} I_{\mathrm{pb}}^{k}\left(v_{j}\right), \\
\#_{\mathrm{wb}}^{k}\left(T^{[u]}-u\right)=\sum_{j=1}^{d} I_{\mathrm{wb}}^{k}\left(v_{j}\right), \\
\#_{\mathrm{c}}^{k}\left(T^{[u]}-u\right)=\mid\left\{j \mid \mathrm{c}_{1}\left(T^{\left[v_{j}\right]}\right)=k \text { for } j \in\{1, \ldots, d\}\right\} \mid, \\
h^{k}\left(T^{[u]}-u\right)=\max \left\{J^{k}\left(v_{j}\right) \mid j \in\{1, \ldots, d\}\right\}, \\
h_{\mathrm{w}}^{k}\left(T^{[u]}-u\right)=\max \left\{J_{\mathrm{w}}^{k}\left(v_{j}\right) \mid j \in\{1, \ldots, d\}\right\} .
\end{gathered}
$$

\subsection{Algorithm for Computing $c_{1}(T)$}

The labels in Definition 4.6 have the following properties.

Theorem 4.12. Let $T^{[u]}$ be a tree with root $u$ whose children are $v_{1}, \ldots, v_{d}$. 
Suppose that $c_{1}^{*}\left(T^{[u]}-u\right)=k \geq 1$ and for $1 \leq j \leq d$,

$$
L_{T^{\left[v_{j}\right]}}\left(v_{j}\right)=\left(t^{v_{j}}, \perp, P^{v_{j}} ; I_{\mathrm{wb}}^{t^{v_{j}}}\left(v_{j}\right), J_{\mathrm{w}}^{t^{v_{j}}}\left(v_{j}\right) ; I_{\mathrm{pb}}^{t^{v_{j}}}\left(v_{j}\right), J^{t^{v_{j}}}\left(v_{j}\right)\right) .
$$

Then the label $L_{T^{[u]}}(u)$ must be of the form $\left(t^{u}, x^{u}, P^{u}, I_{\mathrm{wb}}^{t^{u}}(u), J_{\mathrm{w}}^{t^{u}}(u) ; I_{\mathrm{pb}}^{t^{u}}(u), J^{t^{u}}(u)\right)$ which can be determined as follows:

(1) If $\#_{\mathrm{wb}}^{k}\left(T^{[u]}-u\right)>1$, then $L_{T^{[u]}}(u)=(k+1, \perp, u ; 0,0 ; 0,0)$.

(2) If $\#_{\mathrm{wb}}^{k}\left(T^{[u]}-u\right)=1$ and $\#_{\mathrm{pb}}^{k}\left(T^{[u]}-u\right) \geq 1$, then $L_{T^{[u]}}(u)=(k+1, \perp$ $, u ; 0,0 ; 0,0)$.

(3) If $\#_{\mathrm{wb}}^{k}\left(T^{[u]}-u\right)=1, \#_{\mathrm{pb}}^{k}\left(T^{[u]}-u\right)=0$ and $\#_{\mathrm{c}}^{k}\left(T^{[u]}-u\right) \geq 2$, then $/^{*}$ Suppose $v_{1}$ is the k-weakly-branching child of $u$. */

(3.1) if $h_{\mathrm{w}}^{k}\left(T^{[u]}-u\right)=2$, then $L_{T^{[u]}}(u)=(k+1, \perp, u ; 0,0 ; 0,0)$.

(3.2) if $h_{\mathrm{w}}^{k}\left(T^{[u]}-u\right)=1$ and $h^{k}\left(T^{[u]}-u\right) \geq 1$, then $L_{T^{[u]}}(u)=(k+1, \perp, u$; $0,0 ; 0,0)$.

(3.3) if $h_{\mathrm{w}}^{k}\left(T^{[u]}-u\right)=1$ and $h^{k}\left(T^{[u]}-u\right)=0$, then $L_{T^{[u]}}(u)=\left(k, \perp, P^{v_{1}}\right.$; $1,2 ; 0,0)$.

(3.4) if $h_{\mathrm{w}}^{k}\left(T^{[u]}-u\right)=0$ and $h^{k}\left(T^{[u]}-u\right)=2$, then $L_{T^{[u]}}(u)=(k+1, \perp, u$; $0,0 ; 0,0)$.

(3.5) if $h_{\mathrm{w}}^{k}\left(T^{[u]}-u\right)=0$ and $h^{k}\left(T^{[u]}-u\right) \leq 1$, then $L_{T^{[u]}}(u)=\left(k, \perp, P^{v_{1}}\right.$; $1,1 ; 0,0)$.

(4) If $\#_{\mathrm{wb}}^{k}\left(T^{[u]}-u\right)=1$ and $\#_{\mathrm{c}}^{k}\left(T^{[u]}-u\right)=1$, then /* Suppose $v_{1}$ is the k-weakly-branching child of u. */ 
(4.1) if $h_{\mathrm{w}}^{k}\left(T^{[u]}-u\right)=2$, then $L_{T^{[u]}}(u)=\left(k, u, P^{v_{1}} ; 0,0 ; 0,0\right)$.

(4.2) if $h_{\mathrm{w}}^{k}\left(T^{[u]}-u\right)=1$, then $L_{T^{[u]}}(u)=\left(k, \perp, P^{v_{1}} ; 1,2 ; 0,0\right)$.

(4.3) if $h_{\mathrm{w}}^{k}\left(T^{[u]}-u\right)=0$, then $L_{T^{[u]}}(u)=\left(k, \perp, P^{v_{1}} ; 1,1 ; 0,0\right)$.

(5) If $\#_{\mathrm{wb}}^{k}\left(T^{[u]}-u\right)=0$, then

(5.1) if $\#_{\mathrm{pb}}^{k}\left(T^{[u]}-u\right) \geq 3$, then $L_{T^{[u]}}(u)=(k+1, \perp, u ; 0,0 ; 0,0)$.

if $\#_{\mathrm{pb}}^{k}\left(T^{[u]}-u\right)=2$, then $L_{T^{[u]}}(u)=\left(k, \perp, z_{1} \sim z_{2} ; 1,0 ; 0,0\right)$.

${ }^{*} z_{1}$ and $z_{2}$ are two utmost-k-pre-branching vertices in $T^{[u]} . * /$

(5.3) if $\#_{\mathrm{pb}}^{k}\left(T^{[u]}-u\right)=1$, then $L_{T^{[u]}}(u)=\left(k, \perp, z_{1} \sim u ; 0,0 ; 1,0\right)$.

${ }^{*} z_{1}$ is the utmost-k-pre-branching vertex in $T^{[u]} * /$

(5.4) if $\#_{\mathrm{pb}}^{k}\left(T^{[u]}-u\right)=0$, then

(5.4.1) if $h^{k}\left(T^{[u]}-u\right)=2$, then $L_{T^{[u]}}(u)=(k, \perp, u ; 0,0 ; 1,0)$.

(5.4.2) if $h^{k}\left(T^{[u]}-u\right)=1$, then $L_{T^{[u]}}(u)=(k, \perp, u ; 0,0 ; 0,2)$.

(5.4.3) if $h^{k}\left(T^{[u]}-u\right)=0$, then $L_{T^{[u]}}(u)=(k, \perp, u ; 0,0 ; 0,1)$.

Proof. (1) Since $\#_{\mathrm{wb}}^{k}\left(T^{[u]}-u\right)=\sum_{j=1}^{d} I_{\mathrm{wb}}^{k}\left(v_{j}\right) \geq 2$, we can suppose that $I_{\mathrm{wb}}^{k}\left(v_{1}\right)=I_{\mathrm{wb}}^{k}\left(v_{2}\right)=1$. For each $i=1,2$, from Definition 4.1. $T^{\left[v_{i}\right]}-v_{i}$, $T^{\left[v_{i}\right]}-N\left[v_{i}\right]$, or $T^{\left[v_{i}\right]}-N^{2}\left[v_{i}\right]$ has exactly two components whose root is a $k$ pre-branching vertex in the component. Since each $L_{T^{\left[v_{j}\right]}}\left(v_{j}\right)$ contains $\perp$ (see Equ. (4.2), we know that $u$ does not have a descendant that is a $k$-branching vertex. Thus, in the forest $T^{[u]}-N^{3}[u]$, there are at least four components with cop number $k$. It follows from Theorem 3.5 that $\mathrm{c}_{1}\left(T^{[u]}\right) \geq k+1$. Since $c_{1}^{*}\left(T^{[u]}-u\right)=k \geq 1$, we can clear $T^{[u]}$ by stationing one cop on $u$ and using 
the other $k$ cops to clear each component with cop number at most $k$. Thus $t^{u}=\mathrm{c}_{1}\left(T^{[u]}\right)=k+1$. Since $u$ and all its descendants are not $k$-branching, we have $x^{u}=\perp$. As $c_{1}\left(T^{[u]}\right)=k+1$ and all components in $T^{[u]}-N^{3}[u]$ has cop number at most $k, u$ is a hub in $T^{[u]}$, and by Definition 4.5 the hub $u$ is a road in $T^{[u]}$. Note that neither $u$ is $(k+1)$-weakly-branching nor it has a $(k+1)$ weakly-branching descendant. So $I_{\mathrm{wb}}^{k+1}(u)=0$ and $J_{\mathrm{w}}^{k+1}(u)=0$. Similarly, we have $I_{\mathrm{pb}}^{k+1}(u)=0$ and $J^{k+1}(u)=0$. Therefore $L_{T^{[u]}}(u)=(k+1, \perp, u ; 0,0 ; 0,0)$.

(2) Since $\#_{\mathrm{wb}}^{k}\left(T^{[u]}-u\right)=1$ and $\#_{\mathrm{pb}}^{k}\left(T^{[u]}-u\right) \geq 1$, in the forest $T^{[u]}-u$, there is exactly one component whose root is a $k$-weakly-branching vertex and at least one component whose root is a $k$-pre-branching vertex. W.l.o.g., suppose that $v_{1}$ is the $k$-weakly-branching vertex in $T^{\left[v_{1}\right]}$ and $v_{2}$ is a $k$-prebranching child of $u$. Thus, in the forest $T^{[u]}-N^{3}[u]$, there are at least three components with cop number $k$. According to Theorem $3.5, c_{1}\left(T^{[u]}\right) \geq k+1$. We can clear $T^{[u]}$ with $k+1$ cops by utilizing Hub-Strategy. Similar to case (1) we can prove $x^{u}=\perp, P^{u}=u, I_{\mathrm{wb}}^{k+1}(u)=0, J_{\mathrm{w}}^{k+1}(u)=0, I_{\mathrm{pb}}^{k+1}(u)=0$ and $J^{k+1}(u)=0$. Therefore $L_{T^{[u]}}(u)=(k+1, \perp, u ; 0,0 ; 0,0)$.

(3) Since $\#_{\mathrm{wb}}^{k}\left(T^{[u]}-u\right)=1$ and $\#_{\mathrm{c}}^{k}\left(T^{[u]}-u\right) \geq 2$, in the forest $T^{[u]}-u$, there is exactly one component whose root is a $k$-weakly-branching vertex. W.l.o.g., suppose that $v_{1}$ is the $k$-weakly-branching vertex in $T^{\left[v_{1}\right]}$ and $v_{2}$ is a child of $u$ with $\mathrm{c}_{1}\left(T^{\left[v_{2}\right]}\right)=k$.

$(3.1)$ If $h_{\mathrm{w}}^{k}\left(T^{[u]}-u\right)=2$, from Definitions 4.3 and 4.11 , we know $J_{\mathrm{w}}^{k}\left(v_{1}\right)=2$ and $c_{1}^{*}\left(T^{\left[v_{1}\right]}-N\left[v_{1}\right]\right)=k$, and further, there is exactly one component in the forest $T^{\left[v_{1}\right]}-N\left[v_{1}\right]$ such that the root of this component is a $k$-weakly- 
branching vertex in the component. Let $w$ be this $k$-weakly-branching vertex. From Definition 4.1, we know $T^{[w]}-N^{3}[w]$ has at least two components with cop number $k$. Furthermore, since $\mathrm{c}_{1}\left(T^{\left[v_{2}\right]}\right)=k, T^{[u]}-N^{3}[w]$ has at least three components with cop number $k$. From Theorem 3.5, we have $\mathrm{c}_{1}\left(T^{[u]}\right) \geq k+1$. Note that we can use $k+1$ cops to clear $T^{[u]}$ by Hub-Strategy. Thus $t^{u}=$ $\mathrm{c}_{1}\left(T^{[u]}\right)=k+1$. Similar to Case (1), we have $x^{u}=\perp, P^{u}=u$, and $I_{\mathrm{wb}}^{k+1}(u)=$ $J_{\mathrm{w}}^{k+1}(u)=I_{\mathrm{pb}}^{k+1}(u)=J^{k+1}(u)=0$. So, $L_{T^{[u]}}(u)=(k+1, \perp, u ; 0,0 ; 0,0)$.

(3.2) If $h_{\mathrm{w}}^{k}\left(T^{[u]}-u\right)=1$ and $h^{k}\left(T^{[u]}-u\right) \geq 1$, from Definitions 4.3 and 4.11 , we know $J_{\mathrm{w}}^{k}\left(v_{1}\right)=1$ and $c_{1}^{*}\left(T^{\left[v_{1}\right]}-v_{1}\right)=k$. So there is exactly one component in the forest $T^{\left[v_{1}\right]}-v_{1}$ such that the root of this component is a $k$-weaklybranching vertex in the component. Let $w$ be this $k$-weakly-branching vertex (see Figure 4.2). From Definition 4.1, we know $T^{[w]}-N^{3}[w]$ has at least two components with cop number $k$. Since $\mathrm{c}_{1}\left(T^{\left[v_{2}\right]}\right)=k$ and $h^{k}\left(T^{[u]}-u\right) \geq 1$, we know that $T^{[u]}-N^{3}[w]$ has at least three components with cop number $k$. Thus $\mathrm{c}_{1}\left(T^{[u]}\right) \geq k+1$. Similar to Case $(3.1)$, we have $L_{T^{[u]}}(u)=(k+1, \perp, u ; 0,0 ; 0,0)$.

(3.3) If $h_{\mathrm{w}}^{k}\left(T^{[u]}-u\right)=1$ and $h^{k}\left(T^{[u]}-u\right)=0$, similar to case (3.2), there is exactly one component in the forest $T^{\left[v_{1}\right]}-v_{1}$ such that the root $w$ of this component is a $k$-weakly-branching vertex in $T^{[w]}$ (see Figure 4.3 ). So $T^{[w]}-N^{3}[w]$ has two components with cop number $k$. Since $c_{1}\left(T^{\left[v_{2}\right]}\right)=k$ and $h^{k}\left(T^{[u]}-u\right)=0$, we have $\#_{\mathrm{c}}^{k}\left(T^{\left[v_{2}\right]}-v_{2}\right)=k-1$. Note that $\operatorname{dist}\left(w, v_{2}\right)=3$, which implies that $T^{[u]}-N^{3}[w]$ has exactly two components with cop number $k$. So there is an avenue in $T^{[u]}$ that contains $w$. Note that $L_{T^{[w]}}(w)=(k, \perp$ , $\left.z_{1} \sim z_{2} ; 1,0 ; 0,0\right)$. By Avenue-Strategy, we can use $k$ cops to clear $T^{[u]}$. So $t^{u}=\mathrm{c}_{1}\left(T^{[u]}\right)=k$. Since there is no $k$-branching vertex in $T^{[u]}$, it is easy 


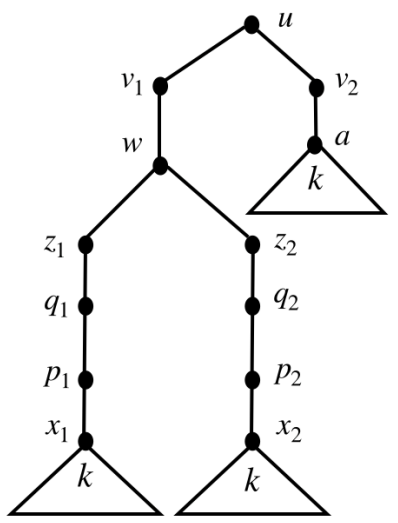

$$
\begin{aligned}
& L(u)=(k+1, \perp, u ; 0,0 ; 0,0) \\
& L\left(v_{2}\right)=\left(k, \perp, v_{2} ; 0,0 ; 0,1\right) \\
& L\left(v_{1}\right)=\left(k, \perp, z_{1} \sim z_{2} ; 1,1 ; 0,0\right) \\
& L(a)=(k, \perp, a ; 0,0 ; 0,0) \\
& L(w)=\left(k, \perp, z_{1} \sim z_{2} ; 1,0 ; 0,0\right) \\
& L\left(z_{2}\right)=\left(k, \perp, z_{2} ; 0,0 ; 1,0\right) \\
& L\left(z_{1}\right)=\left(k, \perp, z_{1} ; 0,0 ; 1,0\right) \\
& L\left(q_{2}\right)=\left(k, \perp, q_{2} ; 0,0 ; 0,2\right) \\
& L\left(q_{1}\right)=\left(k, \perp, q_{1} ; 0,0 ; 0,2\right) \\
& L\left(p_{2}\right)=\left(k, \perp, p_{2} ; 0,0 ; 0,1\right) \\
& L\left(p_{1}\right)=\left(k, \perp, p_{1} ; 0,0 ; 0,1\right) \\
& L\left(x_{2}\right)=\left(k, \perp, x_{2} ; 0,0 ; 0,0\right) \\
& L\left(x_{1}\right)=\left(k, \perp, x_{1} ; 0,0 ; 0,0\right)
\end{aligned}
$$

Figure 4.2: A tree $T^{[u]}$ with $c_{1}\left(T^{[u]}\right)=k+1$, where $L_{T^{\left[v_{1}\right]}}\left(v_{1}\right)=\left(k, \perp, z_{1} \sim\right.$ $\left.z_{2} ; 1,1 ; 0,0\right)$ and $L_{T^{\left[v_{2}\right]}}\left(v_{2}\right)=\left(k, \perp, v_{2} ; 0,0 ; 0,1\right)$.

to see that $x^{u}=\perp$. Since all components in $T^{[u]}-N^{3}[u]$ with cop number $k$ are the components in $T^{\left[v_{1}\right]}-N^{3}\left[v_{1}\right]$, the road in $T^{\left[v_{1}\right]}$ is also the road in $T^{[u]}$. Hence $P^{u}=P^{v_{1}}$. Since $\#_{\mathrm{wb}}^{k}\left(T^{[u]}-u\right)=1$ and $h_{\mathrm{w}}^{k}\left(T^{[u]}-u\right)=1$, we have $I_{\mathrm{wb}}^{k}(u)=1, J_{\mathrm{w}}^{k}(u)=2, I_{\mathrm{pb}}^{k}(u)=0$ and $J^{k}(u)=0$. Therefore $L_{T^{[u]}}(u)=(k, \perp$ ,$\left.P^{v_{1}} ; 1,2 ; 0,0\right)$.

$$
\begin{aligned}
& L(u)=\left(k, \perp, z_{1} \sim z_{2} ; 1,2 ; 0,0\right) \\
& L\left(v_{1}\right)=\left(k, \perp, z_{1} \sim z_{2} ; 1,1 ; 0,0\right) \\
& L\left(v_{2}\right)=\left(k, \perp, v_{2} ; 0,0 ; 0,0\right) \\
& L(w)=\left(k, \perp, z_{1} \sim z_{2} ; 1,0 ; 0,0\right) \\
& L\left(z_{1}\right)=\left(k, \perp, z_{1} ; 0,0 ; 1,0\right) \\
& L\left(q_{1}\right)=\left(k, \perp, q_{1} ; 0,0 ; 0,2\right) \\
& L\left(p_{1}\right)=\left(k, \perp, p_{1} ; 0,0 ; 0,1\right) \\
& L\left(x_{1}\right)=\left(k, \perp, x_{1} ; 0,0 ; 0,0\right)
\end{aligned}
$$

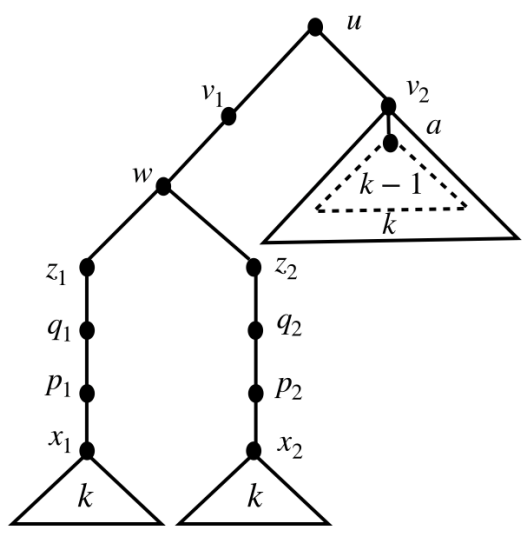

$$
\begin{aligned}
& L\left(z_{2}\right)=\left(k, \perp, z_{2} ; 0,0 ; 1,0\right) \\
& L\left(q_{2}\right)=\left(k, \perp, q_{2} ; 0,0 ; 0,2\right) \\
& L\left(p_{2}\right)=\left(k, \perp, p_{2} ; 0,0 ; 0,1\right) \\
& L\left(x_{2}\right)=\left(k, \perp, x_{2} ; 0,0 ; 0,0\right)
\end{aligned}
$$

Figure 4.3: A tree $T^{[u]}$ with $c_{1}\left(T^{[u]}\right)=k$, where $L_{T^{\left[v_{1}\right]}}\left(v_{1}\right)=\left(k, \perp, z_{1} \sim\right.$ $\left.z_{2} ; 1,1 ; 0,0\right)$ and $L_{T^{\left[v_{2}\right]}}\left(v_{2}\right)=\left(k, \perp, v_{2} ; 0,0 ; 0,0\right)$. 
(3.4) Similar to Case (3.2), we have $L_{T^{[u]}}(u)=(k+1, \perp, u ; 0,0 ; 0,0)$.

(3.5) Similar to Case (3.3), we can show $L_{T^{[u]}}(u)=\left(k, \perp, P^{v_{1}} ; 1,1 ; 0,0\right)$.

(4.1) Since $\#_{\mathrm{wb}}^{k}\left(T^{[u]}-u\right)=1, \#_{\mathrm{c}}^{k}\left(T^{[u]}-u\right)=1$ and $h_{\mathrm{w}}^{k}\left(T^{[u]}-u\right)=2$, we can suppose that $I_{\mathrm{wb}}^{k}\left(v_{1}\right)=1$. Then $J_{\mathrm{w}}^{k}\left(v_{1}\right)=2, c_{1}^{*}\left(T^{\left[v_{1}\right]}-N\left[v_{1}\right]\right)=k$ and there is exactly one component in the forest $T^{\left[v_{1}\right]}-N\left[v_{1}\right]$ such that the root say $w$, is a $k$-weakly-branching vertex in that component. So $T^{[w]}-N^{3}[w]$ has exactly two components with cop number $k$. As $\#_{c}^{k}\left(T^{[u]}-u\right)=1$, the forest $T^{[u]}-N^{3}[w]$ has only two components with cop number $k$. Similar to Case (3.3), we can clear $T^{[u]}$ with $k$ cops. Thus $t^{u}=k$. Since $c_{1}^{*}\left(T^{[u]}-N^{2}[u]\right)=k$ and there is exactly one component in the forest $T^{[u]}-N^{2}[u]$ whose root $w$ is a $k$-weakly-branching vertex, we have $x^{u}=u$. Similar to Case (3.3), $P^{u}=P^{v_{1}}$. From the structure of $T^{[u]}$, we have $I_{\mathrm{wb}}^{k}(u)=J_{\mathrm{w}}^{k}(u)=I_{\mathrm{pb}}^{k}(u)=J^{k}(u)=0$. Thus $L_{T^{[u]}}(u)=\left(k, u, P^{v_{1}} ; 0,0 ; 0,0\right)$.

(4.2) Similar to Case (3.3), we have $L_{T^{[u]}}(u)=\left(k, \perp, P^{v_{1}} ; 1,2 ; 0,0\right)$.

(4.3) Similar to Case (3.3), we can show $L_{T^{[u]}}(u)=\left(k, \perp, P^{v_{1}} ; 1,1 ; 0,0\right)$.

(5.1) Suppose that $\#_{\mathrm{wb}}^{k}\left(T^{[u]}-u\right)=0$ and $\#_{\mathrm{pb}}^{k}\left(T^{[u]}-u\right) \geq 3$. W.l.o.g., suppose that $v_{1}, v_{2}$ and $v_{3}$ are $k$-pre-branching vertices in $T^{\left[v_{1}\right]}, T^{\left[v_{2}\right]}$ and $T^{\left[v_{3}\right]}$, respectively (see Figure 4.4). For each $i=1,2,3$, from Definition 4.1, we know $c_{1}^{*}\left(T^{\left[v_{i}\right]}-N^{2}\left[v_{i}\right]\right)=k$. So in the forest $T^{[u]}-N^{3}[u]$, there are at least three components with cop number $k$. It follows from Theorem 3.5 that $\mathrm{c}_{1}\left(T^{[u]}\right) \geq$ $k+1$. We can clear $T^{[u]}$ by using Hub-Strategy. So $t^{u}=\mathrm{c}_{1}\left(T^{[u]}\right)=k+1$. It is easy to see that $x^{u}=\perp, P^{u}=u$, and $I_{\mathrm{wb}}^{k}(u)=J_{\mathrm{w}}^{k}(u)=I_{\mathrm{pb}}^{k}(u)=J^{k}(u)=0$. Thus $L_{T^{[u]}}(u)=(k+1, \perp, u ; 0,0 ; 0,0)$. 


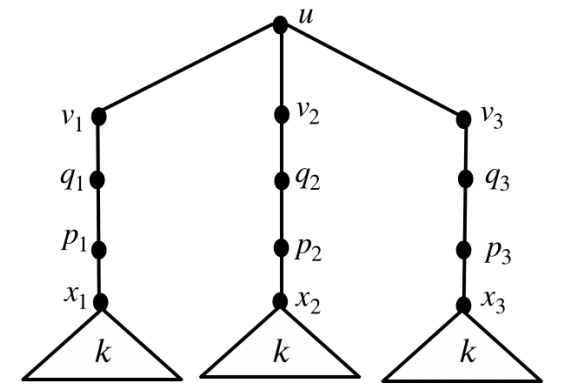

$$
\begin{aligned}
& L(u)=(k+1, \perp, u ; 0,0 ; 0,0) \\
& L\left(v_{i}\right)=\left(k, \perp, v_{i} ; 0,0 ; 1,0\right) ; i \in\{1,2,3\} \\
& L\left(q_{i}\right)=\left(k, \perp, q_{i} ; 0,0 ; 0,2\right) ; i \in\{1,2,3\} \\
& L\left(p_{i}\right)=\left(k, \perp, p_{i} ; 0,0 ; 0,1\right) ; i \in\{1,2,3\} \\
& L\left(x_{i}\right)=\left(k, \perp, x_{i} ; 0,0 ; 0,0\right) ; i \in\{1,2,3\}
\end{aligned}
$$

Figure 4.4: A tree $T^{[u]}$ with $\mathrm{c}_{1}\left(T^{[u]}\right)=k$, where $v_{1}, v_{2}$ and $v_{3}$ are three $k$-prebranching children of $u$.

(5.2) Suppose that $\#_{\mathrm{wb}}^{k}\left(T^{[u]}-u\right)=0$ and $\#_{\mathrm{pb}}^{k}\left(T^{[u]}-u\right)=2$. W.l.o.g., suppose that $v_{1}$ and $v_{2}$ are $k$-pre-branching vertices in $T^{\left[v_{1}\right]}$ and $T^{\left[v_{2}\right]}$, respectively (see Figure 4.5). So $T^{[u]}-N^{3}[u]$ has exactly two components with cop number $k$. Furthermore, there is an avenue in $T^{[u]}$ that contains $u$. By AvenueStrategy, we can use $k$ cops to clear $T^{[u]}$. So $t^{u}=\mathrm{c}_{1}\left(T^{[u]}\right)=k$. Since there is no $k$-branching vertex in $T^{[u]}$, it is easy to see that $x^{u}=\perp$. As $u$ is a $k$-weaklybranching vertex by Lemma $4.8, T^{[u]}$ has two utmost- $k$-pre-branching vertices. Let $z_{1}$ and $z_{2}$ be the two utmost- $k$-pre-branching vertices. Then $z_{1} \sim z_{2}$ is the road in $T^{[u]}$. It follows from Definitions 4.1 and 4.3 that $I_{\mathrm{wb}}^{k}(u)=1$ and $J_{\mathrm{w}}^{k}(u)=I_{\mathrm{pb}}^{t_{1}}(u)=J^{t_{1}}(u)=0$. Hence $L_{T^{[u]}}(u)=\left(k, \perp, z_{1} \sim z_{2} ; 1,0 ; 0,0\right)$.

(5.3) Suppose that $\#_{\mathrm{wb}}^{k}\left(T^{[u]}-u\right)=0, \#_{\mathrm{pb}}^{k}\left(T^{[u]}-u\right)=1$, then there is only one child of $u$, say $v_{1}$, which is a $k$-pre-branching vertex. So $T^{[u]}-N^{3}[u]$ has exactly one component with cop number $k$. It is not hard to see that $T^{[u]}$ can be cleared by $k$ cops. Thus $t^{u}=\mathrm{c}_{1}\left(T^{[u]}\right)=k$ and $x^{u}=\perp$. According to Lemma 4.8, $T^{[u]}$ has one utmost- $k$-pre-branching descendant of $u$. Suppose $z_{1}$ is the utmost- $k$-pre-branching vertex. Since $v_{1}$ is the only $k$-pre-branching 


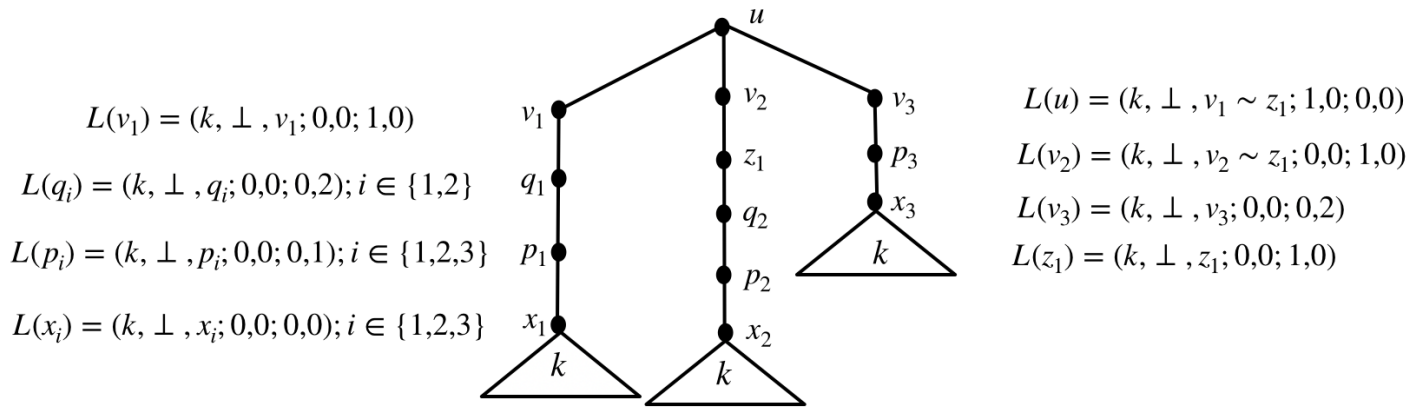

Figure 4.5: A tree $T^{[u]}$ with $\mathrm{c}_{1}\left(T^{[u]}\right)=k$, where $L_{T^{\left[v_{1}\right]}}\left(v_{1}\right)=\left(k, \perp, v_{1} ; 0,0 ; 1,0\right)$ and $L_{T^{\left[v_{2}\right]}}\left(v_{2}\right)=\left(k, \perp, v_{2} \sim z_{1} ; 0,0 ; 1,0\right)$ and $L_{T^{\left[v_{3}\right]}}\left(v_{3}\right)=\left(k, \perp, v_{3} ; 0,0 ; 0,2\right)$.

child of $u$, if $T^{[u]}$ contains an avenue then by Definition $4.5 z_{1}$ is an pre-avenue vertex and the path connecting $z_{1}$ and $u$ is the road containing the avenue. Figure 4.6 shows an example where the road $u \sim z_{1}$ in $T^{[u]}$ contains the only avenue vertex $y_{1}$. Similarly if $T^{[u]}$ contains a street then by Definition 4.5

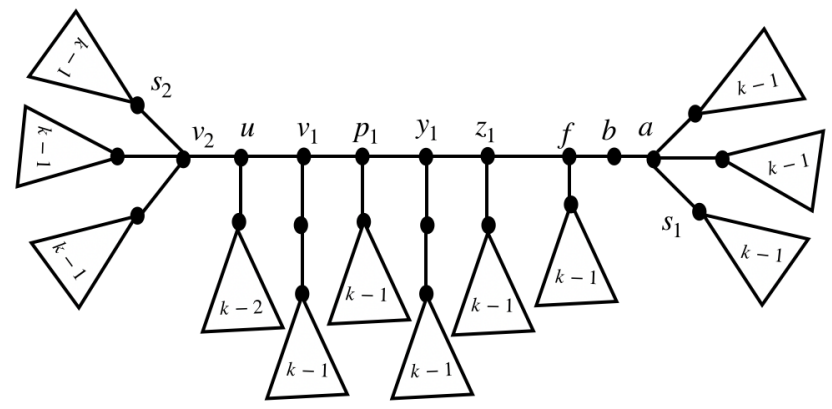

$$
\begin{aligned}
& L_{T}(u)=\left(k, \perp, u \sim z_{1} ; 0,0 ; 1,0\right) \\
& L_{T}\left(v_{1}\right)=\left(k, \perp, v_{1} \sim z_{1} ; 0,0 ; 1,0\right) \\
& L_{T}\left(v_{2}\right)=\left(k, \perp, v_{2} ; 0,0 ; 0,0\right) \\
& L_{T}\left(p_{1}\right)=\left(k, \perp, p_{1} \sim z_{1} ; 0,0 ; 1,0\right) \\
& L_{T}\left(y_{1}\right)=\left(k, \perp, y_{1} \sim z_{1} ; 0,0 ; 1,0\right) \\
& L_{T}\left(z_{1}\right)=\left(k, \perp, z_{1} ; 0,0 ; 1,0\right) \\
& L_{T}(f)=(k, \perp, f ; 0,0 ; 0,2) \\
& L_{T}(b)=(k, \perp, b ; 0,0 ; 0,1) \\
& L_{T}(a)=(k, \perp, a ; 0,0 ; 0,0)
\end{aligned}
$$

Figure 4.6: A tree $T^{[u]}$ with $\mathrm{c}_{1}\left(T^{[u]}\right)=k$, where $u \sim z_{1}$ is the road containing an avenue vertex $y_{1}$.

$z_{1} \sim u$ is the road containing the street vertices $z_{1}$ and the parent of $z_{1}$. See figure 4.7 .

If there is no avenue or street then by Definition $4.5 z_{1} \sim u$ is the road containing a hub $z_{1}$ in $T^{[u]}$. See figure 4.8 . Since there is no $k$-weakly-branching 


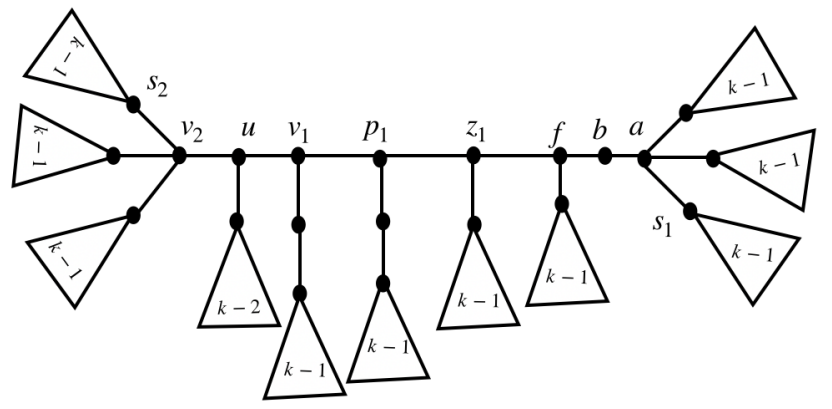

$$
\begin{aligned}
& L_{T}(u)=\left(k, \perp, u \sim z_{1} ; 0,0 ; 1,0\right) \\
& L_{T}\left(v_{1}\right)=\left(k, \perp, v_{1} \sim z_{1} ; 0,0 ; 1,0\right) \\
& L_{T}\left(v_{2}\right)=\left(k, \perp, v_{2} ; 0,0 ; 0,0\right) \\
& L_{T}\left(p_{1}\right)=\left(k, \perp, p_{1} \sim z_{1} ; 0,0 ; 1,0\right) \\
& L_{T}\left(z_{1}\right)=\left(k, \perp, z_{1} ; 0,0 ; 1,0\right) \\
& L_{T}(f)=(k, \perp, f ; 0,0 ; 0,2) \\
& L_{T}(b)=(k, \perp, b ; 0,0 ; 0,1) \\
& L_{T}(a)=(k, \perp, a ; 0,0 ; 0,0)
\end{aligned}
$$

Figure 4.7: A tree $T^{[u]}$ with $c_{1}\left(T^{[u]}\right)=k$, where $u \sim z_{1}$ is the road containing street vertices $z_{1}$ and $p_{1}$.

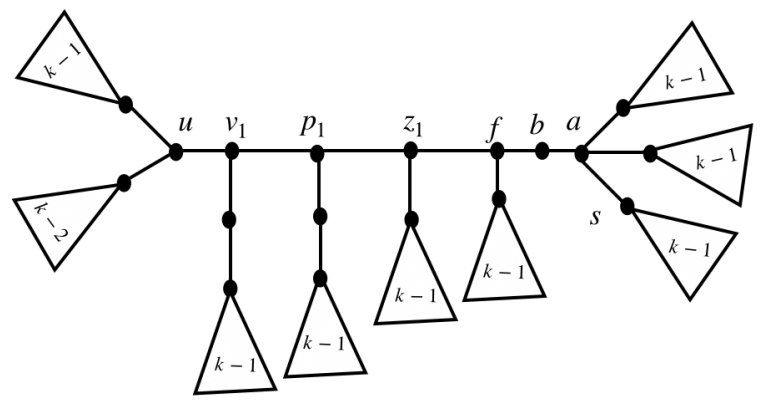

$$
\begin{aligned}
& L_{T}(u)=\left(k, \perp, u \sim z_{1} ; 0,0 ; 1,0\right) \\
& L_{T}\left(v_{1}\right)=\left(k, \perp, v_{1} \sim z_{1} ; 0,0 ; 1,0\right) \\
& L_{T}\left(p_{1}\right)=\left(k, \perp, p_{1} \sim z_{1} ; 0,0 ; 1,0\right) \\
& L_{T}\left(z_{1}\right)=\left(k, \perp, z_{1} ; 0,0 ; 1,0\right) \\
& L_{T}(f)=(k, \perp, f ; 0,0 ; 0,2) \\
& L_{T}(b)=(k, \perp, b ; 0,0 ; 0,1) \\
& L_{T}(a)=(k, \perp, a ; 0,0 ; 0,0)
\end{aligned}
$$

Figure 4.8: A tree $T^{[u]}$ with $\mathrm{c}_{1}\left(T^{[u]}\right)=k$ and with no street or avenue vertex, the road $u \sim z_{1}$ is the road containing a hub $z_{1}$.

vertex, we have $I_{\mathrm{wb}}^{k}(u)=J_{\mathrm{w}}^{k}(u)=0$. It follows from Definition 4.1 that $I_{\mathrm{pb}}^{k}(u)=1$. From Definition 4.3 , $J^{k}(u)=0$. Therefore $L_{T^{[u]}}(u)=(k, \perp, u \sim$ $\left.z_{1} ; 0,0 ; 1,0\right)$.

(5.4.1) Suppose that $\#_{\mathrm{wb}}^{k}\left(T^{[u]}-u\right)=0, \#_{\mathrm{pb}}^{k}\left(T^{[u]}-u\right)=0$ and $h^{k}\left(T^{[u]}-u\right)=$ 2. From Definitions 4.11 and 4.3, we have $t^{u}=k, x^{u}=\perp$, and $I_{\mathrm{wb}}^{k}(u)=$ $J_{\mathrm{w}}^{k}(u)=0$. Similar to Case $(5.3)$, we can show that $I_{\mathrm{pb}}^{k}(u)=1$ and $J^{k}(u)=0$. Since $T^{[u]}-N^{3}[u]$ has no components with cop number $k, u$ is a hub in $T^{[u]}$. So $u$ is a road in $T^{[u]}$. Hence $L_{T^{[u]}}(u)=(k, \perp, u ; 0,0 ; 1,0)$. 


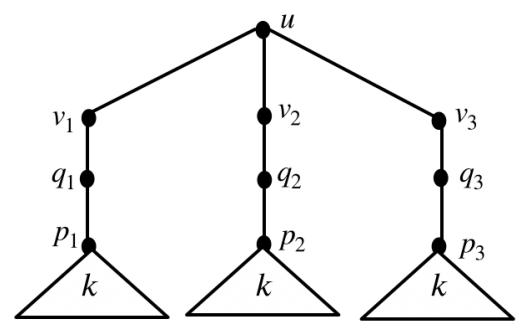

$$
\begin{aligned}
L(u) & =(k, \perp, u ; 0,0 ; 1,0) \\
L\left(v_{i}\right) & =\left(k, \perp, v_{i} ; 0,0 ; 0,2\right) ; i \in\{1,2,3\} \\
L\left(q_{i}\right) & =\left(k, \perp, q_{i} ; 0,0 ; 0,1\right) ; i \in\{1,2,3\} \\
L\left(p_{i}\right) & =\left(k, \perp, p_{i} ; 0,0 ; 0,0\right) ; i \in\{1,2,3\}
\end{aligned}
$$

Figure 4.9: A tree $T^{[u]}$ with $c_{1}\left(T^{[u]}\right)=k$ and a hub $u$ in $T^{[u]}$.

$(5.4 .2)$ Suppose that $\#_{\mathrm{wb}}^{k}\left(T^{[u]}-u\right)=0, \#_{\mathrm{pb}}^{k}\left(T^{[u]}-u\right)=0$ and $h^{k}\left(T^{[u]}-\right.$ $u)=1$. Similar to Case (5.4.1), we have $t^{u}=k, x^{u}=\perp, P^{u}=u$ and $I_{\mathrm{wb}}^{k}(u)=J_{\mathrm{w}}^{k}(u)=0$. From Definitions 4.1 and 4.3 we know that $I_{\mathrm{pb}}^{k}(u)=0$ and $J^{k}(u)=2$. So $L_{T^{[u]}}(u)=(k, \perp, u ; 0,0 ; 0,2)$.

(5.4.3) Similar to Case (5.4.2), we have $L_{T^{[u]}}(u)=(k, \perp, u ; 0,0 ; 0,1)$.

Algorithm 1 is a bottom-up approach for computing the labels of a tree. In this algorithm, we first assign labels to each vertex that has no children. Then for each vertex whose children have been labeled, we compute the label of this vertex using the rules proved in Theorems $4.12,4.13$ and 4.14 . Finally, the first component in the label of the root is the 1-visibility cop number of the tree and third component is the road. Recall that the label of $v$ in $T^{[v]}$ has the form below:

$$
L_{T^{[v]}}(v)=\left(t_{m}^{v}, x_{m}^{v}, P_{m}^{v} ; \ldots ; t_{1}^{v}, x_{1}^{v}, P_{1}^{v} ; I_{\mathrm{wb}}^{t_{1}^{v}}(v), J_{\mathrm{w}}^{t_{1}^{v}}(v) ; I_{\mathrm{pb}}^{t_{1}^{v}}(v), J^{t_{1}^{v}}(v)\right),
$$

where the first pair $\left(t_{m}^{v}, x_{m}^{v}, P_{m}^{v}\right)$ is an item associated with $T^{[v]}$, and each pair $\left(t_{i}^{v}, x_{i}^{v}, P_{i}^{v}\right), 1 \leq i<m$, is an item associated with subtree $T^{[v]}-\bigcup_{j=i+1}^{m} V\left(T^{\left[x_{j}^{v}\right]}\right)$. 
Note that $t_{i}^{v}$ is called the key of the item and $x_{i}^{v}$ and $P_{i}^{v}$ are its attributes.

Let $u$ be the root of the tree in Figure 4.10 which has five children $v_{1}, v_{2}, v_{3}$, $v_{4}$, and $v_{5}$. If we consider in Step 4 of Algorithm 1 the labels of $v_{1}, v_{2}, v_{3}, v_{4}$, and $v_{5}$ are those given in the Figure 4.10 then in Step $5, T_{1}$ should be the subtree inside dotted region. In Step 6, we get $L_{T_{1}^{[u]}}(u)=\left(4, \perp, v_{1} \sim v_{2} ; 1,0 ; 0,0\right)$. After executing Steps 7-11 and then going back to Step 3, the algorithm returns $L_{T[u]}(u)=\left(8, x_{2}, s_{3} \sim s_{4} ; 7, \perp, u ; 0,0 ; 0,0\right)$.

$$
\begin{aligned}
& L_{T}(u)=\left(8, x_{2}, s_{3} \sim s_{4} ; 7, \perp, u ; 0,0 ; 0,0\right) \\
& L_{T_{1}}(u)=\left(4, \perp, v_{1} \sim v_{2} ; 1,0 ; 0,0\right) \\
& L\left(v_{1}\right)=\left(4, \perp, v_{1} ; 0,0 ; 1,0\right) \\
& L\left(v_{2}\right)=\left(4, \perp, v_{2} ; 0,0 ; 1,0\right) \\
& L\left(v_{3}\right)=\left(5, x_{1}, s_{1} \sim s_{2} ; 2, \perp, v_{3} ; 0,0 ; 0,2\right) \\
& L\left(v_{4}\right)=\left(8, x_{2}, s_{3} \sim s_{4} ; 4, v_{4}, s_{5} \sim s_{6} ; 0,0 ; 0,0\right) \\
& L\left(v_{5}\right)=\left(6, x_{3}, s_{7} \sim s_{8} ; 2, v_{5}, s_{9} \sim s_{10} ; 0,0 ; 0,0\right)
\end{aligned}
$$

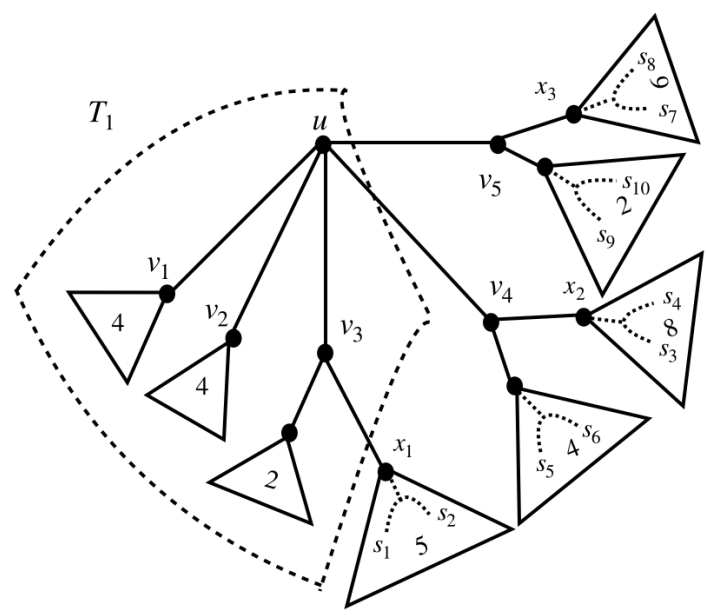

Figure 4.10: Computing the label of $u$ in $T^{[u]}$. 
Algorithm 1 Computing the labels of a tree with road information

Input: A tree $T$ with $n \geq 3$ vertices.

Output: The label of the root of $T$.

1: Pick a vertex of $T$ as its root.

2: Sort the vertices of $T$ to a list $u_{1}, \ldots, u_{n}$ such that every vertex is before its parent in the list. For each vertex $u$ that has no child, set its label as $(1, \perp, u ; 0,0 ; 0,0)$.

3: If the root $u_{n}$ has obtained a label $L_{T^{\left[u_{n}\right]}}\left(u_{n}\right)$, then output this label; otherwise, let $u$ be the first unlabeled vertex in the list currently. Run Steps 4 to 11 to compute $L_{T^{[u]}}(u)$.

4: Let $v_{j}, 1 \leq j \leq d$, be all children of $u$ with labels $L_{T^{\left[v_{j}\right]}}\left(v_{j}\right)$ in the form of Equ. (4.3). Let $I_{\perp}$ be the subset of children whose label contains $\perp$ and let $I_{b}$ be the subset of children whose label does not contain $\perp$.

5: Construct $T_{1}^{[u]}$, where $T_{1}^{[u]}=T^{[u]}-\bigcup_{y \in I_{b}} V\left(T^{\left[x_{1}^{y}\right]}\right)-\bigcup_{y \in I_{\perp}} V\left(T^{\left[x_{2}^{y}\right]}\right)$. For $v \in$ $V\left(T_{1}\right)-\{u\}, L_{T_{1}^{[v]}}(v)$ is obtained by deleting all components except the last seven components from $L_{T^{[v]}}(v)$.

6: If $T_{1}^{[u]}$ contains only one vertex $u$ then $L_{T_{1}^{[u]}}(u)=(1, \perp, u ; 0,0 ; 0,0)$, otherwise, compute $L_{T_{1}^{[u]}}(u)$ by Theorem 4.12. Let $k=\left|L_{T_{1}^{[u]}}(u)\right|$.

7: For $1 \leq j \leq d$, if $v_{j} \in I_{\perp}$, let $L_{j}$ be a list obtained from $L_{T^{\left[v_{j}\right]}}\left(v_{j}\right)$ by deleting the last seven components and items whose key is less than $k$; if $v_{j} \in I_{b}$, let $L_{j}$ be a list obtained from $L_{T^{\left[v_{j}\right]}}\left(v_{j}\right)$ by deleting the last four components and items whose key is less than $k$. Let $L_{d+1}$ be a list containing only the first item of $L_{T_{1}^{[u]}}(u)$.

8: If no key in $L_{1}, \ldots, L_{d}, L_{d+1}$ is repeated, then $L_{T^{[u]}}(u) \leftarrow L_{T_{1}^{[u]}}(u)$ and insert the items of $L_{1}, \ldots, L_{d}$ into $L_{T^{[u]}}(u)$. Go to Step 3 .

9: Find the largest repeated key $k^{*}$ in the lists $L_{1}, \ldots, L_{d}, L_{d+1}$.

10: Let $K=\left(k_{1}, \ldots, k_{\ell}\right)$ be a list containing the distinct keys from $L_{1}, \ldots, L_{d+1}$ satisfying that the keys in $K$ are decreasing and are greater than or equal to $k^{*}$.

11: Find the smallest index $h$ in $K$, where $1 \leq h \leq \ell$, such that $k_{h}=k_{h+1}+1=$ $\cdots=k_{\ell}+(\ell-h)$. Update $K \leftarrow\left(k_{1}, \ldots, k_{h-1}, k_{h}^{\prime}\right)$ where $k_{h}^{\prime}=k_{h}+1$. Create a list $X=\left(Q_{1}, \ldots, Q_{h-1}, Q_{h}\right)$, where $Q_{i}=\left(k_{i}, x_{i}, P_{i}\right), 1 \leq i \leq h-1$, is an item with key $k_{i}$ and attributes $x_{i}$ and $P_{i}$ (note that $x_{i}, 1 \leq i \leq h-1$, is a $k_{i}$-branching vertex in some subtree and $P_{i}$ is the road in that subtree) and $Q_{h}=\left(k_{h}^{\prime}, \perp ; u\right)$.Insert $(0,0 ; 0,0)$ at the end of $X$. Set $L_{T^{[u]}}(u) \leftarrow X$. Go to Step 3 . 


\subsection{Correctness and Time Complexity}

Theorem 4.13. Let $T^{[u]}$ be a rooted tree and $v_{1}, \ldots, v_{d}$ be the children of the root $u$. Let $\mathrm{c}_{1}\left(T^{\left[v_{j}\right]}\right) \leq k$, for $1 \leq i \leq d$. Suppose that $L_{T^{\left[v_{j}\right]}}\left(v_{j}\right)=$

$\begin{cases}\left(t_{1}^{v_{j}}, \perp, P_{1}^{v_{j}} ; I_{\mathrm{wb}}^{t_{1}^{v_{j}}}\left(v_{j}\right), J_{\mathrm{w}}^{t_{1}^{v_{j}}}\left(v_{j}\right) ; I_{\mathrm{pb}}^{t_{1}^{v_{j}}}\left(v_{j}\right), J^{t_{1}^{v_{j}}}\left(v_{j}\right)\right), & \text { if } 1 \leq j \leq d_{1}, \\ \left(t_{m_{j}}^{v_{j}}, x_{m_{j}}^{v_{j}}, P_{m_{j}}^{v_{j}} ; \ldots ; t_{1}^{v_{j}}, \perp, P_{1}^{v_{j}} ; I_{\mathrm{wb}}^{t_{1}^{v_{j}}}\left(v_{j}\right), J_{\mathrm{w}}^{t_{j}}\left(v_{j}\right) ; I_{\mathrm{pb}}^{t_{1}^{v_{j}}}\left(v_{j}\right), J^{t_{1}^{v_{j}}}\left(v_{j}\right)\right), & \text { if } d_{1}<j \leq d_{2}, \\ \left(t_{m_{j}}^{v_{j}}, x_{m_{j}}^{v_{j}}, P_{m_{j}}^{v_{j}} ; \ldots ; t_{1}^{v_{j}}, x_{1}^{v_{j}}, P_{1}^{v_{j}} ; 0,0 ; 0,0\right), & \text { if } d_{2}<j \leq d,\end{cases}$

where $x_{1}^{v_{j}}, d_{2}<j \leq d$, are $t_{1}^{v_{j}}$-branching vertices of $T^{[u]}$. Let $T_{1}^{[u]}=T^{[u]}-$ $\bigcup_{j=d_{1}+1}^{d_{2}} V\left(T^{\left[x_{2}^{v_{j}}\right]}\right)-\bigcup_{j=d_{2}+1}^{d} V\left(T^{\left[x_{1}^{v_{j}}\right]}\right)$ and $\mathrm{c}_{1}\left(T_{1}^{[u]}\right)=k$. If $t_{m_{j}}^{v_{j}}<k$ for each $j \in$ $\left\{d_{1}+1, \ldots, d\right\}$, then $\mathrm{c}_{1}\left(T^{[u]}\right)=k$ and $P_{T^{[u]}}=P_{T_{1}^{[u]}}$. If $t_{m_{j}}^{v_{j}}=k$ for at least one $j \in\left\{d_{1}+1, \ldots, d\right\}$, then $\mathrm{c}_{1}\left(T^{[u]}\right)=k+1$ and $P_{T^{[u]}}=u$.

Proof. For $j \in\left\{d_{1}+1, \ldots, d\right\}$, since $t_{m_{j}}^{v_{j}}<k$, we can use $k-1$ cops to clear $T^{\left[v_{j}\right]}$. Let $T_{1}^{[u]}=T^{[u]}-\bigcup_{j=d_{1}+1}^{d_{2}} V\left(T^{\left[x_{2}^{v_{j}}\right]}\right)-\bigcup_{j=d_{2}+1}^{d} V\left(T^{\left[x_{1}^{v_{j}}\right]}\right)$. Since $\mathrm{c}_{1}\left(T_{1}^{[u]}\right)=k$, we can clear tree $T_{1}^{[u]}$ with $k$ cops. We now give a cops' strategy to clear the whole tree $T^{[u]}$ with $k$ cops.

CASE 1. Let $u$ be a hub in $T_{1}^{[u]}$. So the road in $T_{1}^{[u]}$ is $u$. As $t_{m_{j}}^{v_{j}}<k$, $d_{1}+1 \leq j \leq d, T^{[u]}-N^{3}[u]$ does not have any components with cop number $k$. So $u$ is also a hub in $T^{[u]}$ as well as a road in $T^{[u]}$. To clear $T^{[u]}$, we first use $k$ cops to clear $T_{1}^{[u]}$, and then let one cop stay on $u$ and use $k-1$ cops to clear the subtrees $T^{\left[v_{j}\right]}, d_{1}+1 \leq j \leq d$.

CASE 2. Let $u$ be a street vertex in $T_{1}^{[u]}$ and $u \sim v$ be the street where $v$ is 
a child of $u$. As $t_{m_{j}}^{v_{j}}<k, d_{1}+1 \leq j \leq d$, all components in $T^{[u]}-N^{3}[u]$ (resp. $T^{[v]}-N^{3}[v]$ ) with cop number $k$ are the components in $T_{1}^{[u]}-N^{3}[u]$ (resp. $\left.T_{1}^{[v]}-N^{3}[v]\right)$. So $u$ and $v$ are also street vertices in $T^{[u]}$. Hence $u \sim v$ is the street in $T^{[u]}$. For clearing $T^{[u]}, k$ cops first clear the subtree $T^{\left[v_{1}\right]}$ rooted at $v_{1}$ using Hub-Strategy. After that all $k$ cops move to $u$ to clear the subtree $T^{[u]}-V\left(T^{\left[v_{1}\right]}\right)$ rooted at $u$ using HuB-STRATEGY.

CASE 3. Let $u$ is an avenue vertex and $z_{1} \sim z_{2}$ is the road in $T_{1}^{[u]}$ where $z_{1}$ and $z_{2}$ are two utmost- $k$-pre-branching vertices in $T_{1}^{[u]}$. As $t_{m_{j}}^{v_{j}}<k, d_{1}+1 \leq$ $j \leq d$, components in $T^{[u]}-N^{3}[u]$ containing $z_{1}$ and $z_{2}$ are the components in $T_{1}^{[u]}-N^{3}[u]$. So $z_{1}$ and $z_{2}$ are also two pre-avenue vertices in $T^{[u]}$. Hence $z_{1} \sim z_{2}$ is also the road in $T^{[u]}$. The $k$ cops can clear $T^{[u]}$ using AvenueSTRATEGY, during which when all cops move to the vertex $u$ on the avenue, $k-1$ cops can clear each subtree $T^{\left[v_{j}\right]}, d_{1}+1 \leq j \leq d$, attached to $u$, as $t_{m_{j}}^{v_{j}}<k$.

CASE 4. Suppose that $u$ is not a hub, a street vertex, or an avenue vertex in $T_{1}^{[u]}$. Assume that $T_{1}^{[u]}$ has an avenue $x_{1} \cdots x_{m}$. Let $x_{0}$ be the pre-avenue vertex adjacent to $x_{1}$ and let $x_{m+1}$ be the pre-avenue vertex adjacent to $x_{m}$. Since $u$ is not an avenue vertex in $T_{1}^{[u]}$, the avenue is contained in only one component of $T_{1}^{[u]}-u$. W.l.o.g., suppose that $T^{\left[v_{1}\right]}$ contains all $x_{i}, 0 \leq i \leq$ $m+1$. So the road in $T^{\left[v_{1}\right]}$ is the $\operatorname{road}$ in $T_{1}^{[u]}$. Since $\mathrm{c}_{1}\left(T_{1}^{[u]}\right)=k$ and $T^{\left[v_{1}\right]}$ contains the avenue of $T_{1}^{[u]}$, we know that $\mathrm{c}_{1}\left(T^{\left[v_{1}\right]}\right)=k$. Among the vertices in $\left\{x_{0}, x_{1}, \ldots, x_{m}, x_{m+1}\right\}$, let $x_{j}$ be the closest vertex to $u$ in $T^{[u]}$. We now modify the Avenue-Strategy for clearing $T_{1}^{[u]}$ to a strategy for clearing $T^{[u]}$. When we use the Avenue-Strategy to clear $T_{1}^{[u]}$, there is a moment at which all 
$k$ cops are located at $x_{j}$. While one cop protects $x_{j}$ by staying on it or its neighbour $x_{j}^{\prime}$ between $x_{j}$ and $u$, or visiting $x_{j}^{\prime}$ every other term, the other $k-1$ cops can clear all components $T_{1}^{\left[v_{j}\right]}, 2 \leq j \leq d_{1}$. Since $T_{1}^{\left[v_{j}\right]}$ is the same as components $T^{\left[v_{j}\right]}, 2 \leq j \leq d_{1}$, and $t_{m_{j}}^{v_{j}}<k, d_{1}+1 \leq j \leq d$, these $k-1$ cops can also clear components $T^{\left[v_{j}\right]}, d_{1}+1 \leq j \leq d$. Thus $k$ cops can clear the tree $T^{[u]}$. As $t_{m_{j}}^{v_{j}}<k, d_{1}+1 \leq j \leq d$, components in $T^{[u]}-N^{3}[u]$ containing the avenue are the components in $T_{1}^{[u]}-N^{3}[u]$. So $P_{T^{[u]}}=P_{T_{1}^{[u]}}$.

Similarly, we can show that if $T_{1}^{[u]}$ has a hub $x$ or a street vertex $x$ and $u$ is a vertex in some component of $T_{1}^{[u]}-x$, we can still use $k$ cops to clear $T^{[u]}$ and the path in $T_{1}^{[u]}$ is the path in $T^{[u]}$.

Now consider $t_{m_{j}}^{v_{j}}=k$ for at least one $j \in\left\{d_{1}+1, \ldots, d\right\}$. Then there exist a $k$-branching child of $u$ in $T^{[u]}$, and $T^{[u]}-N^{3}[u]$ has a $k$-weakly-branching vertex say $w_{1}$ in $T^{[u]}$. From Definitions 4.1, there are exactly two components in the forest $T^{\left[w_{1}\right]}-w_{1}$ such that the root of this component is a $k$-pre-branching vertex. Thus in the forest $T^{[u]}-N^{3}\left[w_{1}\right]$, there exist at least three components with cop number $k$. It follows from Theorem 3.5 that $c_{1}\left(T^{[u]}\right) \geq k+1$. We can clear $T^{[u]}$ by stationing one cop on $u$ and using the other $k$ cops to clear each component with cop number at most $k$. Thus $\mathrm{c}_{1}\left(T^{[u]}\right)=k+1$. As $T-N^{3}[u]$ does not have any component with cop number $k+1, u$ is a hub in $T^{[u]}$. Thus $u$ is a road in $T^{[u]}$.

From the above Theorem we know if $\mathrm{c}_{1}\left(T^{[u]}\right)=k$ then $P_{T^{[u]}}=P_{T_{1}^{[u]}}$. So if $P_{T_{1}^{[u]}}$ contains a $k$-branching vertex then $x_{1}^{u}=u$, otherwise $x_{1}^{u}=\perp$.

The following theorem is an extension of Theorem 3.5. 
Theorem 4.14. Let $T^{[u]}$ be a rooted tree and $v_{1}, \ldots, v_{d}$ be the children of the root $u$. Suppose that for $1 \leq j \leq d$,

$$
L_{T^{\left[v_{j}\right]}}\left(v_{j}\right)=\left(t_{m_{j}}^{v_{j}}, x_{m_{j}}^{v_{j}}, P_{m_{j}}^{v_{j}} ; \ldots ; t_{1}^{v_{j}}, x_{1}^{v_{j}}, P_{1}^{v_{j}} ; 0,0 ; 0,0\right)
$$

where $t_{m_{1}}^{v_{1}} \geq t_{m_{2}}^{v_{2}} \geq \cdots \geq t_{m_{d}}^{v_{d}}$ and $x_{1}^{v_{j}} \neq \perp$. Let $k=c_{1}^{*}\left(T^{[u]}-u\right)$. If $c_{1}\left(T^{\left[v_{1}\right]}\right)=$ $\mathrm{c}_{1}\left(T^{\left[v_{2}\right]}\right)=k$, then $L(u)=(k+1, \perp, u ; 0,0 ; 0,0)$.

Proof. Since $v_{1}$ and $v_{2}$ are two $k$-branching children of $u$ in $T^{[u]}$, each $T^{\left[v_{i}\right]}$, $i=1,2$, has a $k$-weakly-branching vertex $w_{i}, i=1,2$. From Definitions 4.1 , there are exactly two components in the forest $T^{\left[w_{1}\right]}-w_{1}$ such that the root of this component is a $k$-pre-branching vertex. Thus in the forest $T^{[u]}-N^{3}\left[w_{1}\right]$, there exist at least three components with cop number $k$. It follows from Theorem 3.5 that $\mathrm{c}_{1}\left(T^{[u]}\right) \geq k+1$. We can clear $T^{[u]}$ with $k+1$ cops in the following way: First one cop protects $w_{1}$ and the other $k$ cops clear all components in $T^{\left[w_{1}\right]}-w_{1}$; then all cops move along the path from $w_{1}$ to $w_{2}$ to clear all branches attached on the internal vertices of the path; finally, one cop stays on $w_{2}$ and the other $k$ cops clear all components in $T^{\left[w_{2}\right]}-w_{2}$. Therefore $\mathrm{c}_{1}\left(T^{[u]}\right)=k+1$.

As $T-N^{3}[u]$ does not have any component with cop number $k+1, u$ is a hub in $T^{[u]}$. According to Definition 4.5, $u$ is a road in $T^{[u]}$. Since $u$ and all its descendants are not $(k+1)$-branching, we have $x^{u}=\perp$. Note that neither $u$ is $(k+1)$-weakly-branching nor it has a $(k+1)$-weakly-branching descendant. So $I_{\mathrm{wb}}^{k+1}(u)=0$ and $J_{\mathrm{w}}^{k+1}(u)=0$. Similarly, we have $I_{\mathrm{pb}}^{k+1}(u)=0$ and $J^{k+1}(u)=0$. Hence $L(u)=(k+1, \perp, u ; 0,0 ; 0,0)$. 
We next show the correctness of Algorithm 1 .

Theorem 4.15. For a tree $T$ with at least three vertices, Algorithm 1 computes the label of the root of $T$.

Proof. First, arbitrarily select a vertex as the root of $T$, which induces the parent-child relation in the tree. Then the vertices of $T$ can be ordered such that each vertex is listed before its parent. Let $u_{1}, \ldots, u_{n}$ be such an ordered list of vertices. So $u_{n}$ is the root of $T$. If a vertex has no child, then from Definition 4.6, its label is $(1, \perp, u ; 0,0 ; 0,0)$. Let $u$ be a vertex in the list whose children's labels have been computed. Let $v_{1}, \ldots, v_{d}$ be all children of $u$ with labels in the form of Euq. 4.3. . Let $T_{1}^{[u]}$ be the tree defined in Step 5 of Algorithm 1. For any $v \in V\left(T_{1}\right)-\{u\}$ we compute the label $L_{T_{1}^{[v]}}(v)$ by simply deleting all components except the last seven components from $L_{T^{[v]}}(v)$.

From Theorem 4.12, we can compute $L_{T_{1}^{[u]}}(u)$ in Step 6. The road in $L_{T_{1}^{[u]}}(u)$ is calculated at that time.

Note that the cop number of $T_{1}^{[u]}$ is the largest key $k$ in $L_{T_{1}^{[u]}}(u)$. From the proof of Theorem 4.13 , we know by utilizing the road in $T_{1}^{[u]}$, we can use this cop number $k$ to clear the subtrees whose corresponding items have keys less than $k$, which results in the lists $L_{1}, \ldots, L_{d}, L_{d+1}$ created in Step 7 . If no key in $L_{1}, \ldots, L_{d}, L_{d+1}$ is contained in two different lists, from Theorem $4.13, L_{T^{[u]}}(u)$ consists of the items of $L_{1}, \ldots, L_{d}$ and $L_{T_{1}^{[u]}}(u)$, and thus, it is indeed the label of $u$ in the subtree $T^{[u]}$; otherwise, let $k^{*}$ be the largest key appears in at least two lists in $L_{1}, \ldots, L_{d}, L_{d+1}$, which is found in Step 9. Let $K=\left(k_{1}, \ldots, k_{\ell}\right)$ be a decreasing sequence created in Step 10, and let the corresponding items 
be $\left(Q_{1}, \ldots, Q_{\ell}\right)$, where $Q_{i}=\left(k_{i}, x_{i}, P_{i}\right), 1 \leq i \leq \ell$, is an item with key $k_{i}$ and attributes $x_{i}$ and $P_{i}$. Note that $k_{\ell}=k^{*}$. So either $u$ has at least two $k_{\ell^{-}}$ branching descendants or $u$ has one $k_{\ell}$-branching descendant and $\mathrm{c}_{1}\left(T_{1}^{[u]}\right)=k$. Similar to the proof of Theorem 4.14 and Theorem 4.13 , we have $\mathrm{c}_{1}\left(T^{[u]}-\right.$ $\left.\bigcup_{j=1}^{\ell-1} V\left(T^{\left[x_{j}\right]}\right)\right) \geq k_{\ell}+1$. If $k_{\ell-1}>k_{\ell}+1$, then in Step 11 we have $h=\ell$ and we update $K$ by $\left(k_{1}, \ldots, k_{\ell-1}, k_{\ell}^{\prime}\right)$, where $k_{\ell}^{\prime}=k_{\ell}+1$. If $k_{\ell-1}=k_{\ell}+1$, then we have $\mathrm{c}_{1}\left(T^{[u]}-\bigcup_{j=1}^{\ell-2} V\left(T^{\left[x_{j}\right]}\right)\right) \geq k_{\ell}+2$. Keep going in this way, we can find the smallest index $h$ in $K$, where $1 \leq h \leq \ell$, such that $k_{h}=k_{h+1}+1=\cdots=k_{\ell}+(\ell-h)$. Thus we can update $K$ by $\left(k_{1}, \ldots, k_{h-1}, k_{h}^{\prime}\right)$ where $k_{h}^{\prime}=k_{h}+1$ in Step 11 . Let $\left.T_{2}^{[u]}=T^{[u]}-\bigcup_{j=1}^{h-1} V\left(T^{\left[x_{j}\right]}\right)\right)$. As $T_{2}^{[u]}-N^{3}[u]$ does not have any components with cop number $k_{h}^{\prime}$ then $u$ is a hub in $T_{2}^{[u]}$. According to Definition 4.5 , $u$ is a road in $T_{2}^{[u]}$. From the structure of $T_{2}^{[u]}$, we have $x_{h}=\perp$, and $I_{\mathrm{wb}}^{k_{h}^{\prime}}(u)=J_{\mathrm{w}}^{k_{h}^{\prime}}(u)=$ $I_{\mathrm{pb}}^{k_{h}^{\prime}}(u)=J^{k_{h}^{\prime}}(u)=0$. From Lemma 4.9 we know each $T^{\left[x_{i}\right]}, 1 \leq i \leq h-1$ contains a road containing two utmost- $k$-pre-branching vertices. Therefore, the list $X$ generated in Step 11 is indeed the label of $u$ in the subtree $T^{[u]}$. We repeat Steps 411 until the root $u_{n}$ of the tree has a label $L_{T^{\left[u_{n}\right]}}\left(u_{n}\right)$.

In the remainder of this section, we analyze the running time of Algorithm 1.

Lemma 4.16. Suppose that each $S_{i}=\left(t_{m_{i}}^{i}, t_{m_{i}-1}^{i}, \ldots, t_{1}^{i}\right), 1 \leq i \leq d$, is a list of strictly decreasing positive integers. Let $s$ be the largest number in $S_{1}, \ldots, S_{d}$ which occurs in at least two lists if one exists; otherwise, let $s=0$. Then $s$ can be determined in $O\left(\max \left\{t_{m_{1}}^{1}, \ldots, t_{m_{d}}^{d}\right\}+d\right)$ time.

Proof. It takes $O(d)$ time to sort the first components $t_{m_{i}}^{i}, 1 \leq i \leq d$, in the 
lists in descending order using the bucket sort. W.l.o.g., suppose after sorting we have $t_{m_{1}}^{1} \geq t_{m_{2}}^{2} \geq \cdots \geq t_{m_{d}}^{d}$. Then $s$ can be determined by the following procedure.

Initially set $s \leftarrow 0$ and $S \leftarrow S_{1}$.

For $i:=2$ to $d$, do

For $t \in\left\{t_{m_{i}}^{i}, \ldots, t_{1}^{i}\right\} \cup\{s+1\}$ from $t_{m_{i}}^{i}$ down to $\max \left\{t_{1}^{i}, s+1\right\}$, do

If $t$ is found in $S$, then update $s \leftarrow t$ and

if $s=t_{m_{i}}^{i}$ or $i=d$, then return $s$, else, break the inner loop; else, insert $t$ into $S$.

In this procedure, we scan $S$ from the left (the largest number) to the right to find the target number $t$ or insert it into $S$. Since $S$ contains at most $t_{m_{1}}^{1}$ numbers, the number of insertions is at most $t_{m_{1}}^{1}$. So the runtime of the procedure is $O\left(t_{m_{1}}^{1}+d\right)$, which is $O\left(\max \left\{t_{m_{1}}^{1}, \ldots, t_{m_{d}}^{d}\right\}+d\right)$.

Lemma 4.17. Let $f(n)$ be a function defined on the positive integers by the recurrence equation

$$
f(n)= \begin{cases}c, & n=1 \\ f\left(n_{1}\right)+c, & d=1, n \geq 2, \\ \max _{M}\left\{\sum_{i=1}^{d} f\left(n_{i}\right)+c\left(\left\lceil\log n_{1}\right\rceil+d\right)\right\}, & d \geq 2, n \geq 3,\end{cases}
$$

where $M=\left\{\left(n_{1}, \ldots, n_{d}\right) \mid n_{1} \geq \cdots \geq n_{d} \geq 1\right.$, and $\left.\sum_{i=1}^{d} n_{i}=n-1\right\}$, and $c \geq 1$ is a constant. Then $f(n)$ is $O(n)$.

Lemma 4.17 can be proved similarly to Lemma 3.13 in 22. 
Theorem 4.18. Algorithm 1 can be implemented in linear time.

Proof. Let $T$ be a tree with $n \geq 3$ vertices. In Algorithm 11, Step 2 requires $O(n)$ time by the topological sort. In each iteration of the loop from Steps 4 to 11. we compute the label of $u$ in the subtree $T^{[u]}$. Let $v_{1}, \ldots, v_{d}$ be the children of $u$ whose labels have been calculated. When we calculate those labels, we use a linked list for each label. We also use a flag associated with every label to indicate whether the label contains $\perp$. So Step 4 requires $O(d)$ time to find $I_{\perp}$ and $I_{b}$.

In Step 5 , we construct the subtree $T_{1}^{[u]}$. In order to save time for scanning the labels, we compress the label representation. For a sub-list of items with consecutive keys in a label, we use an interval to represent them. For any $v \in$ $V\left(T_{1}\right)-\{u\}$ we compute the label $L_{T_{1}^{[v]}}(v)$ by simply deleting all components except the last seven components from $L_{T^{[v]}}(v)$. As we are using linked list to store the labels Step 5 requires $O(d)$ time.

In Step 6 we compute $L_{T_{1}^{[u]}}(u)$ from $L_{T_{1}^{\left[v_{1}\right]}}\left(v_{1}\right), \ldots L_{T_{1}^{\left[v_{d}\right]}}\left(v_{d}\right)$ by using Theorem 4.12. Its easy to see that all cases except Case (5.2) and Case (5.3) in Theorem 4.12 takes $O(d)$ time.

In (5.3) to find the utmost- $k$-pre-branching vertex in $T_{1}^{[u]}$ we check the sixth component of each label of $d$ children of $u$ and find the child $v_{1}$ with $I_{\mathrm{pb}}^{k}\left(v_{1}\right)=1$. Now we check the third component of $v_{1}$. If $P^{v_{1}}$ contains $v_{1}$ then $v_{1}$ itself is the utmost- $k$-pre-branching vertex in $T_{1}^{\left[v_{1}\right]}$. Then $u v_{1}$ is the road in $T_{1}^{[u]}$. If $P^{v_{1}}$ contains $v_{1}$ and the utmost- $k$-pre-branching vertex $z_{1}$ in $T_{1}^{\left[v_{1}\right]}$, then $u \sim z_{1}$ is the road. So to find the road we check the label of each $d$ children 
of $u$. Similarly we can find the utmost- $k$-pre-branching vertices in Case (5.2), So all cases in Theorem 4.12, take $O(d)$ time to compute the road in $L_{T_{1}^{[u]}}(u)$. So the complexity after executing Step 6 for each vertex is bounded by the number of incident edges $O(d)$.

In Step 7, it takes $O(d)$ time to modify the labels to obtain the lists $L_{1}, \ldots, L_{d+1}$. Let $t_{m_{i}}^{i}, 1 \leq i \leq d+1$, be the largest key in $L_{i}$. In Steps 8 and 9 , from Lemma 4.16 , it takes $O\left(\max \left\{t_{m_{1}}^{1}, \ldots, t_{m_{d+1}}^{d+1}\right\}+d\right)$ time to determine if all keys in $L_{1}, \ldots, L_{d+1}$ are different or find the largest repeated key $k^{*}$ in the lists. Using the compressed representation of labels, the runtime of Steps 10 and 11 is $O\left(\max \left\{t_{m_{1}}^{1}, \ldots, t_{m_{d+1}}^{d+1}\right\}+d\right)$.

From Lemma 4.17, the total runtime of the loop from Steps 4 to 11 is $O(n)$. Thus Algorithm 1 can be implemented in linear time.

\subsection{An Optimal Clearing Guide of a Tree}

$\operatorname{Algorithm} \operatorname{CLEAR}\left(T^{[u]}\right)$ is an optimal clearing guide of a tree $T^{[u]}$.

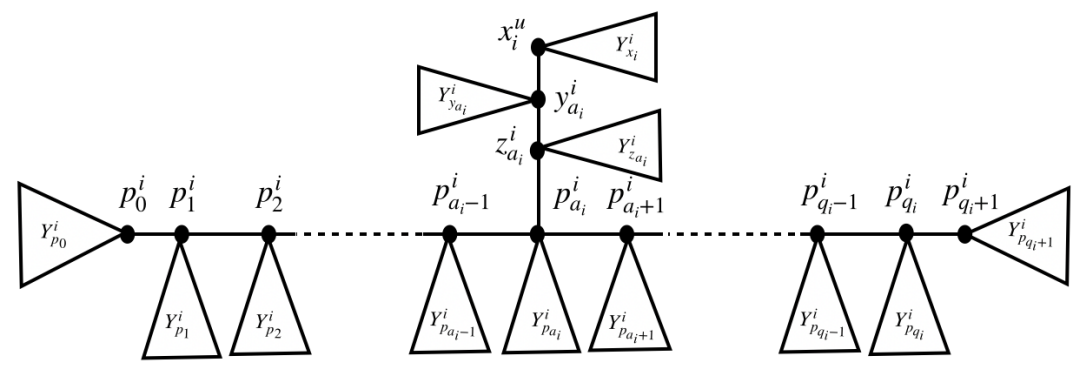

Figure 4.11: Tree $T_{i}^{\left[x_{i}^{u}\right]}, i \in\{2,3, \ldots, m\} !^{1}$

\footnotetext{
${ }^{1}$ In Figure $4.11, T_{i}^{\left[x_{i}^{u}\right]}$ is a subtree of $T_{i}^{[u]}$.
} 


\section{Algorithm 2 Clear $\left(T^{[u]}\right)$}

Input: $\mathrm{A}$ rooted tree $T^{[u]}$ with $n \geq 3$ vertices and the labels of all vertices in $T^{[u]}$. The label of root $u$ in $T^{[u]}$ is $L(u)=$ $\left(t_{m}^{u}, x_{m}^{u}, P_{m}^{u} ; \ldots ; t_{1}^{u}, x_{1}^{u}, P_{1}^{u} ; I_{\mathrm{wb}}^{t_{1}^{u}}(u), J_{\mathrm{w}}^{t_{1}^{u}}(u) ; I_{\mathrm{pb}}^{t_{1}^{u}}(u), J^{t_{1}^{u}}(u)\right)$.

Output: A guide to clear $T^{[u]}$ with $c_{1}\left(T^{[u]}\right)$ cops.

If the robber is seen by a cop at any moment then the robber will be captured by the cop in at most $b$ rounds, where $b$ is the diameter of the tree.

At the beginning of the game place all $c_{1}\left(T^{[u]}\right)$ cops on $u$. All cops are free at this moment. Assign grey color to all vertices of $T^{[u]}$ to indicate them as dirty.

if $m=1$ then,

\section{BASE-STRATEGy $\left(T^{[u]}\right)$.}

return

else

For $1 \leq i<m, T_{i}^{[u]}=T^{[u]}-\bigcup_{j=i+1}^{m} V\left(T^{\left[x_{j}^{u}\right]}\right)$ and $T_{m}^{[u]}=T^{[u]}$. For $2 \leq$ $i \leq m, P_{i}^{u}$ contains two terminal vertices $p_{0}^{i}$ and $p_{q_{i}+1}^{i}$ of a road in $T_{i}^{[u]}$. Let $A^{i}=\left\{p_{1}^{i}, \ldots, p_{q_{i}}^{i}\right\}$ be the set of internal road vertices. Suppose that $p_{a_{i}}^{i} \in A^{i}$ be the great grand child of $x_{i}^{u}$. Let $y_{a_{i}}^{i}$ and $z_{a_{i}}^{i}$ be two vertices on the path $x_{i}^{u} p_{a_{i}}^{i}$ where $z_{a_{i}}^{i}$ is the parent of $p_{a_{i}}^{i}$ and $y_{a_{i}}^{i}$ is the child of $x_{i}^{u}$. Let $w_{i}$ be the parent of $x_{i}^{u}$. Let $Y_{p_{0}}^{i}$ (resp. $Y_{p_{q_{i}+1}}^{i}$ ) be the component in $T_{i}^{[u]}-A^{i}$ which contains $p_{0}^{i}$ (resp. $\left.p_{q_{i}+1}^{i}\right)$. Let $Y_{p_{j}}^{i}, 1 \leq j \leq a_{i}-1$,(resp. $Y_{p_{j}}^{i}, a_{i}+1 \leq j \leq q_{i}$ ) be the component containing $p_{j}^{i}$ in the forest $T_{i}^{[u]}-\left\{p_{j-1}^{i} p_{j}^{i}, p_{j}^{i} p_{j+1}^{i}\right\}$. Let $Y_{x_{i}}^{i}$ (resp. $Y_{y_{a_{i}}}^{i}, Y_{z_{a_{i}}}^{i}$ and $Y_{p_{a_{i}}}^{i}$ ) be the component containing $x_{i}^{u}$ (resp. $y_{a_{i}}^{i}$, $z_{a_{i}}^{i}$ and $p_{a_{i}}^{i}$ ) in the forest $T_{i}^{[u]}-\left\{x_{i}^{u} y_{a_{i}}^{i}, x_{i}^{u} w_{i}\right\}$ (resp. $T_{i}^{[u]}-\left\{x_{i}^{u} y_{a_{i}}^{i}, y_{a_{i}}^{i} z_{a_{i}}^{i}\right\}$, $T_{i}^{[u]}-\left\{y_{a_{i}}^{i} z_{a_{i}}^{i}, z_{a_{i}}^{i} p_{a_{i}}^{i}\right\}$ and $T_{i}^{[u]}-\left\{p_{a_{i}}^{i} p_{a_{i}-1}^{i}, z_{a_{i}}^{i} p_{a_{i}}^{i}, p_{a_{i}}^{i} p_{a_{i}+1}^{i}\right\}$ ) (see Figure 4.11). for $i:=m$ to 2 do

Slide all $\mathrm{c}_{1}\left(T_{i}^{[u]}\right)=t_{i}^{u}$ free cops to $p_{0}^{i}$.

for $j:=0$ to $a_{i}-1$ do

$\operatorname{Hub-STRATEGy}\left(Y_{p_{j}}^{i}, p_{j}^{i}\right)$; slide all free $t_{i}^{u}$ cops from $p_{j}^{i}$ to $p_{j+1}^{i}$.

One cop vibrates between $z_{a_{i}}^{i}$ and $y_{a_{i}}^{i}$. Indicate the cop as busy.

Slide all $t_{1}^{u}$ free cops to $u$; then BASE-STRATEGy $\left(T_{1}^{[u]}\right)$.

for $i:=2$ to $m$ do

Slide all $t_{i}^{u}-1$ free cops to $x_{i}^{u}$; then Hub-Strategy $\left(Y_{x_{i}}^{i}, x_{i}^{u}\right)$

Slide all $t_{i}^{u}-1$ free cops to $y_{a_{i}}^{i}$; then Hub-STRATEGy $\left(Y_{y_{a_{i}}}^{i}, y_{a_{i}}^{i}\right)$. Indicate $t_{i}^{u}$-th cop as free.

Slide all $t_{i}^{u}$ free cops to $z_{a_{i}}^{i}$; then HuB-STRATEGy $\left(Y_{z_{a_{i}}}^{i}, z_{a_{i}}^{i}\right)$ 
Slide all $t_{i}^{u}$ free cops to $p_{a_{i}}^{i}$; then HuB-StRATEGY $\left(Y_{p_{a_{i}}}^{i}, p_{a_{i}}^{i}\right)$

Slide all $t_{i}^{u}$ free cops to $p_{a_{i}+1}^{i}$;

for $j:=a_{i}+1$ to $q_{i}$ do

$\operatorname{HuB}-\operatorname{StRATEGy}\left(Y_{p_{j}}^{i}, p_{j}^{i}\right)$; then slide all $t_{i}^{u}$ free cops from $p_{j}^{i}$ to $p_{j+1}^{i}$.

$\operatorname{HuB-STRATEGy}\left(Y_{p_{q_{i}+1}}^{i}, p_{q_{i}+1}^{i}\right)$

Slide all $t_{m}^{u}$ free cops back to $u$.

return

function Hub-STRATEGy $\left(T^{[v]}, v\right)$

Let $v_{1}, \ldots, v_{d}$ be all children of $v$. For each $v_{i}, 1 \leq i \leq d$, let $v_{i j}, 1 \leq j \leq$ $m_{i}$, be all children of $v_{i}$. For each $v_{i j}, 1 \leq j \leq m_{i}$, let $v_{i j p}, 1 \leq p \leq a_{i j}$, be all children of $v_{i j}$. For each $v_{i j p}, 1 \leq p \leq a_{i j}$, let $v_{i j p q}, 1 \leq q \leq b_{i j p}$, be all children of $v_{i j p}$.

For each $v_{i}, 1 \leq i \leq d$, for each $v_{i j}, 1 \leq j \leq m_{i}$, for each $v_{i j p}, 1 \leq p \leq a_{i j}$, for each $v_{i j p q}, 1 \leq q \leq b_{i j p}$, if $v$ (resp. $v_{i}$ ) does not have any child, then $v$ (resp. $v_{i}$ ) is cleared by the cop sitting at $v$, or if $v_{i j}$ (resp. $v_{i j p}$ ) does not have any child then sliding a cop from $v_{i}$ to each $v_{i j}$ clears the subtree rooted at $v_{i j}$, otherwise, let one cop $\lambda$ vibrates between $v_{i}$ and $v_{i j}$ and indicate the cop as busy, in the meantime, slide other free cops to $v_{i j p q}$ and $\operatorname{CLEAR}\left(T^{\left[v_{i j p q}\right]}\right)$ until the subtree rooted at $v_{i j}$ is cleared.

Indicate $\lambda$ as free.

Assign green color to grey vertices of $T^{[v]}$ to indicate $T^{[v]}$ as a cleared zone.

return

\section{function BASE-STRATEGY $\left(T^{[u]}\right)$}

\section{if $P_{1}^{u}=u$ then}

$\operatorname{Hub-STRATEGy}\left(T^{[u]}, u\right)$

else

$P_{1}^{u}$ contains two terminal vertices $p_{0}^{1} p_{q_{1}+1}^{1}$ of a road. Let $A^{1}=$ $\left\{p_{1}^{1}, \ldots, p_{q_{1}}^{1}\right\}$ be the set of internal road vertices. Let $Y_{p_{0}}^{1}\left(\right.$ resp. $\left.Y_{p_{q_{1}+1}}^{1}\right)$ be the component in $T^{[u]}-A^{1}$ containing $p_{0}^{1}$ (resp. $\left.p_{q_{1}+1}^{1}\right)$. Let $Y_{p_{j}}^{1}, 1 \leq j \leq q_{1}$, be the component containing $p_{j}^{1}$ in the forest $T^{[u]}-\left\{p_{j-1}^{1} p_{j}^{1}, p_{j}^{1} p_{j+1}^{1}\right\}$.

Slide all $t_{1}^{u}$ free cops to $p_{0}^{1}$.

for $j:=0$ to $q_{1}$ do

$\operatorname{HuB}-\operatorname{StRATEGY}\left(Y_{p_{j}}^{1}, p_{j}^{1}\right)$; then slide all $t_{1}^{u}$ free cops from $p_{j}^{1}$ to $p_{j+1}^{1}$.

$\operatorname{HuB-STRATEGY}\left(Y_{p_{q_{1}+1}}^{1}, p_{q_{1}+1}^{1}\right)$

Slide all $t_{1}^{u}$ free cops back to $u$.

return 
Lemma 4.19. Let $T^{[u]}$ be a tree. If the labels of all vertices are known, then we can construct a clearing guide for $T^{[u]}$ in $O(n \log n)$ time.

Proof. The correctness of Algorithm 2 follows from our discussion in Sections 4.1, 4.2 and 4.3. We now analyze the running time of Algorithm 2. At the beginning of the game placing $\mathrm{c}_{1}\left(T^{[u]}\right)=t$ cops on $u$ takes $O(t)$ time, setting the indicator of $t$ cops as free takes $O(t)$ time, and assigning grey color to all vertices of $T^{[u]}$ takes $O(n)$ time since $n$ is the number of vertices in $T^{[u]}$. To keep track of free cops and their associated location we maintain a queue. The step of sliding $t$ free cops to a vertex $v$ consists of $s$ rounds where $s$ is the distance of the farthest cop from $v$. Since input tree $T^{[u]}$ has $n$ vertices, the sliding actions of cops at each step take at most $O(n)$ time. The core function of Algorithm 2 is Hub-Strategy. We can implement Hub-Strategy using depth first search algorithm which takes $O(n)$, and assigning green color to the grey vertices at the end of Hub-Strategy also takes $O(n)$ time. Hence the complexity of implementing HuB-Strategy is $O(n)$. If $m>1$ tree $T^{[u]}$ is partitioned into $T_{1}^{[u]}, T_{2}^{[u]} \ldots, T_{m}^{[u]}$ trees. Each $T_{i}^{[u]}, 1<i \leq m$ contains an avenue $p_{1}^{i}, \ldots, p_{q}^{i}$. The $t_{i^{-}}$weakly-branching vertex $p_{a_{i}}^{i}$ is the great grand child of $x_{i}$ and is on the avenue. The clearing guide clears the subtree rooted at $p_{0}^{i}$ with $t_{i}$ cops by using Hub-StRATEGY, then all free $t_{i}$ cops slide from $p_{0}^{i}$ to $p_{1}^{i}$, then clear the subtree rooted at $p_{1}^{i}$ with $t_{i}$ cops by using HuB-STRATEGY, and keep doing same until $p_{a_{i}}^{i}$. Then the $t_{i}$-th cop keeps vibrating on the parent of $p_{a_{i}}^{i}$ and the child of $x_{i}$ until $T_{j}^{[u]}, 1 \leq j<i$ is cleared. This vibrating actions take $O(\log n)$ time. We set the indicator of the $t_{i}$-th cop as busy which takes $O(1)$ time. We do the above mentioned steps for each $T_{i}^{[u]}, m \geq i \geq 2$. To 
accomplish this we need $O(n \log n)$ time, since we divide the avenue of each $T_{i}^{[u]}$ into half and sliding actions and HuB-STRATEGY both require $O(n)$ time. Tree $T_{1}$ is cleared using BAse-Strategy which takes $O(n)$ time. Similarly clearing the rest of the dirty vertices in $T_{i}^{[u]}, m \geq i \geq 2$ take $O(n \log n)$ time. If $m=1$ then $T^{[u]}$ is cleared using BASE-Strategy which takes $O(n)$ time. Therefore, the total running time of Algorithm 2 is $O(n \log n)$.

Remark. The clearing guide in Algorithm 2 contains some sliding actions and by simply following those sliding actions of cops the cop player can clear an input tree $T^{[u]}$. A step of sliding $t$ free cops to a vertex $v$ is actually consists of s rounds, where free cops refer to those cops not vibrating on any edge and $s$ is the distance of farthest cop from $v$. Within $s$ rounds if some cops reach $v$ then those cops will sit on $v$ until the farthest cop reaches to $v$. So, in a round $t_{1} \leq t$ cops sit on $v, t-t_{1}$ cops move towards $v$ and the rest of the cops in $T^{[u]}$ keep vibrating. If the robber is seen by a cop at some moment, then according to proposition 2.1 the robber can be captured by this cop in a finite number of rounds. If the robber ever enters the cleared zone then the robber will be seen by a cop in the next round and captured by the cop in a finite number of rounds, otherwise the cleared zone will be kept cleared until the end of the game.

Lemma 4.20. Let $T^{[u]}$ be a tree. In an optimal cop-win strategy computed by Algorithm 2 let $\alpha$ be the maximum number of vibrating cops in a round. Then $\mathrm{c}_{1}\left(T^{[u]}\right)=\alpha+1$.

Proof. Let $L(u)=\left(t_{m}^{u}, x_{m}^{u}, P_{m}^{u} ; \ldots ; t_{1}^{u}, x_{1}^{u}, P_{1}^{u} ; I_{\mathrm{wb}}^{t_{1}^{u}}(u), J_{\mathrm{w}}^{t_{1}^{u}}(u) ; I_{\mathrm{pb}}^{t_{1}^{u}}(u), J^{t_{1}^{u}}(u)\right)$ and 
$\mathrm{c}_{1}\left(T^{[u]}\right)=t_{m}^{u}=k>1$. If $m>1$, Algorithm 2 calls Hub-Strategr $\left(T^{[v]}, v\right)$ to clear a subtree of $T^{[u]}$ rooted at a pre-avenue vertex $v$. If $m=1$, then if $P_{m}^{u}=u$, Algorithm 2 calls Hub-Strategy $\left(T^{[u]}, u\right)$, otherwise Algorithm 2 calls Hub-Strategy $\left(T^{[v]}, v\right)$ to clear a subtree of $T^{[u]}$ rooted at a pre-avenue vertex or a street vertex $v$. As $u$ (resp. $v$ ) is a hub in $T^{[u]}$ (resp. $T^{[v]}$ ) and $\mathrm{c}_{1}\left(T^{[u]}\right)=k\left(\right.$ resp. $\left.\mathrm{c}_{1}\left(T^{[v]}\right)=k\right)$, then $T^{[u]}-N^{3}[u]\left(\operatorname{resp} . T^{[v]}-N^{3}[v]\right)$ has at least three components $H_{1}, H_{2}, H_{3}$ with cop number $k-1$ such that the path between any two of them contains $u$ (resp. $v$ ). Algorithm 2 keeps one cop vibrating on a child say $u_{1}$ (resp. $v_{1}$ ) and a grandchild say $u_{2}$ (resp. $v_{2}$ ) of $u$ (resp. $v$ ) and calls itself to clear $H_{i}, i \in\{1,2,3\}$. The vibrating cop keeps vibrating until the subtree rooted at $u_{2}$ (resp. $v_{2}$ ) is cleared. As Algorithm 2 calls itself to clear $H_{i}, i \in\{1,2,3\}$, if the road in $H_{i}$ is equal to the root of $H_{i}$ say $q_{i}$ then Hub-Strategr $\left(H_{i}^{\left[q_{i}\right]}, q_{i}\right)$ is called to clear $H_{i}$, otherwise Hub$\operatorname{StRategy}\left(H_{i}^{\left[p_{i}\right]}, p_{i}\right)$ is called to clear a subtree of $H_{i}$ rooted at a pre-avenue vertex or a street vertex $p_{i}$. As $q_{i}\left(\right.$ resp. $\left.p_{i}\right)$ is a hub in $H_{i}^{\left[q_{i}\right]}\left(\right.$ resp. $\left.H_{i}^{\left[p_{i}\right]}\right)$ then $H_{i}^{\left[q_{i}\right]}-N^{3}\left[q_{i}\right]$ (resp. $\left.H_{i}^{\left[p_{i}\right]}-N^{3}\left[p_{i}\right]\right)$ has at least three components $S_{1}, S_{2}, S_{3}$ with cop number $k-2$ such that the path between any two of them contains $q_{i}\left(\right.$ resp. $\left.p_{i}\right)$. If $\mathrm{c}_{1}\left(H_{i}^{\left[q_{i}\right]}\right)>1\left(\right.$ resp. $\left.\mathrm{c}_{1}\left(H_{i}^{\left[p_{i}\right]}\right)>1\right)$, Algorithm 2 keeps one cop vibrating on a child say $q_{i}^{1}$ (resp. $p_{i}^{1}$ ) and a grandchild say $q_{i}^{2}$ (resp. $p_{i}^{2}$ ) of $q_{i}$ (resp. $p_{i}$ ) and calls itself to clear $S_{i}, i \in\{1,2,3\}$. The vibrating cop keeps vibrating until the subtree rooted at $q_{i}^{2}$ (resp. $p_{i}^{2}$ ) is cleared. So now in a round there are two vibrating cops and $k-2$ cops move to clear $S_{i}, i \in\{1,2,3\}$. As Algorithm 2 calls itself to clear $S_{i}$ the previous mentioned steps are followed for $S_{i}, i \in\{1,2,3\}$. So there is a round when three cops are vibrating and 
$k-3$ cops move to clear a subtree of $S_{i}, i \in\{1,2,3\}$ with cop number $k-3$. From the above discussion we can see there is a round when $k-1$ cops are vibrating and one cop moves to clear a subtree with cop number one. Let $W$ be the subtree with cop number one. If the road in $W$ is equal to the root of $W$ say $w$ then Hub-Strategy $\left(W^{[w]}, w\right)$ is called to clear $W$, otherwise Hub$\operatorname{Strategr}\left(W^{[y]}, y\right)$ is called to clear a subtree of $W$ rooted at a pre-avenue vertex or a street vertex $y$. As $w$ (resp. $y$ ) is a hub in $W^{[w]}\left(\right.$ resp. $\left.W^{[y]}\right)$ and $\mathrm{c}_{1}\left(W^{[w]}\right)=1\left(\operatorname{resp} . \mathrm{c}_{1}\left(W^{[y]}\right)=1\right)$ then $W^{[w]}-N^{3}[w]\left(\right.$ resp. $\left.W^{[y]}-N^{3}[y]\right)$ is empty. If $w$ (resp. $y$ ) does not have any child or any grandchild then the cop will sit on $w$ (resp. $y$ ) and the subtree is cleared, otherwise, let $w_{i}$ (resp. $y_{i}$ ), $1 \leq i \leq d$ be the children and $w_{i j}$ (resp. $y_{i j}$ ), $1 \leq j \leq m_{d}$, be the grandchildren of $w$ (resp. $y$ ) and sliding the cop from each $w_{i}$ (resp. $y_{i}$ ) to each $w_{i j}$ (resp. $y_{i j}$ ) will clear the subtree $W^{[w]}$ (resp. $W^{[y]}$ ). So the cop does not need to vibrate between two vertices for more than two consecutive rounds. Then there is no vibrating cop in that subtree. So according to Algorithm 2 in this case the number of vibrating cops in a round in $T^{[u]}$ is $k-1$. If the cop does not follow Algorithm 2 then the cop can vibrate between $w_{i}$ (resp. $y_{i}$ ) and $w_{i j}$ (resp. $y_{i j}$ ) for four consecutive rounds and clear $W^{[w]}$ (resp. $W^{[y]}$ ). In that case the number of vibrating cops in a round in $T^{[u]}$ is $k$. 


\section{Chapter 5}

\section{Relations between $\mathrm{c}_{1}(T)$ and $\mathrm{c}_{0}(T)$}

In this Chapter, we explore relations between zero- and one-visibility cop numbers on trees. We divide our results into two sections here. In the first section, we give a relation between $c_{1}(T)$ and $c_{0}(T)$ for a general tree $T$. The second section contains relations between zero-visibility cop number and one-visibility cop number of some special trees.

\subsection{Comparing $c_{1}(T)$ and $c_{0}(T)$}

The following Theorem proves a relation between $\mathrm{c}_{1}(T)$ and $\mathrm{c}_{0}(T)$ for a general tree $T$.

Theorem 5.1. For any tree $T, \mathrm{c}_{1}(T) \leq \mathrm{c}_{0}(T) \leq 2 \mathrm{c}_{1}(T)$.

Proof. The first inequality is trivial. We only consider the second one. Let $T$ be a tree with $\mathrm{c}_{1}(T)=k$. Let $S_{1}$ be a 1 -visibility cops' strategy to clear 
$T$ with $k$ cops. We will describe a 0 -visibility cops' strategy $S_{0}$ that clears $T$ with $2 k$ cops.

For each cop $\lambda_{i}, 1 \leq i \leq k$, in the 1-visibility game, there are two corresponding cops $\lambda_{i}^{1}$ and $\lambda_{i}^{2}$ in the 0 -visibility game. From Theorem 3.7 and Corollary 3.8, T must contain a hub, an avenue, or a street. Suppose that $T$ contains a hub $u$ and $u$ is the root of $T$. Let $v_{i}, 1 \leq i \leq d$, be the children of $u$. For $1 \leq i \leq d$, let $w_{i j}$ be the children of $v_{i}, 1 \leq j \leq m_{i}$. Let $X_{i j}=\left\{x \in V\left(T^{[u]}\right) \mid \operatorname{dist}\left(x, v_{i}\right)=3\right.$ and $\left.\operatorname{dist}\left(x, w_{i j}\right)=2\right\}, 1 \leq i \leq d$, $1 \leq j \leq m_{i}$. In $S_{1}$, one cop, say $\lambda_{k}$, basically vibrates between $v_{i}$ and $w_{i j}$ while the other $k-1$ cops clear each subtree $T^{[x]}, x \in X_{i j}$, individually. Then in the 0 -visibility strategy $S_{0}, \lambda_{k}^{1}$ vibrates between $u$ and $v_{i}$ and $\lambda_{k}^{2}$ vibrates between $w_{i j}$ and its child between $w_{i j}$ and $T^{[x]}$ (the case would be trivial if $v_{i}$ or $w_{i j}$ do not have children), while the other $2(k-1)$ cops clear each subtree $T^{[x]}, x \in X_{i j}$, one by one. Thus, if $T$ contains a hub, then $S_{0}$ clears $T$ using $2 k$ cops. Similarly, we can show that $2 k$ cops can clear $T$ if it contains an avenue or a street. Therefore $\mathrm{c}_{0}(T) \leq 2 k$.

A tree is called a caterpillar if removing all degree-one vertices produces a path or an empty graph. The next lemma shows that the lower bound in Theorem 5.1 is tight.

Lemma 5.2. If $T$ is a caterpillar, then $\mathrm{c}_{0}(T)=\mathrm{c}_{1}(T)=1$.

In Figure 2.1, we see that $c_{1}(T)=3$ and $c_{1}\left(T^{\prime}\right)=2$. This means that adding a pendant edge to a tree may cause the cop number to increase by 
one. The next result gives a case where the cop number will not increase after adding a pendant edge.

Lemma 5.3. Let $T$ be a tree which has a path $v_{1} v_{2} v_{3} v_{4} u$ with $\operatorname{deg}_{T}\left(v_{1}\right)=1$ and $\operatorname{deg}_{T}\left(v_{i}\right)=2,2 \leq i \leq 4$. Let $H$ be a tree obtained from $T$ by adding a pendant edge $v_{0} v_{1}$ to the vertex $v_{1}$. Then $\mathrm{c}_{1}(H)=\mathrm{c}_{1}(T)$.

Proof. Pick $u$ as the root in both $T$ and $H$. From Algorithm 1 , we know that $v_{4}$ has the same label in both $T$ and $H$. Thus $\mathrm{c}_{1}(H)=\mathrm{c}_{1}(T)$.

From the above lemma, we can show that the lower bound in Theorem 5.1 is tight if for any two non-degree-2 vertices the distance between them is at least 8 .

Lemma 5.4. Let $T$ be a tree such that the distance between any pair of nondegree-2 vertices is at least 8. Then $\mathrm{c}_{0}(T)=\mathrm{c}_{1}(T)$.

Proof. Let $T$ be a tree such that the distance between any two non-degree-2 vertices is at least 8 . We first consider the case where $c_{1}(T)=1$. If $T$ contains a vertex $u$ with $\operatorname{deg}_{T}(u) \geq 3$, then the forest $T-N_{T}^{3}[u]$ contains at least three components with cop number at least one. It follows from Theorem 3.5 that $\mathrm{c}_{1}(T) \geq 2$. This is a contradiction. So $T$ is a path, which implies that $\mathrm{c}_{0}(T)=\mathrm{c}_{1}(T)=1$. Assume that if $\mathrm{c}_{1}(T) \leq k$, then $\mathrm{c}_{0}(T)=\mathrm{c}_{1}(T)$.

We now consider the case where $c_{1}(T)=k+1, k \geq 1$. Since $c_{1}(T) \geq 2$, $T$ must contain a vertex of degree at least 3. From Theorem 3.7 and the subsequent results in Section 3.2, $T$ must contain a hub, an avenue, or a street. Suppose that $T$ contains a hub, say $v$. If $\operatorname{deg}_{T}(v)=2$, let $u$ be a 
non-degree- 2 vertex that is nearest to $v$. It follows from Lemma 5.3 that $u$ is also a hub. So we can suppose $v$ is a hub of $T$ such that $\operatorname{deg}_{T}(v)>2$. In $T-N_{T}^{3}[v]$, the 1-visibility cop number of every component is at most $k$. Let $H$ be a component in $T-N_{T}^{3}[v]$ and let $v v_{1} v_{2} \cdots v_{8}$ be a path in $T$ such that $v_{4}$ is a leaf in $H$ and $\operatorname{deg}_{T}\left(v_{i}\right)=2,1 \leq i \leq 7$. Let $H^{\prime}$ be a tree obtained from $H$ by adding edges $v_{4} v_{3}, v_{3} v_{2}$ and $v_{2} v_{1}$. It follows from Lemma 5.3 that $\mathrm{c}_{1}\left(H^{\prime}\right)=\mathrm{c}_{1}(H) \leq k$. By the assumption, we have $\mathrm{c}_{0}\left(H^{\prime}\right)=\mathrm{c}_{1}\left(H^{\prime}\right) \leq k$. Thus, in $T-v$, the 0 -visibility cop number of each component is at most $k$. So in the 0 -visibility game, we can clear $T$ by stationing one cop on $v$ and using $k$ cops to clear each component of $T-v$. Hence $\mathrm{c}_{0}(T) \leq k+1$. Since for any tree its 0 -visibility cop number is always greater than or equal to its 1visibility cop number, we have $\mathrm{c}_{0}(T)=\mathrm{c}_{1}(T)=k+1$. If $T$ contains an avenue or a street, since Avenue-Strategy and Street-Strategy are based on Hub-Strategy, it is not hard to see that $\mathrm{c}_{0}(T)=\mathrm{c}_{1}(T)=k+1$.

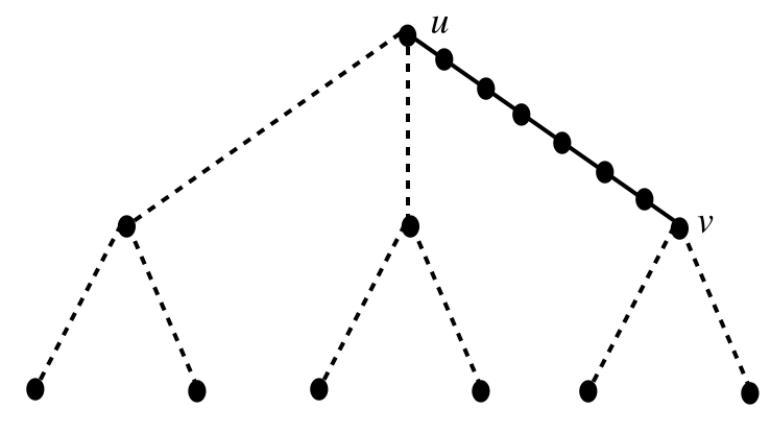

Figure 5.1: A tree where each dashed line represents a path of length at least 8.

The distance condition in Lemma 5.4 can be tight. For example, consider the tree $T$ in Figure 5.1, where every path between a pair of non-degree- 
2 vertices is of length at least 8 except the path between $u$ and $v$. Since $\operatorname{dist}(u, v)=7$, it is easy to see that $\mathrm{c}_{0}(T)=3$ and $\mathrm{c}_{1}(T)=2$.

The following lemma shows that the upper bound in Theorem 5.1 is also tight.

Lemma 5.5. Let $T$ be a caterpillar with at least one vertex of degree 3. Let $H$ be a tree obtained from $T$ by replacing every edge by a path of length 3. Then $\mathrm{c}_{0}(H)=2 \mathrm{c}_{1}(H)=2$.

\section{$5.2 \quad$ Special Trees}

This section focuses on some special trees. We show relations between zerovisibility cop number and one-visibility cop number of perfect binary trees and perfect $k$-ary trees.

In a rooted tree, the height of a vertex is the distance from the root to this vertex. The height of a rooted tree is the largest distance from the root to a leaf.

\subsubsection{Perfect Binary Trees}

A perfect binary tree $T^{2}$ is a rooted tree in which every internal vertex has exactly two children and all leaves have the same height. Here we calculate both one-visibility cop number and zero-visibility cop number for a perfect binary tree $T$ as a function of the height of the tree. We use $T_{h}^{2}$ to denote a 
perfect binary tree with height $h$.

Theorem 5.6. Let $T_{h}^{2}$ be a perfect binary tree with $h \geq 0$. Then $\mathrm{c}_{1}\left(T_{h}^{2}\right)=$ $\left\lceil\frac{h+1}{5}\right\rceil$ and $\mathrm{c}_{0}\left(T_{h}^{2}\right)=\left\lceil\frac{h+1}{3}\right\rceil$.

Proof. We first show $\mathrm{c}_{1}\left(T_{h}^{2}\right)=\left\lceil\frac{h+1}{5}\right\rceil$ using induction. If $0 \leq h \leq 4$, it is easy to see that one cop can clear the tree, and so the claim holds. Assume that the claim is true when $5 m \leq h \leq 5 m+4, m \geq 0$. We only need to show that the claim is also true when $5 m+5 \leq h \leq 5 m+9$.

Consider the case where $h=5 m+5$. Let $v$ be a child of the root $u$ in $T_{5 m+5}^{2}$. In the forest $T_{5 m+5}^{2}-N^{3}[v]$, there are at least two components which are isomorphic to $T_{5 m}^{2}$ and there is at least one component which is isomorphic to $T_{5 m+2}^{2}$ such that the path between any pair of the three components contains $v$ (see figure 5.2). From the assumption and Theorem 3.5. we have $\mathrm{c}_{1}\left(T_{5 m+5}^{2}\right) \geq$ $m+2$. Note that we can clear $T_{5 m+5}^{2}$ with $m+2$ cops by utilizing HubStRategy where $v$ is the hub. Thus $c_{1}\left(T_{5 m+5}^{2}\right)=m+2$.

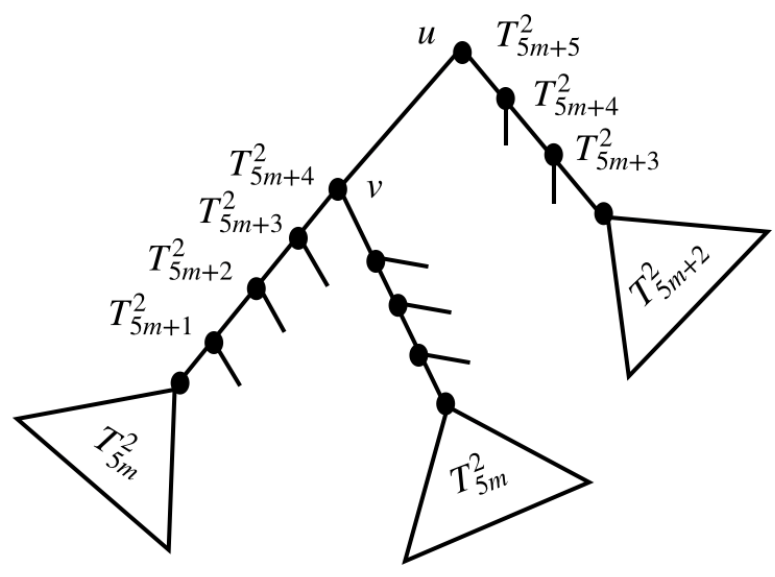

Figure 5.2: Tree $T_{5 m+5}^{2}$ with root $u$. 
Let $u$ be the root of $T_{5 m+9}^{2}$. In the forest $T_{5 m+9}^{2}-N^{3}[u]$, there are two components which are isomorphic to $T_{5 m+5}^{2}$ such that the path between them contains $u$ (see figure 5.3 p. So $u$ is an avenue vertex and $T_{5 m+9}^{2}$ can be cleared with $m+2$ cops by utilizing Avenue-Strategy.

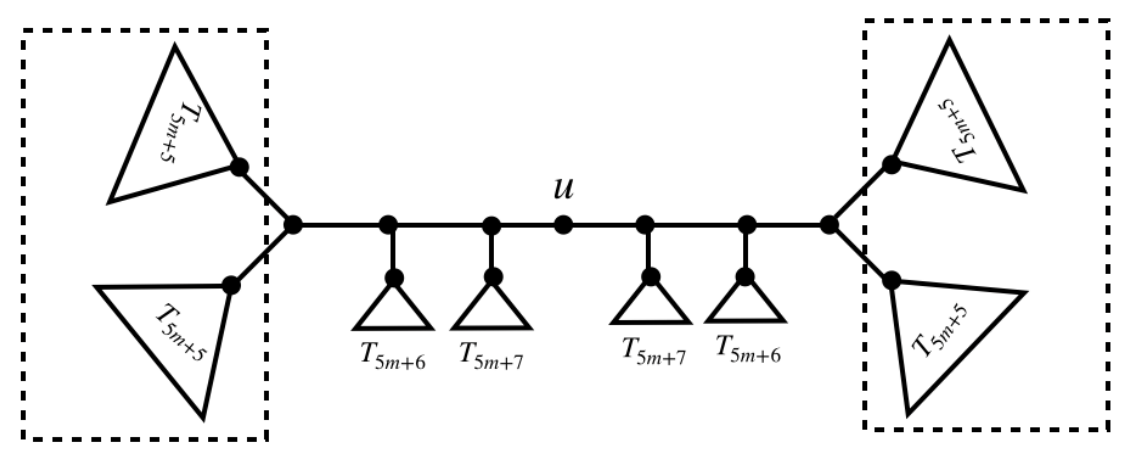

Figure 5.3: Tree $T_{5 m+9}^{2}$ with root $u$.

Therefore, $\mathrm{c}_{1}\left(T_{h}^{2}\right)=\left\lceil\frac{h+1}{5}\right\rceil$ for any $h \geq 0$.

Similarly to the above, we can show $\mathrm{c}_{0}\left(T_{h}^{2}\right)=\left\lceil\frac{h+1}{3}\right\rceil$ by Theorem 3.6 in $\lceil 7$.

\subsubsection{Perfect $k$-ary Trees}

A perfect $k$-ary tree is a rooted tree in which every internal vertex has $k$ children and all leaves have the same height. We use $T_{h}^{k}$ to denote a perfect $k$-ary tree with height $h$. Notice that the 1-visibility cop number of a perfect $k$-ary tree can be computed by Algorithm 1 .

Theorem 5.7. Let $T_{h}^{k}$ be a perfect $k$-ary tree with $k \geq 3$ and $h \geq 0$. Then $\mathrm{c}_{1}\left(T_{h}^{k}\right)=\left\lceil\frac{h+1}{4}\right\rceil$, and $\mathrm{c}_{0}\left(T_{h}^{k}\right)=\left\lceil\frac{h+1}{2}\right\rceil$. 
Proof. We use induction to show $\mathrm{c}_{1}\left(T_{h}^{k}\right)=\left\lceil\frac{h+1}{4}\right\rceil$. Observe that when $0 \leq h \leq$ 3, one cop can clear the tree, and thus, the claim holds. Assume that the claim is true when $4 m-4 \leq h \leq 4 m-1, m \geq 1$. We will show that the claim is also true when $4 m \leq h \leq 4 m+3$.

First consider the case where $h=4 m$. Let $u$ be the root of $T_{4 m}^{k}$. In the forest $T_{4 m}^{k}-N^{3}[u]$, there are at least three components which are isomorphic to $T_{4 m-4}^{k}$ such that the path between any pair of the three components contains $u$ (see Figure 5.4). From the assumption and Theorem 3.5, we have $c_{1}\left(T_{4 m}^{k}\right) \geq$ $m+1$. Since we can clear $T_{4 m}^{k}$ with $m+1$ cops by the Hub-Strategy where $u$ is the hub. Thus $c_{1}\left(T_{4 m}^{k}\right)=m+1$. Similarly, we can show that $\mathrm{c}_{1}\left(T_{4 m+i}^{k}\right)=m+1$ for $1 \leq i \leq 3$. Hence $\mathrm{c}_{1}\left(T_{h}^{k}\right)=\left\lceil\frac{h+1}{4}\right\rceil$ for any $T_{h}^{k}$ with $k \geq 3$ and $h \geq 0$.

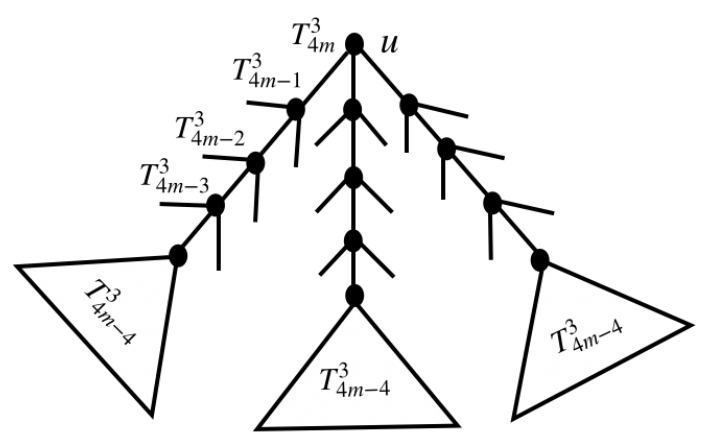

Figure 5.4: Tree $T_{4 m}^{3}$ with root $u$.

Similarly, from Theorem 3.6 in [7], we can show that $\mathrm{c}_{0}\left(T_{h}^{k}\right)=\left\lceil\frac{h+1}{2}\right\rceil$. 


\section{Chapter 6}

\section{Conclusions and Future Work}

\subsection{Summary}

In the original cops and robber game, the location of the robber is known throughout the game. In the zero-visibility cops and robber game, cops can only see the robber when both cops and robber occupy the same vertex. In this thesis, we explore another extreme, one-visibility cops and robber game where cops can see the robber when the distance between them is at most 1 . Here we have considered one-visibility cops and robber game only on trees. We have described an essential theorem for the lower bound of cop number on trees. Here we give structure properties in terms of the hub, avenue and street and also provide strategies to clear trees according to their structures. Throughout the thesis, we have considered only one robber in trees. However if we extend the game with more than one robber the strategy provided here 
can still clear trees. We have developed a linear algorithm for calculating the labels of a tree. Our focus was to compute the cop number of a tree and a road to clear the tree optimally. We have provided an optimal clearing guide to clear a tree. Moreover, we have established some relations between zero-visibility cop number and one-visibility cop number of a tree.

\subsection{Future Work}

There remains a considerable amount of future work concerning the onevisibility cops and robber game. We have considered only trees in this thesis, however, playing the one-visibility game on other graphs is yet to be explored. Finding efficient algorithms for computing the cop number of graphs like unicyclic graphs, CDGs, outerplanar graphs continue to be a challenge. Having optimal clearing strategies for 1-visibility cops and robber game on trees, its natural to consider other special classes of graphs such as planar graphs, cycledisjoint graphs. Moreover, it would be very interesting to construct relationships between more general $l$-visibility games. 


\section{References}

[1] D. Bienstock and P. Seymour. "Monotonicity in graph searching". Journal of Algorithms, 12(2): 239-245, 1991.

[2] A. Bonato and R.J. Nowakowski. "The game of cops and robbers on graphs". American Mathematical Society, Providence, Rhode Island, 2011.

[3] A. Bonato and B. Yang. "Graph searching and related problems". Handbook of Combinatorial Optimization, 2nd Edition, Panos Pardalos, DingZhu Du, and Ronald Graham (Eds.), pp.1511-1558, 2013, Springer New York.

[4] N. E. Clarke, D. Cox, C. Duffy, D. Dyer, S. L. Fitzpatrick, and M.-E. Messinger. "Limited visibility cops and robber". Discrete Applied Mathematics, 282: 53-64, 2020.

[5] N. E. Clarke and G. MacGillivray. "Characterizations of k-copwin graphs". Discrete Mathematics, 312(8): 1421-1425, 2012.

[6] D. Dereniowski, D. Dyer, R. M. Tifenbach, and B. Yang. "Zero-visibility cops and robber game on a graph". In: Fellows M., Tan X., Zhu B. (eds) 
Frontiers in Algorithmics and Algorithmic Aspects in Information and Management, Lecture Notes in Computer Science 7924, pages 175-186, 2013, Springer, Berlin, Heidelberg.

[7] D. Dereniowski, D. Dyer, R. Tifenbach, and B. Yang. "The complexity of zero-visibility cops and robber". Theoretical Computer Science, 607: 135-148, 2015.

[8] D. Dereniowski, D. Dyer, R. Tifenbach, and B. Yang. "Zero-visibility cops and robber and the pathwidth of a graph". Journal of Combinatorial Optimization, 29(3): 541-564, 2015.

[9] D. Dyer, B. Yang, and Ö. Yaşar. "On the fast searching problem". In: Fleischer R., Xu J. (eds) Algorithmic Aspects in Information and Management, Lecture Notes in Computer Science 5034, pages 143-154, AAIM 2008, Springer, Berlin, Heidelberg.

[10] F. V. Fomin, Petr A Golovach, Alexander Hall, Matúš Mihalák, Elias Vicari, and Peter Widmayer. "How to guard a graph?" In: Hong SH., Nagamochi H., Fukunaga T. (eds) Algorithms and Computation, Lecture Notes in Computer Science 5369, pages 318-329, ISAAC 2008, Springer, Berlin, Heidelberg.

[11] L. M. Kirousis and C. H. Papadimitriou. "Searching and pebbling". Theoretical Computer Science, 47: 205-218, 1986.

[12] N. Megiddo, S. L. Hakimi, M. R. Garey, D. S. Johnson, and C. H. Papadimitriou. "The complexity of searching a graph". Journal of the ACM 35(1): 18-44, 1988. 
[13] R.J. Nowakowski and P. Winkler. "Vertex-to-vertex pursuit in a graph". Discrete Mathematics, 43(2-3): 235-239, 1983.

[14] T. D. Parsons. "Pursuit-evasion in a graph". In: Alavi Y., Lick D.R. (eds) Theory and Applications of Graphs, Lecture Notes in Mathematics 642, pages 426-441, 1978, Springer, Berlin, Heidelberg.

[15] A. Quilliot. "Jeux et pointes fixes sur les graphes". Thèse de 3ème cycle, Université de Paris VI, 131-145, 1978.

[16] A. Tang. "Cops and robber with bounded visibility." Master's thesis, Dalhousie University, 2004.

[17] R. Tošić. "Vertex-to-vertex search in a graph". In Proceedings of the Sixth Yugoslav Seminar on Graph Theory, pp. 233-237, 1985.

[18] Y. Xue, B. Yang, F. Zhong, and S. Zilles. "A partition approach to lower bounds for zero-visibility cops and robber". In: Colbourn C., Grossi R., Pisanti N. (eds) Combinatorial Algorithms, Lecture Notes in Computer Science 11638, pages 442-454, IWOCA 2019, Springer, Cham.

[19] Y. Xue, B. Yang, and S. Zilles. "New results on the zero-visibility cops and robber game". In: Du DZ., Li L., Sun X., Zhang J. (eds) Algorithmic Aspects in Information and Management, Lecture Notes in Computer Science 11640, pages 316-328, AAIM 2019, Springer, Cham.

[20] B. Yang. "Fast edge searching and fast searching on graphs". Theoretical Computer Science, 412(12-14): 1208-1219, 2011. 
[21] B. Yang and T. Akter. "Computing the One-Visibility Copnumber of Trees". In: Zhang Z., Li W., Du DZ. (eds) Algorithmic Aspects in Information and Management, Lecture Notes in Computer Science 12290, pages 411-423, AAIM 2020, Springer, Cham.

[22] B. Yang, R. Zhang, Y. Cao, and F. Zhong. "Search Numbers in Networks with Special Topologies". Journal of Interconnection Networks, 19(01): $1-34,2019$.

[23] F. Yang. "1-visibility cops and robber problem". Honour's thesis, University of Prince Edward Island, 2012. 